DOE/EIA-0535(96)

Distribution Category UC-950

\title{
Fuel Oil and Kerosene Sales 1996
}

\section{August 1997}

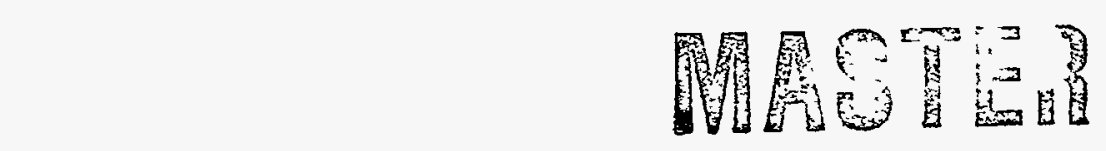

\section{Energy Information Administration}

Office of Oil and Gas

U.S. Department of Energy

Washington DC 20585 


\section{Contacts}

The Fuel Oil and Kerosene Sales 1996 report is prepared by the Energy Information Administration (EIA) under the general direction of Michael Griffey (202) 586-9601, Petroleum Marketing Division, Office of Oil and Gas.

Detailed, technical questions about tables referencing the form EIA-821 may be directed to:

Alice Lippert (202) 586-9600

\section{Fuel Oil and Kerosene Data Available Through Electronic Access}

Annual petroleum marketing data are available through electronic access that contains statistics from the $F u e l$ Oil and Kerosene Sales 1996 report. Included are annual sales data on petroleum volumes of kerosene, distillate fuel oils, and residual fuel oils by State. Annual historical data at the national level are provided in summary tables.

For Fuel Oil and Kerosene Sales on the Internet, access EIA's home page at http://www.eia.doe.gov.

For obtaining Fuel Oil and Kerosene Sales personal computer diskette by mail, order from:

Office of Scientific and Technical Information

Request Services

P.O. Box 62

Oak Ridge, Tennessee 37831

(615) 576-8401

National Technical Information Service (NTIS)

Office of Data Base Services

U.S. Department of Commerce

5285 Port Royal Road

Springfield, Virginia 22161

(703) $487-4650$

The Fuel Oil and Kerosene Sales diskette is in ASCII text format on a double-sided high-density 3.5 inch diskette for use with IBM-compatible personal computers.

Internet Addresses:

E-Mail: infoctr@eia.doe.gov

World Wide Web Site: http:/www.eia.doe.gov

Gopher Site: gopher://gopher.eia.doe.gov

FTP Site: ftp://ftp.eia.doe.gov

Further information as to content may be obtained from the National Energy Information Center (NEIC), telephone (202) 586-8800. 


\section{DISCLAIMER}

This report was prepared as an account of work sponsored by an agency of the United States Government. Neither the United States Government nor any agency thereof, nor any of their employees. make any warranty, express or implied, or assumes any legal liability or responsibility for the accuracy, completeness, or usefulness of any information, apparatus, product. or process disciosed, or represents that its use would not infringe privately owned rights. Reference herein to any specific commercial product, process. or service by trade name trademark, manufacturer, or otherwise does not necessarily constitute or imply its endorsement, recommendation, or favoring by the United States Government or any agency thereof. The views and opinions of authors expressed herein do not necessarily state or reflect those of the United States Government or any agency thereof. 


\section{DISCLAMMER}

Portions of this document may be illegible in electronic image products. Images are produced from the best available original document. 


\section{Contents}

\section{Page}

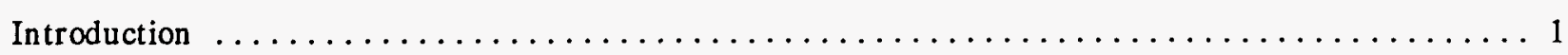

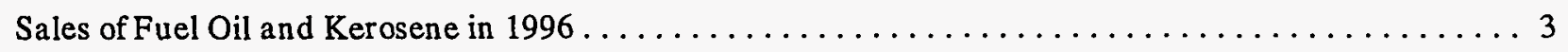

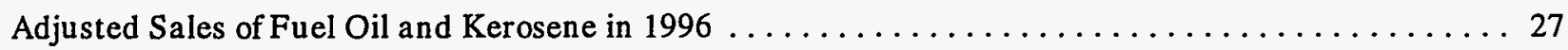

Appendix A: Technical Notes. ................................. 45

Appendix B: Form EIA-821: Annual Fuel Oil and Kerosene Sales Report $\ldots \ldots \ldots \ldots \ldots \ldots \ldots$ 


\section{Tables}

\section{Sales of Fuel Oil and Kerosene in 1996}

1. Sales of Distillate Fuel Oil by End Use in the United States: $1992-1996 \ldots \ldots \ldots \ldots \ldots \ldots 11$

2. Sales of Residual Fuel Oil by End Use in the United States: $1992-1996$. . . . . . . . . . 12

3. Sales of Kerosene by End Use in the United States: $1992-1996$. . . . . . . . . . . . . . . 12

4. Sales of Distillate Fuel Oil by End Use, 1995 and $1996 \ldots \ldots \ldots \ldots \ldots \ldots \ldots \ldots \ldots$

5. Sales of Residual Fuel Oil by End Use, 1995 and $1996 \ldots \ldots \ldots \ldots \ldots \ldots \ldots \ldots \ldots$

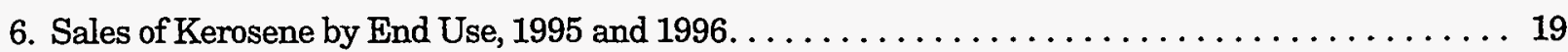

7. Sales for Residential Use: Distillate Fuel Oil and Kerosene, $1996 \ldots \ldots \ldots \ldots \ldots \ldots \ldots \ldots$

8. Sales for Commercial Use: Distillate Fuel Oil, Residual Fuel Oil, and Kerosene, 1996 . . . . . . 22

9. Sales for Industrial Use: Distillate Fuel Oil, Residual Fuel Oil, and Kerosene, 1996 . . . . . . . 23

10. Sales for Farm Use: Distillate Fuel Oil and Kerosene; Sales for Electric Utility and Oil Company Uses: Distillate Fuel Oil and Residual Fuel Oil, 1996 . . . . . . . . . . . . . . . 24

11. Sales for Transportation Use: Distillate Fuel Oil and Residual Fuel Oil, 1996 . . . . . . . . . 25

12. Sales for Military, Off-Highway, and All Other Uses: Distillate Fuel Oil, Residual Fuel

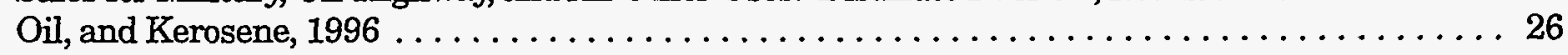

\section{Adjusted Sales of Fuel Oil and Kerosene in 1996}

13. Adjusted Sales of Distillate Fuel Oil by End Use in the United States: $1992-1996$. . . . . . . 28

14. Adjusted Sales of Residual Fuel Oil by End Use in the United States: $1992-1996$. . . . . . . 29

15. Adjusted Sales of Kerosene by End Use in the United States: 1992-1996 . . . . . . . . . . . 29

16. Adjusted Sales of Distillate Fuel Oil by End Use, 1995 and $1996 \ldots \ldots \ldots \ldots \ldots \ldots \ldots$

17. Adjusted Sales of Residual Fuel Oil by End Use, 1995 and $1996 \ldots \ldots \ldots \ldots \ldots \ldots$

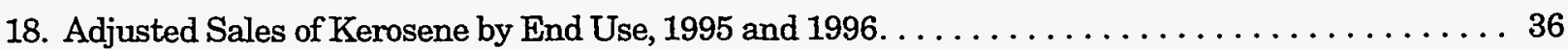

19. Adjusted Sales for Residential Use: Distillate Fuel Oil and Kerosene, 1996 . . . . . . . . . 38

20. Adjusted Sales for Commercial Use: Distillate Fuel Oil, Residual Fuel Oil, and 
21. Adjusted Sales for Industrial Use: Distillate Fuel Oil, Residual Fuel Oil and Kerosene, 1996. . . . 40

22. Adjusted Sales for Farm Use: Distillate Fuel Oil and Kerosene;Adjusted Sales for Electric Utility and Oil Company Uses: Distillate Fuel Oil and Residual Fuel Oil, $1996 \ldots \ldots \ldots \ldots \ldots 1$

23. Adjusted Sales for Transportation Use: Distillate Fuel Oil and Residual Fuel Oil, 1996 . . . . . . 42

24. Adjusted Sales for Military, Off-Highway, and All Other Uses: Distillate Fuel Oil, Residual Fuel Oil, and Kerosene, 1996. 


\section{Introduction}

The Fuel Oil and Kerosene Sales 1996 report provides information, illustrations and State-level statistical data on end-use sales of kerosene; No.1, No. 2, and No. 4 distillate fuel oil; and residual fuel oil. State-level kerosene sales include volumes for residential, commercial, industrial, farm, and all other uses. Statelevel distillate sales include volumes for residential, commercial, industrial, oil company, railroad, vessel bunkering, military, electric utility, farm, on-highway, off highway construction, and other uses. State-level residual fuel sales include volumes for commercial, industrial, oil company, vessel bunkering, military, electric utility, and other uses. The Petroleum Marketing Division, Office of Oil and Gas, Energy Information Administration ensures the accuracy, quality, and confidentiality of the published datain the Fuel Oil and Kerosene Sales 1996.

Except for the kerosene and on-highway diesel information, data presented in Tables 1 through 12 (Sales of Fuel Oil and Kerosene) present results of the EIA-821 survey. Tables 13 through 24 (Adjusted Sales of Fuel Oil and Kerosene) include volumes that are based on the EIA-821 survey but have been adjusted to equal the products supplied volumes published in the Petroleum Supply Annual (PSA).

Tables 1 through 12 contain sales estimates resulting from the EIA-821 survey for all categories exceptkerosene and on-highway diesel. For on-highway diesel the survey was not expected to yield valid statistics because the sampling frame does not include a comprehensive listing of all truck stops. Hence, State-level data obtained from the Federal Highway Administration were used instead.

Kerosene sales data were not expected to be complete because a comprehensive listing of kerosene retailers was not available to serve as a sampling frame. It was thought that a complete frame would be expensive to construct because many kerosene retailers are convenience stores or other small businesses. Because of these concerns, kerosene sales data have been published only after adjusting the sales data so that they add to the U.S. total of kerosene product supplied from the PSA.

In Tables 13 through 24, estimates of distillate fuel oil are adjusted at the Petroleum Administration for Defense (PAD) district level to equal published PSA volume estimates of products supplied. For certain sales categories, data obtained from alternate sources are used instead of the adjusted numbers. See "Technical Note 3" in Appendix for further explanation.

Kerosene and residual fuel oil in Tables 13 through 24 are adjusted at the national level to equal published PSA products supplied estimates. Historically, kerosene estimates have always been adjusted at the PADdistrict level. However, in 1992, certain kerosene estimates at the PAD district level resulted in negative numbers. Hence, the adjustment procedure was changed to display positive sales volumes of kerosene for all PAD districts. Thus the kerosene figures in Tables 13 through 24 are identical to those shown in Tables 1 through 12.

The sales data (Tables 1 through 12) differ from the adjusted sales estimates (Tables 13 through 24) with the exception of kerosene and on-highway diesel for many reasons, including:

- Some products are interchangeable (fungible) and may be supplied as one product and sold as another product. For example, kerosene, low-sulfur kerosene-type jet fuel, and low-sulfur No. 1 fuel oil can be used interchangeably.

- Products supplied into a PAD district may be blended prior to final sale. For example, residual fuel and No. 2 distillate may be blended and sold as No. 4 fuel oil or, in colder climates, kerosene may be blended with distillate fuel oil and sold as heating oil.

- Geographic differences can be attributed to the transportation of product by truck or rail from the district of production. Inter-PAD district movements of products by these modes of transportation are not accounted for in Tables 13 through 24.

- Products may be supplied into a PAD district but the final sale may cross $P A D$ district boundaries. For example, a fuel oil dealer in Ohio (PAD District II) may make retail sales into Pennsylvania (PAD District IB) and/or West Virginia (PAD District IC).

- Drawdowns or buildups in stocks will cause volumes supplied to differ from sales volumes. 


\section{Sales of Fuel Oil and Kerosene in 1996}

Distillate sales rose again in 1996, up 3.7 percent over 1995, propelled upward by an expanding economy. In contrast to 1995, residual fuel oil sales increased in 1996, the first gain since 1988. Residual fuel oil sales increased 7.7 percent over 1995. This shift in the trend of residual fuel sales was the consequence of extremely cold weather during the beginning of 1996 and competitive residual fuel prices toward the end of 1996.

\section{Distillate Fuel Oil}

Distillate sales went from 51.5 billion gallons in 1995 to 53.4 billion gallons in 1996 and experienced an increase in every category except commercial and military use. The critical factors affecting distillate sales in each of these categories were the economy, weather, and crude oil prices. Economic vitality had the most profound influence on distillate sales in the transportation sector.

The transportation sector, consisting of on-highway, vessel bunkering and railroad uses, continued to dominate distillate sales with 61.6 percent of total distillate sales. A strong economy, indicated by a 4.4 percent increase (in current dollars) in the Gross Domestic
Product (GDP), pushed distillate sales in the transportation sector up 5.0 percent in 1996 . At 27.0 billion gallons, on-highway diesel sales increased 5.4 percent to 82.0 percent of distillate in the transportation sector and 50.5 percent of all distillate sales. On-highway sales went up because the demand for delivery of goods and services increased in 1996 . Vessel bunkering and railroad use also both increased in 1996, rising 6.5 and .53 percent respectively. Sales of distillate to railroads increased slightly in 1996 to 3.4 billion gallons, up 18 million gallons. Due to respondent reporting errors in 1995 this increase is actually larger than it appears. Vessel bunkering increased as well to 2.5 billion gallons, an increase of 152 million gallons from 1995.

Despite a colder heating season, distillate sales to residential customers for home heating remained relatively unchanged from 1995. Residential use at 6.9 billion gallons was up only .7 percent in 1996. Approximately 12.9 percent of all distillate sales in 1996 were for residential use and 82.8 percent of those sales were in PAD District I. The principal reason for the consistency in sales was an increase in natural gas consumption stimulated by competitive pricing. While residential heating oil prices increased 10.7 percent in

Table HL1. Volume Distribution of Distillate and Residual Fuel Oils, 1995 and 1996

\begin{tabular}{|c|c|c|c|c|c|c|c|c|}
\hline \multirow[b]{2}{*}{ End Use } & \multicolumn{2}{|c|}{ Distillate 1996} & \multicolumn{2}{|c|}{ Distillate 1995} & \multicolumn{2}{|c|}{ Residual 1996} & \multicolumn{2}{|c|}{ Residual 1995} \\
\hline & $\begin{array}{l}\text { Volume } \\
\text { (million } \\
\text { gallons) }\end{array}$ & $\begin{array}{c}\text { Percent } \\
\text { Share }\end{array}$ & $\begin{array}{c}\text { Volume } \\
\text { (million } \\
\text { gallons) }\end{array}$ & $\begin{array}{c}\text { Percent } \\
\text { Share }\end{array}$ & $\begin{array}{l}\text { Volume } \\
\text { (million } \\
\text { gallons) }\end{array}$ & $\begin{array}{c}\text { Percent } \\
\text { Share }\end{array}$ & $\begin{array}{l}\text { Volume } \\
\text { (million } \\
\text { gallons) }\end{array}$ & $\begin{array}{c}\text { Percent } \\
\text { Share }\end{array}$ \\
\hline Residential $\ldots \ldots \ldots \ldots \ldots \ldots \ldots \ldots$ & 6,910 & 12.9 & 6,860 & 13.3 & -- & - & - & - \\
\hline Commercial.................... & 3,604 & 6.8 & 3,631 & 7.1 & 928 & 7.0 & 923 & 7.5 \\
\hline 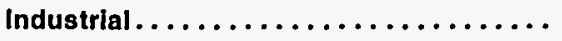 & 2,289 & 4.3 & 2,239 & 4.3 & 2,089 & 15.7 & 1978 & 16.1 \\
\hline 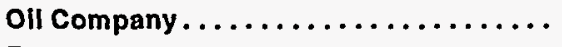 & 731 & 1.4 & 687 & 1.3 & 167 & 1.3 & 211 & 1.7 \\
\hline Farm $\ldots \ldots \ldots \ldots \ldots \ldots \ldots \ldots \ldots \ldots \ldots \ldots$ & 3,591 & 6.7 & 3,476 & 6.8 & -- & -- & -- & - \\
\hline Electric ....................... & 658 & 1.2 & 597 & 1.2 & 4,305 & 32.5 & 3262 & 26.5 \\
\hline 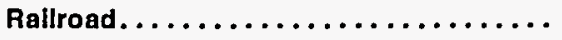 & 3,447 & 6.5 & 3,429 & 6.7 & - & -- & -- & -- \\
\hline 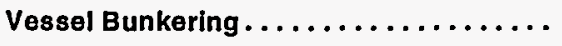 & 2,491 & 4.7 & 2,339 & 4.5 & 5,701 & 43.0 & 5,886 & 47.8 \\
\hline On-Highway $\ldots \ldots \ldots \ldots \ldots \ldots \ldots$ & 26,959 & 50.5 & 25,576 & 49.7 & -- & - & $\cdot-$ & -- \\
\hline 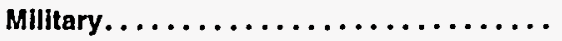 & 454 & 0.8 & 462 & 0.9 & 56 & 0.4 & 51 & 0.4 \\
\hline Off-Highway $\ldots \ldots \ldots \ldots \ldots \ldots \ldots$ & 2,246 & 4.2 & 2,173 & 4.2 & - & -- & -- & -- \\
\hline Other $\ldots \ldots \ldots \ldots \ldots \ldots \ldots \ldots \ldots \ldots \ldots$ & 0 & 0.0 & 0 & 0.0 & 10 & 0.1 & 7 & 0.0 \\
\hline 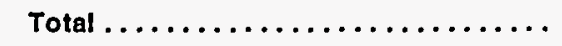 & 53,380 & 100.0 & 51,469 & 100.0 & 13,256 & 100.0 & 12,318 & 100.0 \\
\hline
\end{tabular}

Sources: Energy Information Administration, Form ElA-821, "Annual Fuel Oil and Kerosene Sales Report," 1995 and 1996. 
1996, natural gas prices only increased 0.8 percent. $^{1}$ Heating oil prices remained high because the cold fall season in 1995 hindered the typical rise in stock levels. The high price of crude oil and persistent expectations that the price would drop helped maintain the low levels of distillate stock throughout the year. ${ }^{2}$ Additionally, more efficient inventory management and a strategy of just in time inventorying also contributed to low stock levels. Diminishing residential distillate sales, however, were offset by a 6.9 percent increase in residential and commercial natural gas consumption. ${ }^{3}$ Residential natural gas should continue to curtail the heating oil share of the market as more existing homes convert to natural gas and the quantity of new gas home completions rises.

Commercial use distillate decreased 7 percent in 1996 , which equates to a decrease of 27 million gallons. The largest decrease was in low sulfur diesel which decreased 111 million gallons or 9.3 percent in 1996 . Decreased use of on-highway diesel by governments was the primary reason for this drop.

Distillate sales for industrial use increased 2.2 percent in 1996. In order to satisfy escalating demand, industrial use sales increased by 50 million gallons. A growing economy evidenced by an increase in manufacturing production and capacity utilization of 2.74 percent, contributed to the increase. ${ }^{4}$

Farm use of distillate at 3.6 billion gallons was up 3.3 percent in 1996. The principle reason for this increase was the total area planted (in principal crops) rising 8.3 percent. $^{5}$ Some gain in fuel sales was due to an increase in the fuel requirements of those particular commodities planted and harvested. The area of corn and wheat planted increased 13.0 and 8.6 percent, respectively, and had the greatest impact on the increase in total area planted. ${ }^{6}$ In fact, the area planted for spring wheat was the most since 1936. The area planted for corn increased by 9 million acres and corn is a fuel intensive commodity. The total area of corn harvested was 74 million acres and was the largest corn crop since 1985. The tremendous growth in the area planted for corn and wheat was offset moderately, however, by a 15.2 percent decrease in area planted for cotton.

Off-Highway construction and other uses were up 3.4 percent in 1996 to 2.2 billion gallons, primarily because new private construction increased 4.3 percent. ${ }^{8}$ Any government use of fuel for construction is categorized under military or commercial end-use.

Oil company use was up 6.4 percent in 1996 due to an increase in drilling activities in the United States. The number of rotary rigs in operation increased by 7.8 percent in 1996, and the total footage drilled also climbed 22.5 percent. ${ }^{9}$ This growth resulted from the 26.3 percent increase in the price of crude oil in 1996 which motivated oil companies to increase their exploration and development expenditures.

Electric Utility sales increased 10.1 percent in 1996 to 658 million gallons, an increase of 60 million gallons. This increase was the result of a 3.3 percent increase in heating degree days and an increase in weather volatility over $1995 .^{10}$ The severely cold weather during the initial few months of 1996 increased energy consumption and the demand for peak use diesel fired generation units. Historically, there is little demand for distillate fuel oil in warm or normal weather conditions.

Military distillate use dropped 1.6 percent in 1996 to 454 million gallons due to a decline in military activity and the expanded use of JP-8 fuel. JP-8 is a kerosene based jet fuel currently being used as a substitute for diesel in many military vehicles. By using this one standard fuel in all of its equipment, the military will vastly improve logistical efficiency, reliability, and mobility. Military distillate use should continue to dimin-

\footnotetext{
1Monthly Energy Review (MER), June 1997, Table 1.7, p.13.

2Distillate Fuel Oil Assessment for Winter 1996-1997, Graig H. Cranston, EIA, p.7.

${ }^{3}$ Monthly Energy Review (MER), June 1997, Table 2.3, p.27.

${ }^{4}$ Economic Indicators, May 1997, Washington D.C. US Government Printing Office, p.17.

бbid.

Tbid.

8Economic Indicators, May 1997, Washington D.C. US Government Printing Office, p.19.

9Monthly Energy Review (MER), August 1996, Table 5.1, p.82.

${ }^{10}$ Short-Term Energy Outlook, Third Quarter 1997, p.22.
}

sAcreage planted figures come from the Annual Crop Summary, Agricultural Statistics Board, NASS, USDA, June 1996. 
ish as the military pursues this transition toward a single fuel force employing the more stable and reliable JP-8 fuel.

\section{Residual Fuel Oil}

In 1996, residual fuel oil sales in the United States increased for the first year since 1988. Residual fuel oil sales went from 12.3 billion gallons in 1995 to 13.3 billion gallons in 1996. This 7.7 percent increase was driven by a 31.9 percent increase in electric utility sales.

Vessel bunkering maintained its dominance by securing 43.0 percent of the residual fuel market, in spite of a decline in sales of 3.0 percent. PAD District V had the most substantial effect on this decrease with a loss of 14.4 percent in 1996. This decrease was due to a notable increase in exports to Asia where poor availability of residual fuel oil stimulated demand. While PAD District V witnessed a decrease in sales, more competitive prices spurred sales upwards slightly in PAD Districts I, II, and III. Throughout the majority of 1996, the U.S. Gulf maintained a favorable price advantage over Rotterdam compared to 1995 and this promoted the increase in sales. Overall, the decrease in sales of residual fuel oil was influenced by the unusually high demand for residual fuel in the utility market. Despite the decline in vessel bunkering sales, total residual fuel oil sales were propelled upwards by this increase in electric utility sales.

Electric utility sales accounted for 32.4 percent of all residual fuel oil sales, up from the 26.5 percent market share in 1995 . Sales escalated by 1.0 billion gallons last year, rising from only 3.3 billion gallons in 1995 to 4.3 billion gallons in 1996 . Three principle factors account for this dramatic shift upwards in sales. First, the harsh cold weather in the Northeast and Mississippi during the first quarter of 1996 elevated electric utility demand for residual fuel oil. When unusually cold weather struck Mississippi and froze gas pipes, some utilities had to increase their reliance on residual fuel oil. Then, Northeast Nuclear Energy was forced to shutdown its three Millstone plants in March of 1996 and their Connecticut Yankee plant in July because of safety violations. This substantial drop in nuclear power plants stirred demand for residual fuel oil. In Connecticut, residual fuel oil sales climbed 328 million gallons over 1995. Lastly, through November and December of 1996 natural gas prices rose 68 percent and competitive pricing of residual fuel oil encouraged electric utilities to use more residual fuel oil. ${ }^{11}$
Industrial remained the third largest end use category for residual fuel oil sales of 2.1 billion gallons in 1996 . Industrial use increased by 112 million gallons, a gradual increase of 5.7 percent. General increased demand for industrial goods had a direct effect on the increase. Like electric utility sales, this increase was also due in part to the low price of residual fuel oil compared to natural gas toward the end of 1996. Futhermore, as the year progressed and natural gas supplies decreased, companies with interruptible contracts were forced to switch to residual fuel oil.

Commercial use of residual fuel oil increased .57 percent in 1996 to 928 million gallons. Increased demand for heating in 1996 was the key reason for the increase. Although the number of apartment buildings and commercial facilities heated with residual fuel oil is declining, the cold weather increased overall demand.

Oil company use of residual fuel continued its decline in 1996 by dropping 20.8 percent from 211 million to 167 million gallons. As refineries adopt new technologies which reduce the amount of residual fuel produced, oil companies will continue to use less residual fuel oil for operations. The unusually high demand for residual fuel oil by utilities also contributed to the decrease.

Military use of residual fuel oil increased 10.5 percent in 1996 to 56 million gallons, an increase of 5 million gallons. The greatest increases occurred in Texas and Hawaii as the military replenished residual fuel stock levels in those states.

\section{Kerosene}

Total U.S. kerosene sales increased 14.6 percent in 1996. Increases were observed in the residential, industrial, and farm use sectors. Residences continued to use the majority of kerosene, with 69.3 percent of all kerosene sales. At 658 million gallons, residential use increased 19.5 percent in 1996, primarily due to weather conditions throughout PAD District I. PAD District I dominated the residential sector with 80.7 percent of all residential kerosene sales and experienced a 20.4 percent increase in 1996. The greatest impact on the increase in kerosene sales, however, was experienced by the farm use sector. Kerosene sales for farm use increased 30.6 percent in 1996.

"Natural Gas Monthly, July 1997, Table 4., p.12. 


$$
\text { E }
$$


Figure HL1. U.S. Sales of Distillate and Residual Fuel Oils by End Use, 1994-1996
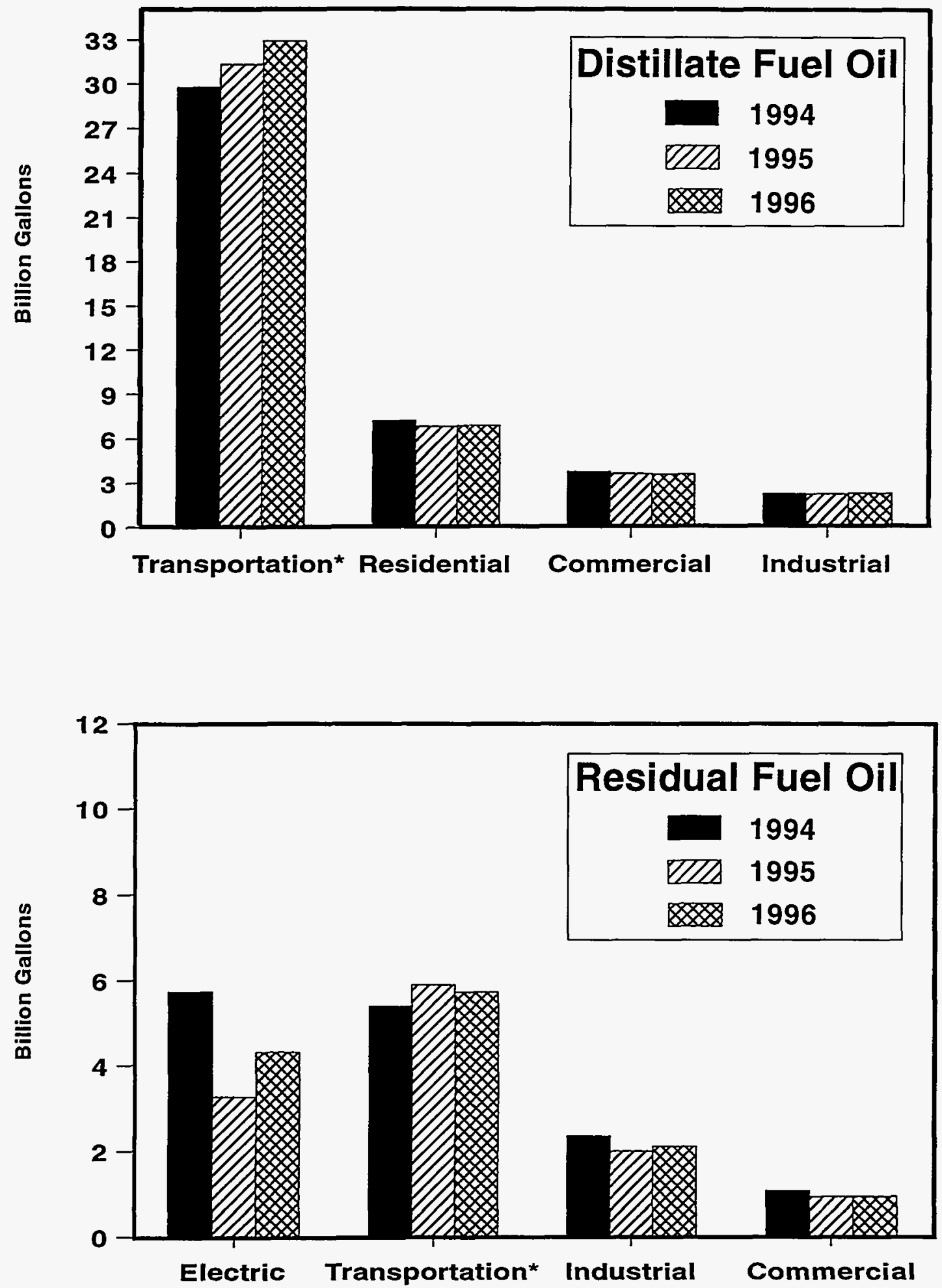

-For distillate fuel oil, transportation use comprises railroad, vessel bunkering, and on-highway diesel

end-use categories. For residual fuel oil, transportation use comprises the vessel bunkering end-use category.
Sources: Energy Information Administration, Form EIA-821, "Fuel Oil and Kerosene Sales Report," 1995 and 1996. 
Figure HL2. Volume Distribution of Distillate and Residual Fuel Oils by PAD District, 1994-1996
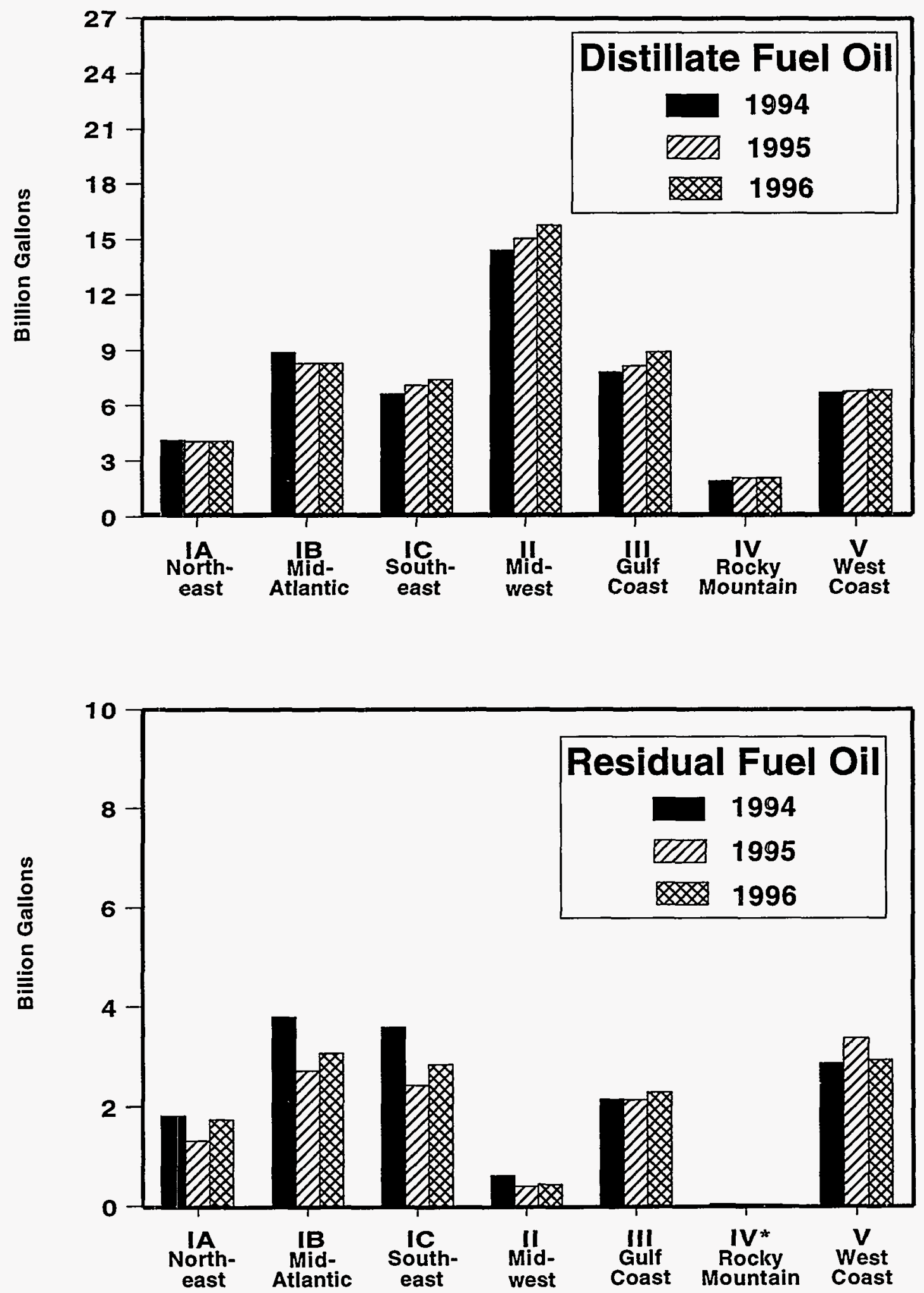

* Residual fuel oil sales in PAD District IV are too small to appear in this graph.

Sources: Energy Information Administration, Form EIA-821, "Fuel Oil and Kerosene Sales Report," 1995 and 1996. 
Figure HL3. Distillate Sales for Transportation End-Use by PAD District and State, 1996
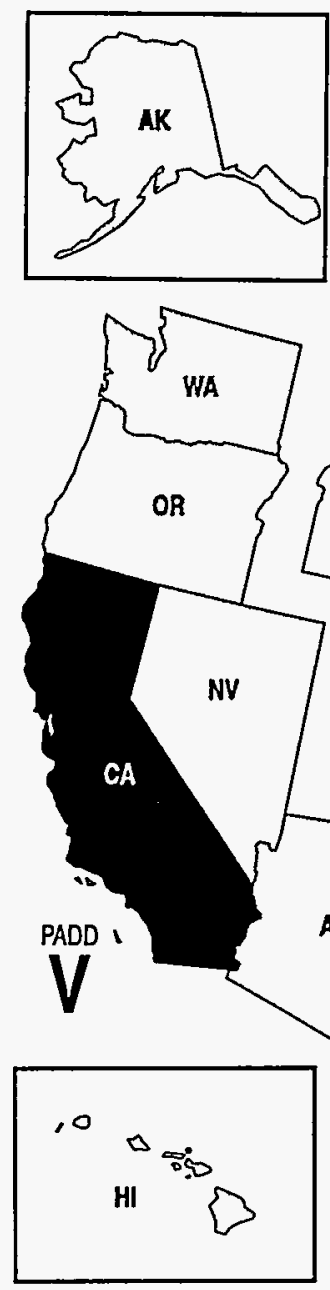

IV

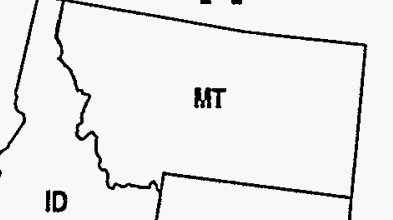

wY

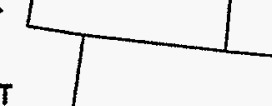

UT $\quad$ Co
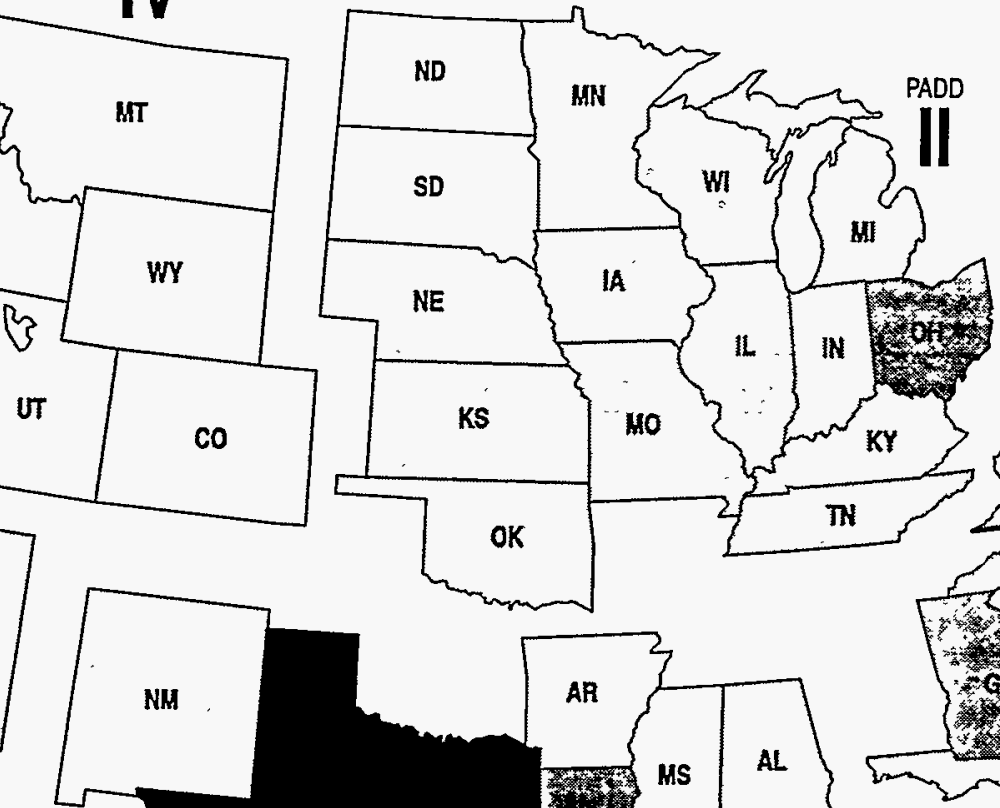

V
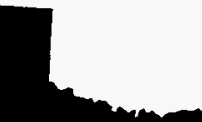

$\mathrm{TX}$

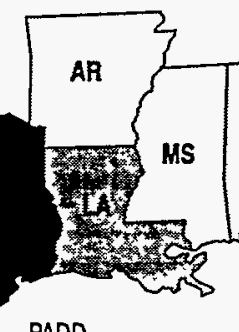

PADD

III

Shading Represents State Sales

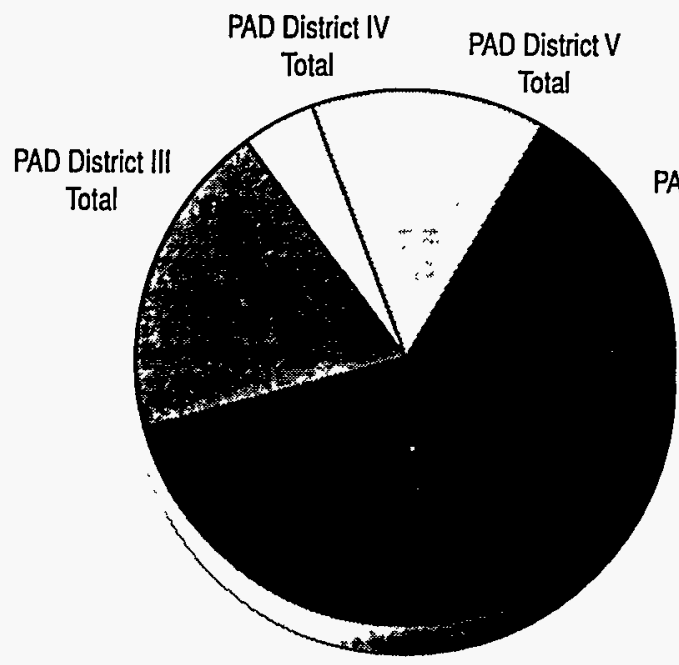

PAD District II

Total
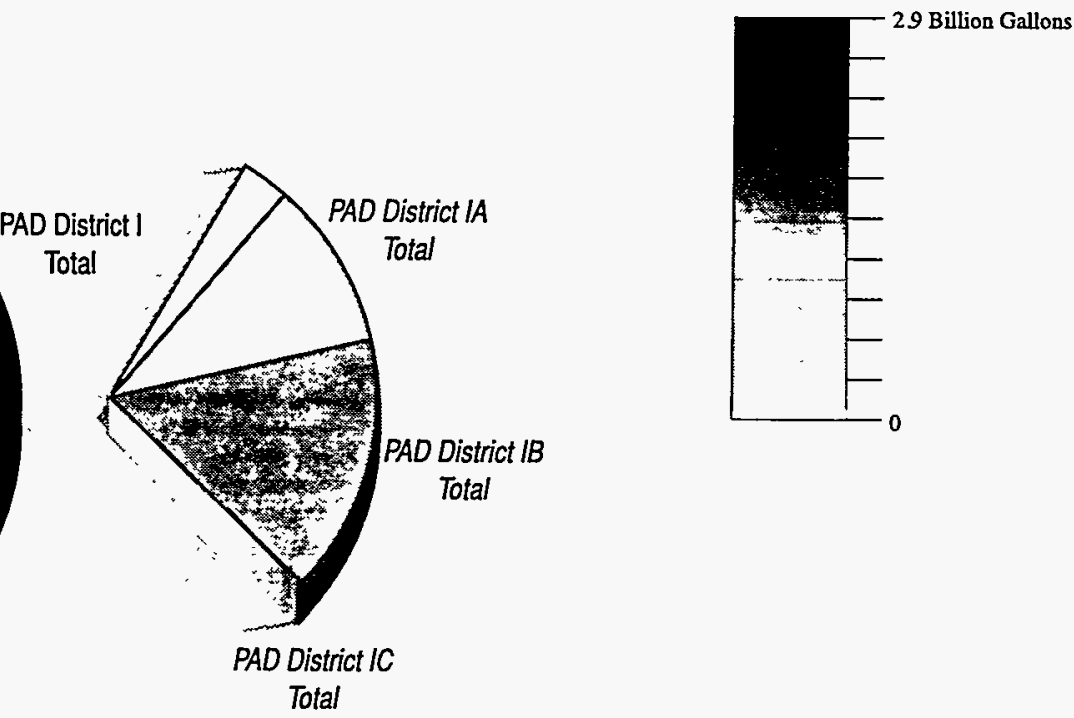

Sources: Energy Information Administration, Form EIA-821, "Fuel Oil and Kerosene Sales Report," 1996. 
Figure HL4. Residual Fuel Oil Sales for Electric Utility End-Use by PAD District and State, 1996

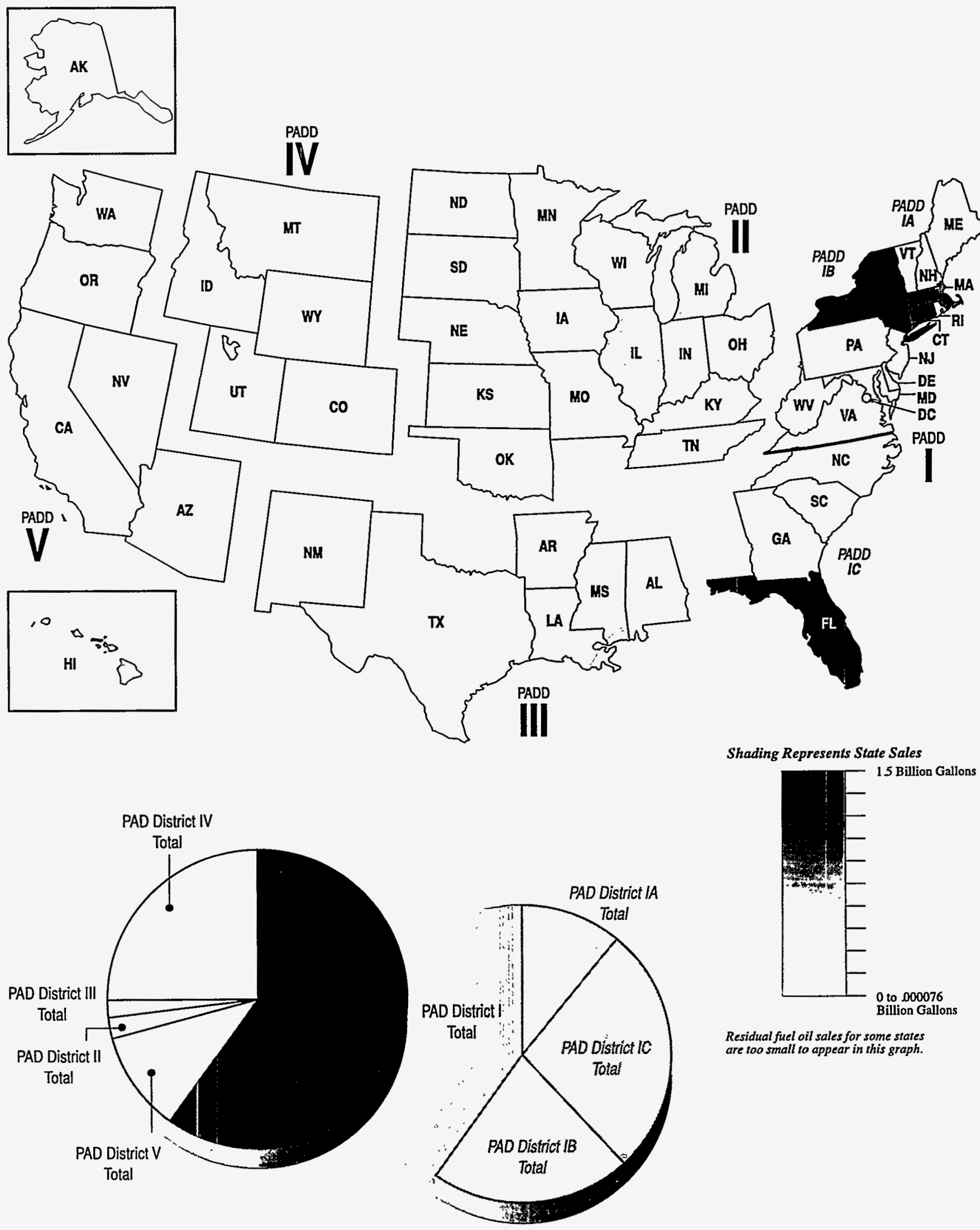

Sources: Energy Information Administration, Form ElA-821, "Fuel Oil and Kerosene Sales Report," 1996. 
Table 1. Sales of Distillate Fuel Oil by End Use in the United States: $1992-1996$

(Thousand Gallons)

\begin{tabular}{|c|c|c|c|c|c|}
\hline \multirow{2}{*}{ End Use } & \multicolumn{5}{|c|}{ Distillate Fuel Oll } \\
\hline & 1992 & 1993 & 1994 & 1995 & 1996 \\
\hline 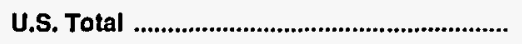 & $47,261,552$ & $48,290,076$ & $50,423,596$ & $51,469,264$ & $53,379,033$ \\
\hline 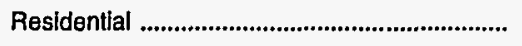 & $7,291,444$ & $7,287,196$ & $7,232,733$ & $6,859,786$ & $6,910,018$ \\
\hline 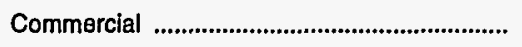 & $3,771,021$ & $3,689,509$ & $3,774,929$ & $3,630,856$ & $3,603,620$ \\
\hline Industrial & $2,312,146$ & $2,130,358$ & $2,269,007$ & $2,238,873$ & $2,288,689$ \\
\hline Oil Company & 664,408 & 709,404 & 675,228 & 686,750 & 730,676 \\
\hline 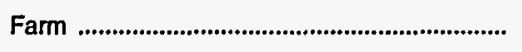 & $3,499,518$ & $3,410,827$ & $3,270,227$ & $3,476,472$ & $3,591,383$ \\
\hline 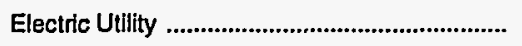 & 540,683 & 550,752 & 663,814 & 597,461 & 657,881 \\
\hline Railroad & $3,172,724$ & $3,000,104$ & $3,141,324$ & $3,428,829$ & $3,446,939$ \\
\hline 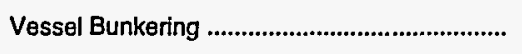 & $2,218,592$ & $2,154,757$ & $2,189,346$ & $2,339,010$ & $2,490,793$ \\
\hline 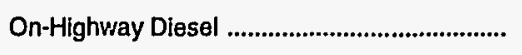 & $21,374,872$ & $22,758,842$ & $24,442,609$ & $25,576,433$ & $26,958,668$ \\
\hline 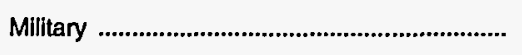 & 652,507 & 491,320 & 610,474 & 461,740 & 454,443 \\
\hline 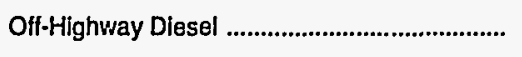 & $1,757,788$ & $2,104,299$ & $2,153,153$ & $2,173,054$ & $2,245,922$ \\
\hline All Other & 5,848 & 2,709 & 752 & - & - \\
\hline
\end{tabular}

Dashes $(-)=$ No data reported.

Note: Totals may not equal sum of components due to independent rounding

Sources: - Energy Information Administration Form EIA-821, "Annual Fuel Oil and Kerosene Sales Report," for $1992-1996$.

- On-Highway Diesel data are derived from Federal Highway Administation statistics of highway special fuels use.

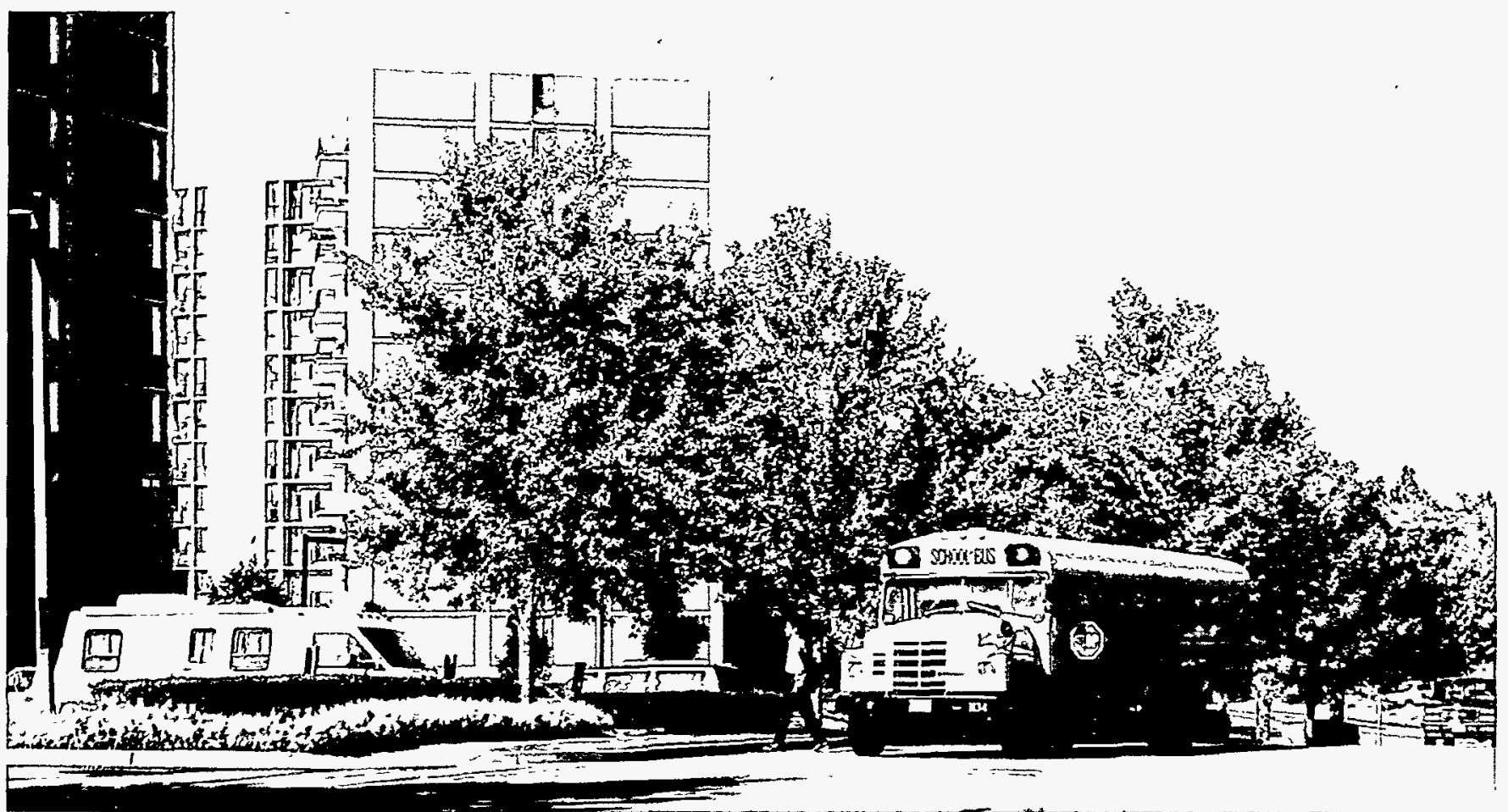

Sales to school buses and apartment buildings are considered commercial use. 
Table 2. Sales of Residual Fuel Oil by End Use in the United States: 1992-1996 (Thousand Gallons)

\begin{tabular}{|c|c|c|c|c|c|}
\hline \multirow{2}{*}{ End Use } & \multicolumn{5}{|c|}{ Residual Fuel Oil } \\
\hline & 1992 & 1993 & 1994 & 1995 & 1996 \\
\hline U.S. Total & $16,199,042$ & $15,064,305$ & $14,825,378$ & $12,318,128$ & $13,256,998$ \\
\hline 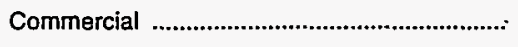 & $1,176,049$ & $1,025,895$ & $1,061,450$ & 922,572 & 927,834 \\
\hline 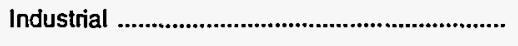 & $2,126,857$ & $2,378,191$ & $2,337,188$ & $1,977,567$ & $2,089,436$ \\
\hline 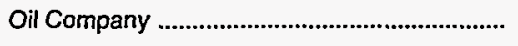 & 271,070 & 266,518 & 242,754 & 211,450 & 167,425 \\
\hline Electric Utility & $5,961,441$ & $6,035,413$ & $5,729,485$ & $3,262,492$ & $4,304,492$ \\
\hline 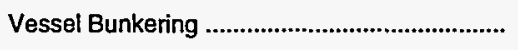 & $6,563,220$ & $5,281,869$ & $5,386,407$ & $5,886,409$ & $5,701,233$ \\
\hline Military & 91.791 & 72,540 & 61,946 & 50,902 & 56,225 \\
\hline 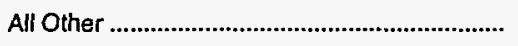 & 8,613 & 3,878 & 6,111 & 6,735 & 10,354 \\
\hline
\end{tabular}

Note: Totals may not equal sum of components due to independent rounding.

Sources: - Energy Information Administration Form ElA-821, "Annual Fuel Oil and Kerosene Sales Report," for $1992-1996$.

Table 3. Sales of Kerosene by End Use in the United States: 1992-1996 (Thousand Gallons)

\begin{tabular}{|c|c|c|c|c|c|}
\hline \multirow{2}{*}{ End Use } & \multicolumn{5}{|c|}{ Kerosene } \\
\hline & 1992 & 1998 & 1994 & 1995 & 1996 \\
\hline U.S. Total & 636,426 & 760,788 & 750,330 & 828,450 & 948,990 \\
\hline 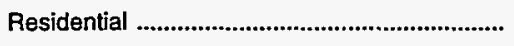 & 481,511 & 560,103 & 480,916 & 550,336 & 657,636 \\
\hline 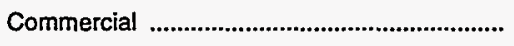 & 82,398 & 103,996 & 144,142 & 163,716 & 155,792 \\
\hline 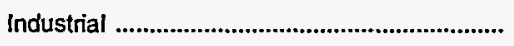 & 46,099 & 58,190 & 86,629 & 86,902 & 102,322 \\
\hline 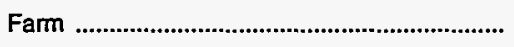 & 20,036 & 27,695 & 27,686 & 19,919 & 26,017 \\
\hline All Other & 6,382 & 10,805 & 10,957 & 7,576 & 7,223 \\
\hline
\end{tabular}

Note: Totals may not equal sum of components due to independent rounding.

Note: Kerosene data in the Sales tables (1-12) are adjusted at the national level. See Technical Note 3 for further explanation.

Sources: - Kerosene data are based on data from the Energy Information Administration's "Petroleum Supply Annual," Volume 1, $1992-1996$ (DOE/EIA-0340(92)/1 - (96)/1). 
Table 4. Sales of Distillate Fuel Oil by End Use, 1995 and 1996 (Thousand Gallons)

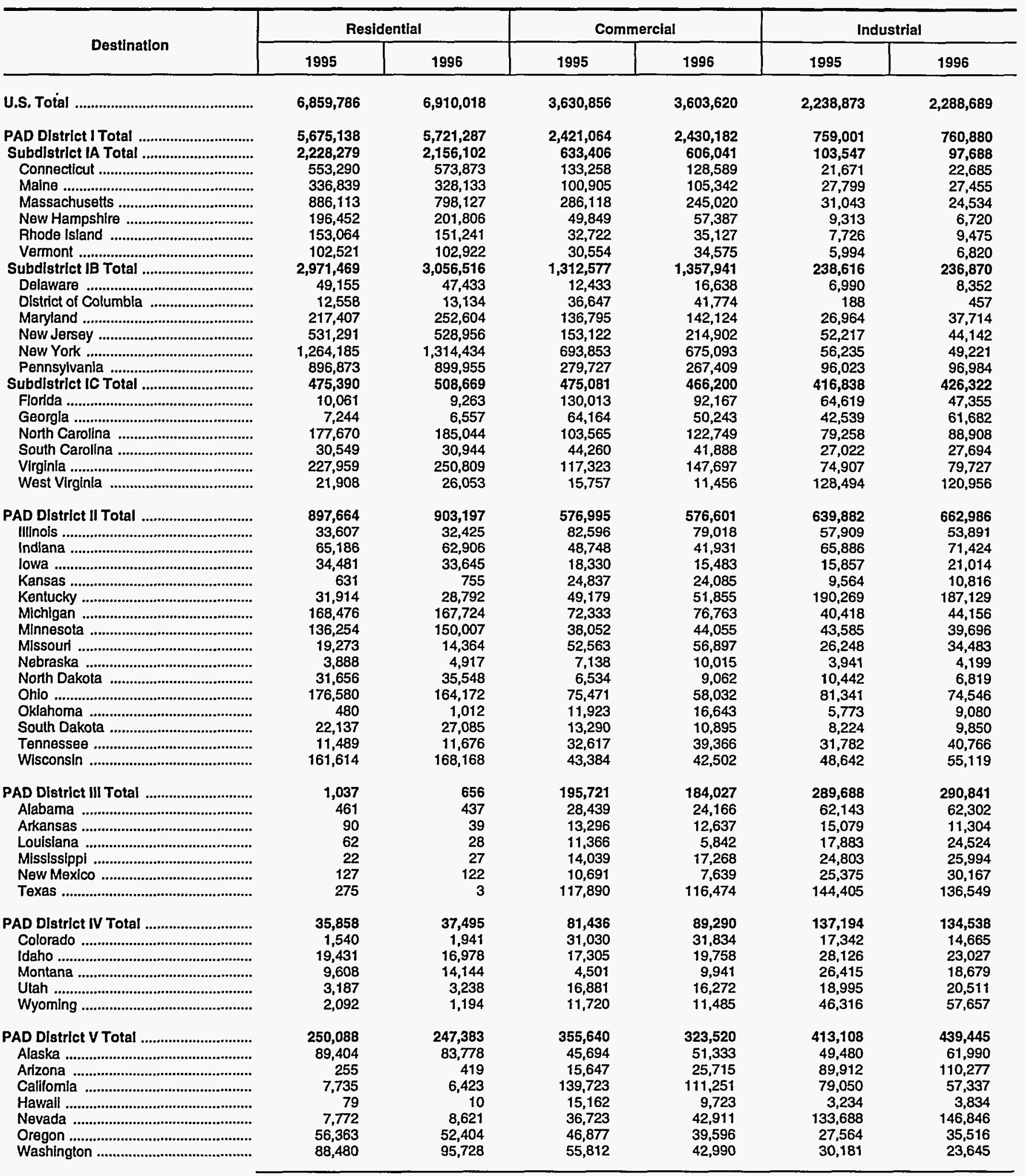

See footnotes at end of table. 
Table 4. Sales of Distillate Fuel Oil by End Use, 1995 and 1996 (Continued) (Thousand Gallons)

\begin{tabular}{|c|c|c|c|c|c|c|}
\hline \multirow{2}{*}{ Destination } & \multicolumn{2}{|c|}{ Oll Company } & \multicolumn{2}{|c|}{ Farm } & \multicolumn{2}{|c|}{ Electric UtHity } \\
\hline & 1995 & 1996 & 1995 & 1996 & 1995 & 1996 \\
\hline U.S. Total & 686,750 & 730,676 & $3,476,472$ & $3,591,383$ & 597,461 & 657,881 \\
\hline 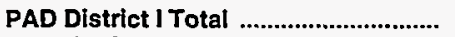 & 10,363 & 9,932 & 397,145 & 409,674 & 257,997 & 272,879 \\
\hline 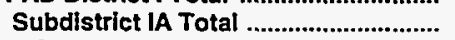 & 1,423 & 558 & 17,465 & 16,587 & 21,866 & 20,018 \\
\hline 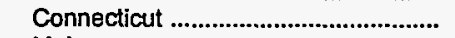 & 389 & 235 & 5,469 & 2,659 & 2,858 & 1,663 \\
\hline Maine & 87 & - & 3,810 & 3,797 & 1,051 & 845 \\
\hline 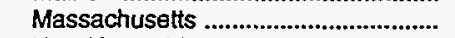 & 480 & 229 & 2,241 & 4,148 & 11,366 & 12,099 \\
\hline New Hampshire ..................................... & - & - & 985 & 926 & 1,204 & 1,609 \\
\hline 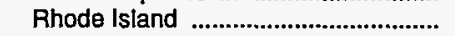 & 444 & 91 & 54 & 100 & 5,054 & 3,741 \\
\hline 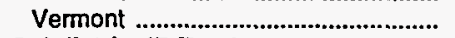 & 22 & 2 & 4,906 & 4,957 & 333 & 62 \\
\hline 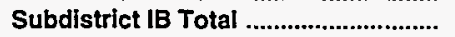 & 3,623 & 4,445 & 104,672 & 108,154 & 112,865 & 106,046 \\
\hline 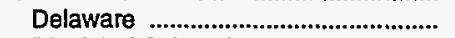 & 305 & 769 & 3,209 & 5,647 & 3,118 & 8,016 \\
\hline District of Columbia & - & - & - & - & 23,567 & 16,587 \\
\hline 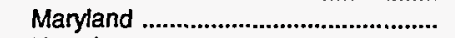 & 616 & 134 & 26,787 & 26,691 & 27,029 & 23,278 \\
\hline 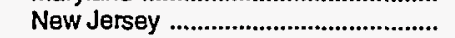 & 931 & 1,522 & 7,108 & 7,300 & 3,895 & 4,974 \\
\hline 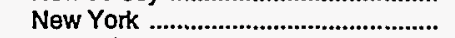 & 1,255 & 1,017 & 34,203 & 33,839 & 9,203 & 14,299 \\
\hline 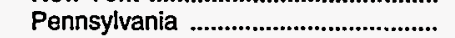 & 515 & 1,003 & 33,367 & 34,676 & 46,054 & 38,891 \\
\hline 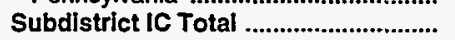 & 5,317 & 4,930 & 275,007 & 284,933 & 123,266 & 146,815 \\
\hline 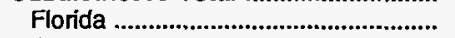 & 1,155 & 560 & 68,083 & 76,350 & 53,446 & 67,739 \\
\hline Georgia & 1,366 & 1,280 & 101,034 & 102,239 & 16,270 & 26,214 \\
\hline North Carolina & 140 & 115 & 44,385 & 34,423 & 22,352 & 22,454 \\
\hline 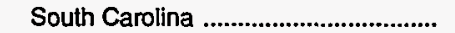 & 1 & 416 & 16,690 & 24,414 & 12,743 & 8,364 \\
\hline 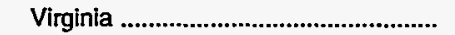 & 1,983 & 1,226 & 40,687 & 43,275 & 7,231 & 9,831 \\
\hline West Virginia & 672 & 1,333 & 4,129 & 4,232 & 11,225 & 12,213 \\
\hline PAD Distriet || Total .............................. & 47,290 & 49,821 & $1,725,632$ & $1,696,468$ & 135,966 & 158,398 \\
\hline 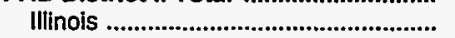 & 2,444 & 1,364 & 221,678 & 207,442 & 25,667 & 29,294 \\
\hline Indiana ................................................. & 2,211 & 2,843 & 110,926 & 94,802 & 16,839 & 17,663 \\
\hline lowa & 6 & 10 & 181,808 & 208,617 & 2,183 & 2,357 \\
\hline 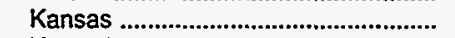 & 5,012 & 4,967 & 166,519 & 165,362 & 2,647 & 6,028 \\
\hline 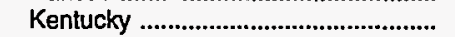 & 585 & 306 & 39,165 & 32,554 & 9,035 & 8,708 \\
\hline 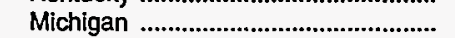 & 2,203 & 2,017 & 60,078 & 64,427 & 23,238 & 24,541 \\
\hline 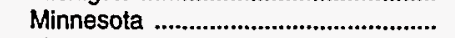 & 68 & - & 173,945 & 182,324 & 4,906 & 9,470 \\
\hline 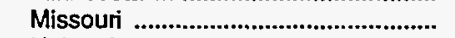 & 55 & 40 & 60,615 & 59,398 & 6,293 & 5,102 \\
\hline 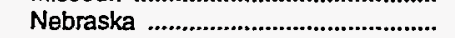 & 774 & 628 & 186,677 & 177,287 & 1,594 & 1,451 \\
\hline North Dakota & 6,208 & 8,449 & 108,785 & 105,271 & 3,945 & 5,293 \\
\hline Ohio & 2,172 & 1,159 & 101,864 & 94,195 & 21,569 & 21,594 \\
\hline 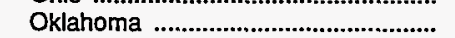 & 25,412 & 27,858 & 73,958 & 77,087 & 885 & 389 \\
\hline South Dakota & 29 & 79 & 80,066 & 80,785 & 278 & 1,193 \\
\hline 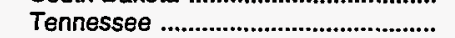 & 108 & 100 & 65,524 & 58,642 & 9,825 & 17,297 \\
\hline Wisconsin & 2 & 2 & 94,022 & 88,273 & 7,062 & 8,017 \\
\hline 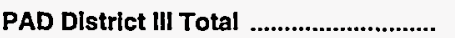 & 547,164 & 570,782 & 742,083 & 842,036 & 45,745 & 62,717 \\
\hline Alabama & 9,429 & 9,902 & 50,169 & 37,780 & 8,180 & 12,298 \\
\hline 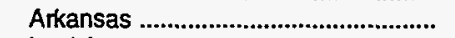 & 1,349 & 1,672 & 123,348 & 107,664 & 9,954 & 5,412 \\
\hline 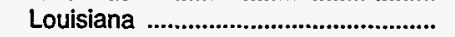 & 329,392 & 339,031 & 70,393 & 62,143 & 8,578 & 18,006 \\
\hline 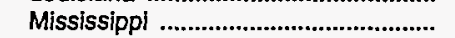 & 2,458 & 1,329 & 105,755 & 95,195 & 1,539 & 4,265 \\
\hline 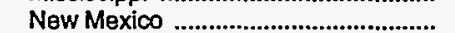 & 23,829 & 19,861 & 17,064 & 17,685 & 1,192 & 1,500 \\
\hline Texas & 180,706 & 198,986 & 375,353 & 521,569 & 16,302 & 21,236 \\
\hline 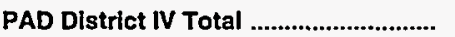 & 24,161 & 25,986 & 188,770 & 204,954 & 5,122 & 6,066 \\
\hline 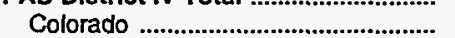 & 9,075 & 7,742 & 55,982 & 68,033 & 661 & 858 \\
\hline Idaho & - & 2 & 48,785 & 46,361 & 350 & 333 \\
\hline 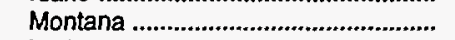 & 1,201 & 1,418 & 58,017 & 64,996 & 38 & 63 \\
\hline 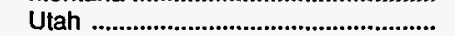 & 3,513 & 4,268 & 14,228 & 14,700 & 1,691 & 2,149 \\
\hline 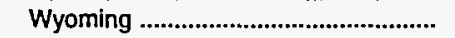 & 10,373 & 12,557 & 11,759 & 10,864 & 2,383 & 2,663 \\
\hline 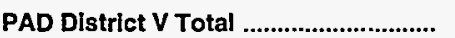 & 57,772 & 74,155 & 422,842 & 438,252 & 152,631 & 157,822 \\
\hline 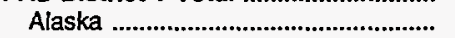 & 46,571 & 66,990 & 158 & 2 & 35,713 & 37,660 \\
\hline Arizona & 267 & 100 & 28,950 & 27,380 & 2,370 & 2,497 \\
\hline 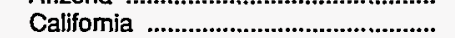 & 9,717 & 5,093 & 256,297 & 289,264 & 4,289 & 4,107 \\
\hline Hawail & 997 & 664 & 6,440 & 8,462 & 108,713 & 108,863 \\
\hline 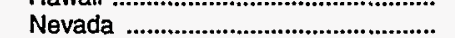 & 74 & 505 & 4,397 & 6,472 & 501 & 1,792 \\
\hline 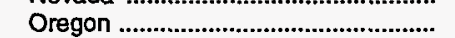 & 5 & 51 & 60,973 & 39,673 & 825 & 825 \\
\hline 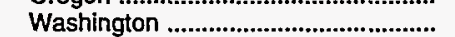 & 141 & 752 & 65,627 & 66,999 & 220 & 2,078 \\
\hline
\end{tabular}

See footnotes at end of table. 
Table 4. Sales of Distillate Fuel Oil by End Use, 1995 and 1996 (Continued) (Thousand Gallons)

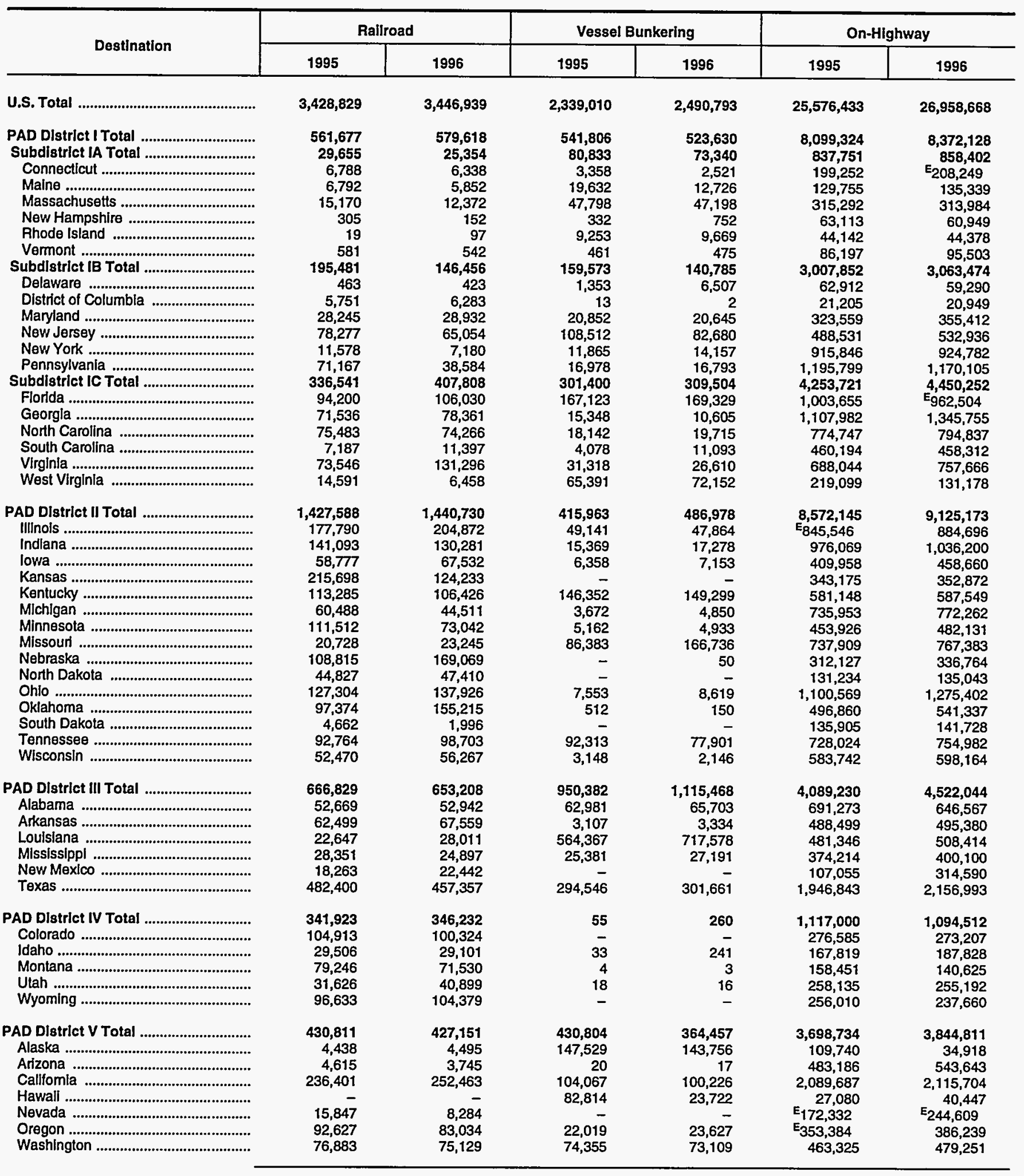

See footnotes at end of table. 
Table 4. Sales of Distillate Fuel Oil by End Use, 1995 and 1996 (Continued) (Thousand Gallons)

\begin{tabular}{|c|c|c|c|c|c|c|c|c|}
\hline \multirow{2}{*}{ Destination } & \multicolumn{2}{|c|}{ Military } & \multicolumn{2}{|c|}{ Off-Highway } & \multicolumn{2}{|c|}{ All Other } & \multicolumn{2}{|c|}{ Total } \\
\hline & 1995 & 1996 & 1995 & 1996 & 1995 & 1996 & 1995 & 1996 \\
\hline U.S. Total & 461,740 & 454,443 & $2,173,054$ & $2,245,922$ & - & - & $51,469,264$ & $53,379,033$ \\
\hline 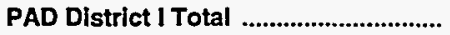 & 98,681 & 81,726 & 609,920 & 624,949 & - & - & $19,432,116$ & $19,786,887$ \\
\hline 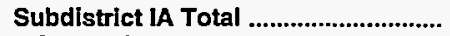 & 19,724 & 11,281 & 70,646 & 75,466 & - & - & $4,044,594$ & $3,940,837$ \\
\hline 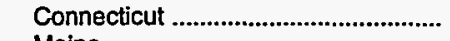 & 660 & 3,945 & 10,101 & 9,660 & - & - & 937,093 & 960,417 \\
\hline 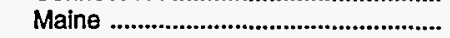 & 2,725 & 3,599 & 21,355 & 26,799 & - & - & 650,750 & 649,888 \\
\hline 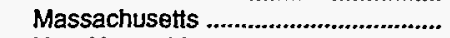 & 9,524 & 1,459 & 22,667 & 24,077 & - & - & $1,627,812$ & $1,483,248$ \\
\hline 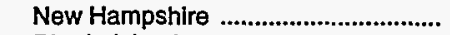 & 1,291 & 54 & 8,825 & 9,450 & - & - & 331,669 & 339,803 \\
\hline 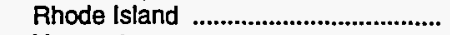 & 5,253 & 1,933 & 4,148 & 3,092 & - & - & 261,880 & 258,943 \\
\hline 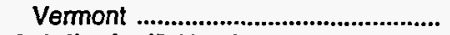 & 270 & 291 & 3,550 & 2,389 & - & - & 235,390 & 248,538 \\
\hline 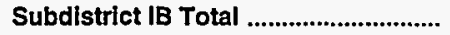 & 25,405 & 37,931 & 163,188 & 173,025 & - & - & $8,295,323$ & $8,431,642$ \\
\hline 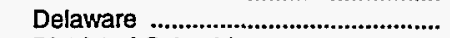 & 1,200 & 1,355 & 4,471 & 7,117 & - & - & 145,608 & 161,547 \\
\hline 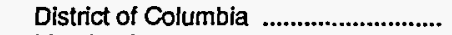 & 1,050 & 2,048 & 503 & 326 & - & - & 101,482 & 101,561 \\
\hline 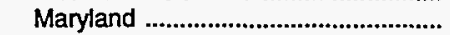 & 13,521 & 18,370 & 22,366 & 24,887 & - & - & 844,141 & 930,794 \\
\hline 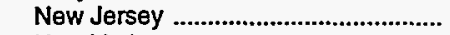 & 814 & 1,972 & 27,829 & 30,784 & - & - & $1,452,526$ & $1,515,222$ \\
\hline 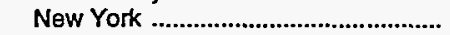 & 2,112 & 2,401 & 43,930 & 48,629 & - & - & $3,044,266$ & $3,085,052$ \\
\hline 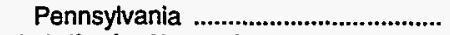 & 6,709 & 11,785 & 64,088 & 61,282 & - & - & $2,707,300$ & $2,637,466$ \\
\hline 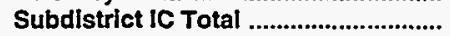 & 53,551 & 32,514 & 376,086 & $\mathbf{3 7 6 , 4 5 9}$ & - & - & $7,092,200$ & $\mathbf{7 , 4 1 4 , 4 0 7}$ \\
\hline 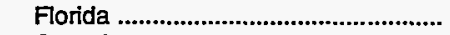 & 12,034 & 7,412 & 121,943 & 121,285 & - & - & $1,726,331$ & $1,659,994$ \\
\hline 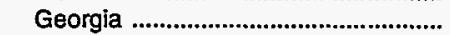 & 10,834 & 3,037 & 75,427 & 73,161 & - & - & $1,513,743$ & $1,759,135$ \\
\hline 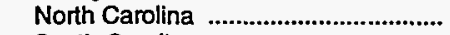 & 8,512 & 3,946 & 81,136 & 66,580 & - & - & $1,385,389$ & $1,413,037$ \\
\hline South Carolina .............................................. & 1,250 & 1,981 & 40,370 & 39,822 & - & - & 644,343 & 656,326 \\
\hline 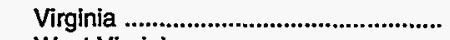 & 20,517 & 15,562 & 44,114 & 65,571 & - & - & $1,327,628$ & $1,529,269$ \\
\hline 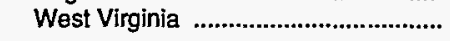 & 405 & 576 & 13,096 & 10,040 & - & - & 494,766 & 396,646 \\
\hline 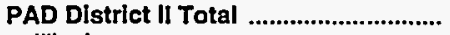 & 16,026 & 22,269 & 599,047 & 649,150 & - & - & $15,054,198$ & $15,771,771$ \\
\hline 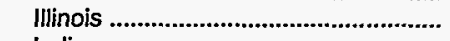 & 424 & 1,428 & 64,471 & 71,593 & - & - & $1,561,274$ & $1,613,887$ \\
\hline Indiana & 652 & 1,893 & 31,444 & 33,958 & - & - & $1,474,424$ & $1,511,180$ \\
\hline lowa & 199 & 228 & 51,230 & 41,918 & - & - & 779,188 & 856,619 \\
\hline 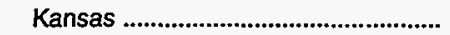 & 1,062 & 943 & 31,689 & 28,577 & - & - & 800,834 & 718,638 \\
\hline 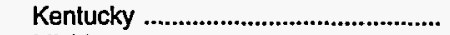 & 2,161 & 1,408 & 40,256 & 45,018 & - & - & $1,203,350$ & $1,199,043$ \\
\hline 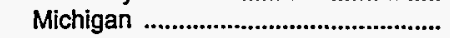 & 352 & 1,627 & 49,973 & 58,458 & - & - & $1,217,182$ & $1,261,334$ \\
\hline 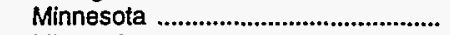 & 259 & 683 & 48,768 & 60,955 & - & - & $1,016,437$ & $1,047,298$ \\
\hline Missouri & 2,702 & 2,802 & 46,366 & 44,342 & - & - & $1,059,136$ & $1,174,791$ \\
\hline 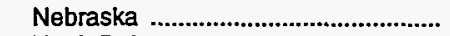 & 396 & 474 & 18,286 & 18,010 & - & - & 643,636 & 722,864 \\
\hline 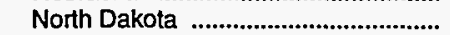 & 1,230 & 1,874 & 8,262 & 6,030 & - & - & 353,124 & 360,800 \\
\hline Ohio & 858 & 771 & 73,489 & 73,893 & - & - & $1,768,769$ & $1,910,309$ \\
\hline 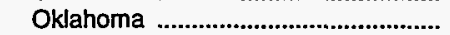 & 1,522 & 1,794 & 21,746 & 33,248 & - & - & 736,444 & 863,812 \\
\hline 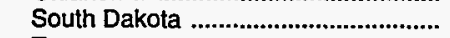 & 888 & 1,711 & 8,931 & 8,587 & - & - & 274,409 & 283,909 \\
\hline Tennessee ................................................... & 1,215 & 1,403 & 65,221 & 62,740 & - & - & $1,130,882$ & $1,163,576$ \\
\hline 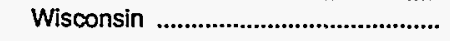 & 2,108 & 3,230 & 38,914 & 61,824 & - & - & $1,035,109$ & $1,083,712$ \\
\hline 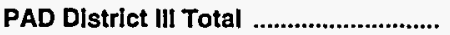 & 191,386 & 186,951 & 432,062 & 472,755 & - & - & $8,151,328$ & $8,901,482$ \\
\hline Alabama & 6,623 & 3,090 & 72,449 & 111,108 & - & - & $1,044,816$ & $1,026,297$ \\
\hline Arkansas ...................................................... & 984 & 1,680 & 38,706 & 26,843 & - & - & 756,912 & 733,524 \\
\hline 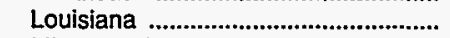 & 31,333 & 40,567 & 83,508 & 118,717 & - & - & $1,620,875$ & $1,862,860$ \\
\hline 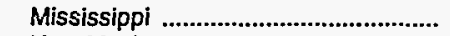 & 5,964 & 4,473 & 38,375 & 45,175 & - & - & 620,903 & 645,913 \\
\hline 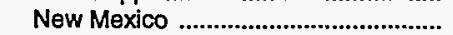 & 1,479 & 2,174 & 17,958 & 20,246 & - & - & 223,032 & 436,426 \\
\hline 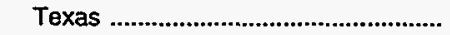 & 145,004 & 134,968 & 181,065 & 150,666 & - & - & $3,884,790$ & $4,196,462$ \\
\hline PAD District IV Total & 1,989 & 4,444 & 116,996 & 131,634 & - & - & $2,050,503$ & $2,075,412$ \\
\hline 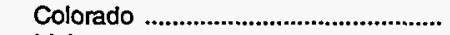 & 1,365 & 855 & 39,006 & 42,468 & - & - & 537,499 & 541,926 \\
\hline 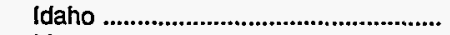 & 76 & 517 & 23,105 & 24,896 & - & - & 334,535 & 349,041 \\
\hline 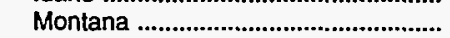 & 331 & 207 & 15,198 & 26,568 & - & - & 353,010 & 348,174 \\
\hline 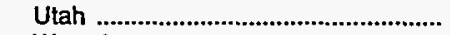 & 201 & 2,846 & 24,326 & 19,652 & - & - & 372,800 & 379,742 \\
\hline 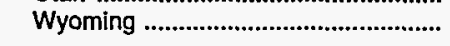 & 14 & 20 & 15,360 & 18,051 & - & - & 452,660 & 456,529 \\
\hline 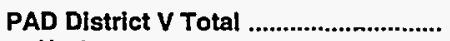 & 153,659 & 159,052 & 415,029 & 367,433 & - & - & $6,781,119$ & $6,843,480$ \\
\hline 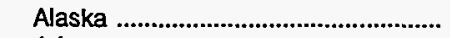 & 5,619 & 5,488 & 40,669 & 33,261 & - & - & 575,014 & 523,670 \\
\hline 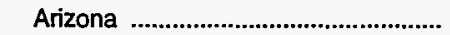 & 996 & 1,078 & 39,407 & 38,988 & - & - & 665,626 & 753,859 \\
\hline California & 128,743 & 94,422 & 170,093 & 164,053 & - & - & $3,225,802$ & $3,200,343$ \\
\hline 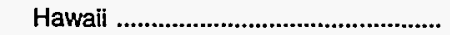 & 8,583 & 19,617 & 13,545 & 7,699 & - & - & 266,648 & 223,041 \\
\hline 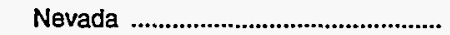 & 1,155 & 1,468 & 14,288 & 18,257 & - & - & 386,778 & 479,765 \\
\hline Oregon & 1,210 & 2,353 & 68,502 & 35,739 & - & - & 730,347 & 699,057 \\
\hline 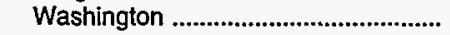 & 7,354 & 34,626 & 68,525 & 69,437 & - & - & 930,904 & 963,745 \\
\hline
\end{tabular}

E Estimated data. Florida's 1996 estimate is based on seven months of data. Nevada's 1996 estimate is based on eight months of data. 11 linois' and Nevada's 1995 data are based on eleven months of data. Oregon's 1995 and Connecticut's 1996 data are a Federal Highway Administration estimate. Dashes $(-)=$ No data reported.

Note: Totals may not equal sum of components due to independent rounding.

Sources: • Energy Information Administration Form EIA-821, "Annual Fuel Oil and Kerosene Sales Report," for 1995 and 1996.

- On-Highway Diesel data are derived from Federal Highway Administation statistics of highway special fuels use. 
Table 5. Sales of Residual Fuel Oil by End Use, 1995 and 1996

(Thousand Gallons)

\begin{tabular}{|c|c|c|c|c|c|c|c|c|}
\hline \multirow{2}{*}{ Destination } & \multicolumn{2}{|c|}{ Commercial } & \multicolumn{2}{|c|}{ Industrial } & \multicolumn{2}{|c|}{ Oil Company } & \multicolumn{2}{|c|}{ Electric Utility } \\
\hline & 1995 & 1996 & 1995 & 1996 & 1995 & 1996 & 1995 & 1996 \\
\hline 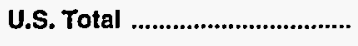 & 922,572 & 927,834 & $1,977,567$ & $2,089,436$ & 211,450 & 167,425 & $3,262,492$ & $4,304,492$ \\
\hline 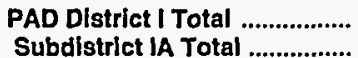 & $\begin{array}{l}896,305 \\
200,493\end{array}$ & $\begin{array}{l}892,897 \\
194,722\end{array}$ & $\begin{array}{r}1,440,632 \\
457,776\end{array}$ & $\begin{array}{r}1,595,527 \\
503,004\end{array}$ & $\begin{array}{r}101,087 \\
207\end{array}$ & $\begin{array}{r}101,783 \\
822\end{array}$ & $\begin{array}{r}2,757,058 \\
636,040\end{array}$ & $\begin{array}{r}3,657,186 \\
940,989\end{array}$ \\
\hline 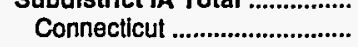 & 18,333 & 19,327 & 30,061 & 40,733 & 207 & 243 & $\begin{array}{r}636,040 \\
91,312\end{array}$ & $\begin{array}{l}940,989 \\
419,126\end{array}$ \\
\hline 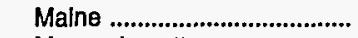 & 15,139 & 21,593 & 302,153 & 328,008 & - & - & 57,674 & 60,487 \\
\hline Massachusetts ........................... & 125,824 & 103,341 & 59,636 & 71,286 & - & 580 & 391,725 & 407,212 \\
\hline Now Hampshire ....................... & 17,864 & 19,010 & 44,753 & 40,680 & - & - & 93,992 & 54,164 \\
\hline 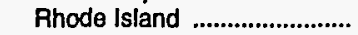 & 20,441 & 28,371 & 15,260 & 13,378 & - & - & 1,338 & - \\
\hline Vermont ..................................... & 2,892 & 3,081 & 5,912 & 8,919 & - & - & - & - \\
\hline Subdlstrict IB Total ..................... & 672,123 & 671,825 & 271,859 & 334,383 & 100,249 & 100,576 & 963,580 & $1,166,608$ \\
\hline 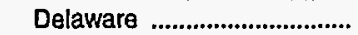 & 5,388 & 9,410 & 28,800 & 39,056 & 35,541 & 23,018 & 76,774 & 68,711 \\
\hline District of Columbia ................ & 5,333 & 4,074 & 6 & 10 & - & - & - & - \\
\hline 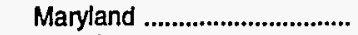 & 4,881 & 4,572 & 29,235 & 57,229 & 630 & 653 & 84,940 & 76,380 \\
\hline 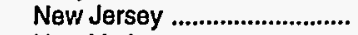 & 50,735 & 54,474 & 75,461 & 68,261 & 2,463 & 2,341 & 83,618 & 143,806 \\
\hline 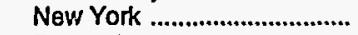 & 555,716 & 543,869 & 80,419 & 103,534 & 1,147 & 893 & 584,682 & 718,952 \\
\hline 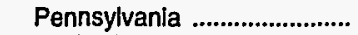 & 50,071 & 55,425 & 57,938 & 66,294 & 60,468 & 73,672 & 133,566 & 158,759 \\
\hline Subdlstrlct IC Total ................... & 23,689 & 26,350 & 710,998 & 758,140 & 631 & 384 & $1,157,438$ & $1,549,590$ \\
\hline Fiorida & 5,649 & 4,198 & 201,259 & 158,621 & 60 & 78 & $1,126,980$ & $1,502,436$ \\
\hline Georgla ......................................... & 471 & 449 & 106,539 & 146,479 & - & - & 1,637 & 4,113 \\
\hline 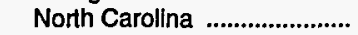 & 7,592 & 9,340 & 236,862 & 266,980 & - & - & - & - \\
\hline 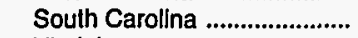 & 1,566 & 1,592 & 86,324 & 95,334 & 168 & 144 & - & - \\
\hline Virginia ........................................ & 8,412 & 10,770 & 71,957 & 75,935 & 402 & 162 & 28,821 & 43,041 \\
\hline West Virginla ................................ & - & - & 8,057 & 14,792 & - & - & - & - \\
\hline PAD District II Total ................. & 14,540 & 22,117 & 202,623 & 255,397 & 64,084 & 28,733 & 86,734 & 97,019 \\
\hline 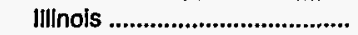 & 1,841 & 8,061 & 4,605 & 21,195 & 10,255 & 3,979 & 41,773 & 45,194 \\
\hline 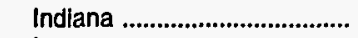 & 1,301 & 577 & 34,947 & 43,439 & 29,284 & - & 3,574 & - \\
\hline 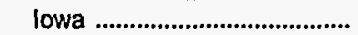 & - & 34 & 3,769 & 3,963 & - & - & - & - \\
\hline 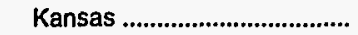 & 483 & 76 & 752 & 5,637 & - & - & - & - \\
\hline Kentucky .................................... & - & 7 & 3,540 & 6,647 & 4,709 & 3,681 & - & - \\
\hline 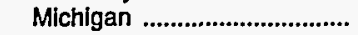 & 197 & 194 & 15,472 & 15,022 & 956 & 2,610 & 33,394 & 39,797 \\
\hline Minnesota ................................... & 4,570 & 5,881 & 13,334 & 18,387 & 8,623 & 8,959 & - & 3,417 \\
\hline Mlssouri ……………………............ & 42 & 240 & 13,085 & 13,120 & - & - & 6,818 & 8,004 \\
\hline 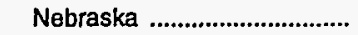 & 25 & - & 4,925 & 7,114 & - & - & - & - \\
\hline North Dakola .............................. & 775 & 247 & 2,593 & 5,504 & 3,349 & - & - & 5 \\
\hline Ohlo & 186 & 73 & 52,703 & 63,305 & 3,114 & 4,726 & 1,175 & 602 \\
\hline 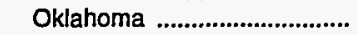 & 11 & - & 13,490 & 10,994 & - & - & - & - \\
\hline 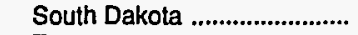 & 100 & - & 460 & 1,700 & - & - & - & - \\
\hline 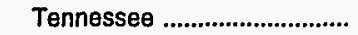 & 582 & 1,178 & 14,001 & 7,566 & 178 & 113 & - & - \\
\hline 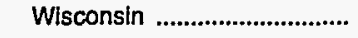 & 4,429 & 5,550 & 24,947 & 31,801 & 3,617 & 4,665 & - & - \\
\hline PAD Dlstrict III Total ................. & 108 & 59 & 155,920 & 168,522 & 192 & - & 463 & 79,902 \\
\hline Alabama & 108 & 24 & 20,653 & 29,974 & - & - & - & - \\
\hline 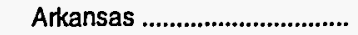 & - & 9 & 8,362 & 4,915 & - & - & - & 4,849 \\
\hline 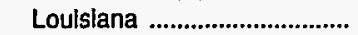 & - & 26 & 15,659 & 31,670 & - & - & 435 & 5,675 \\
\hline 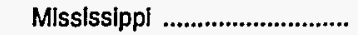 & - & - & 3,303 & 4,778 & - & - & 27 & 69,336 \\
\hline 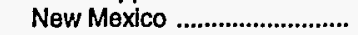 & - & - & 7,323 & 8,239 & - & - & - & - \\
\hline 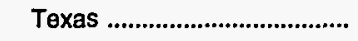 & - & - & 100,619 & 88,946 & 192 & - & - & 43 \\
\hline PAD Dlstrict IV Total ................. & 848 & 865 & 10,055 & 3,659 & 11,779 & 7,488 & 311 & - \\
\hline 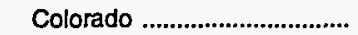 & - & - & 7 & 168 & - & - & - & - \\
\hline 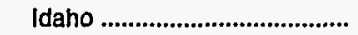 & 178 & 179 & 93 & 96 & - & - & 245 & - \\
\hline 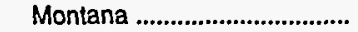 & 135 & 106 & 841 & 99 & 8,707 & 7,488 & - & - \\
\hline Utah ................................................ & 523 & 574 & 8,434 & 3,087 & 3,073 & - & - & - \\
\hline 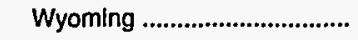 & 12 & 6 & 680 & 209 & - & - & 66 & - \\
\hline PAD District $V$ Total ..................... & 10,770 & 11,896 & 168,338 & 66,332 & 34,309 & 29,421 & 417,927 & 470,384 \\
\hline 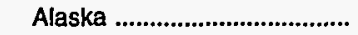 & - & - & 15,377 & 16,456 & - & - & - & - \\
\hline 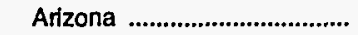 & - & 204 & 2,782 & 3,382 & 42 & - & - & - \\
\hline Calffomia ..................................... & 164 & 520 & 59,985 & 11,478 & 36 & 1,427 & - & - \\
\hline Hawall ............................................. & 2,554 & 535 & 7,354 & 10,858 & 32,992 & 27,256 & 417,822 & 470,307 \\
\hline Nevada ........................................... & - & - & 44,324 & 5,368 & 40 & 114 & - & - \\
\hline Oregon & 3,554 & 3,511 & 13,303 & 5,680 & - & - & 105 & 77 \\
\hline 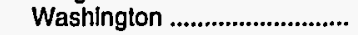 & 4,499 & 7,125 & 25,213 & 13,109 & 1,199 & 624 & - & - \\
\hline
\end{tabular}

See footnotes at end of table. 
Table 5. Sales of Residual Fuel Oil by End Use, 1995 and 1996 (Continued)

(Thousand Gallons)

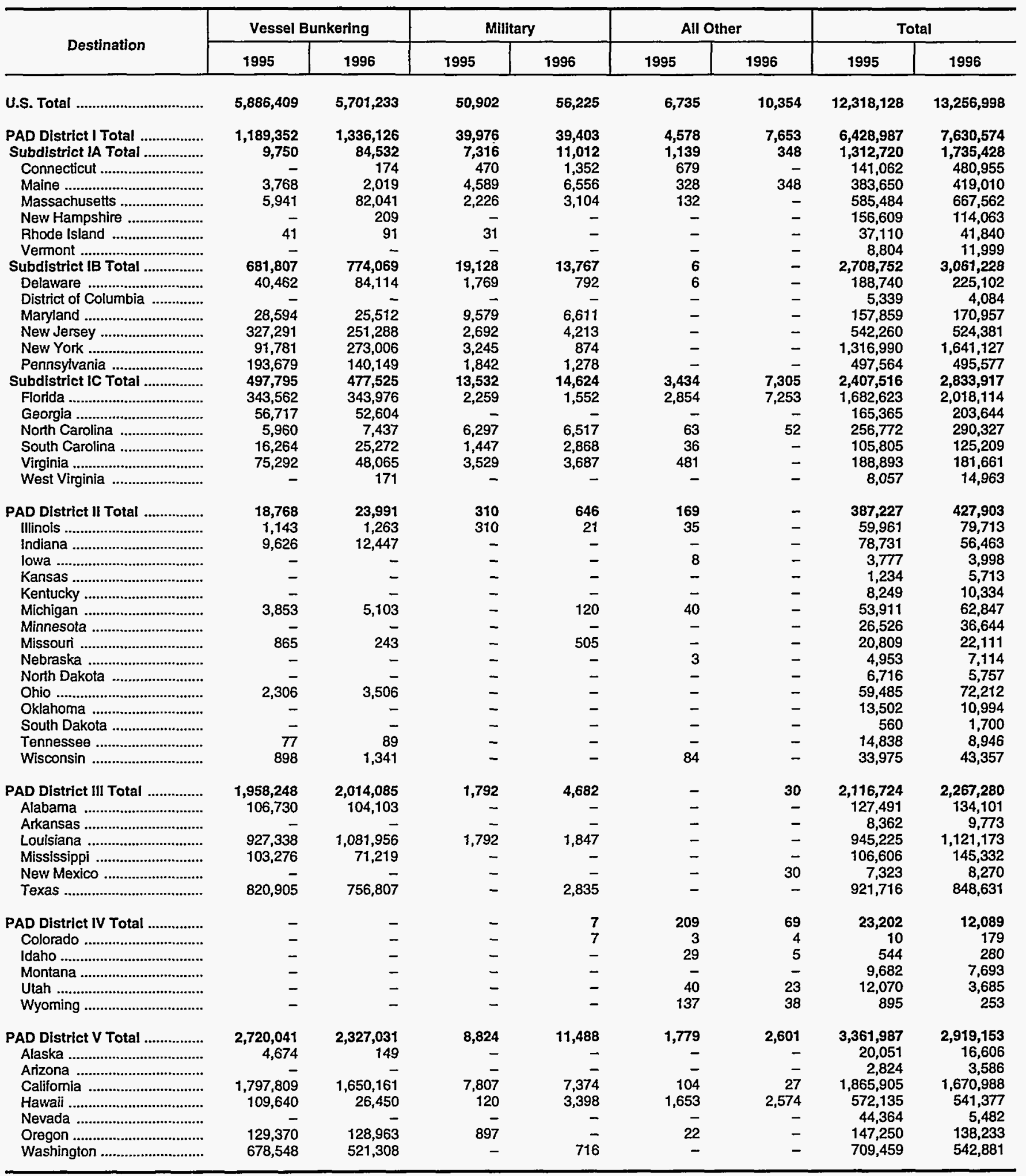

Dashes $(-)=$ No data reported.

Note: Totals may not equal sum of components due to independent rounding

Sources: - Energy Information Administration Form EiA-821, "Annual Fuel Oil and Kerosene Sales Report," for 1995 and 1996. 
Table 6. Sales of Kerosene by End Use, 1995 and 1996

(Thousand Gallons)

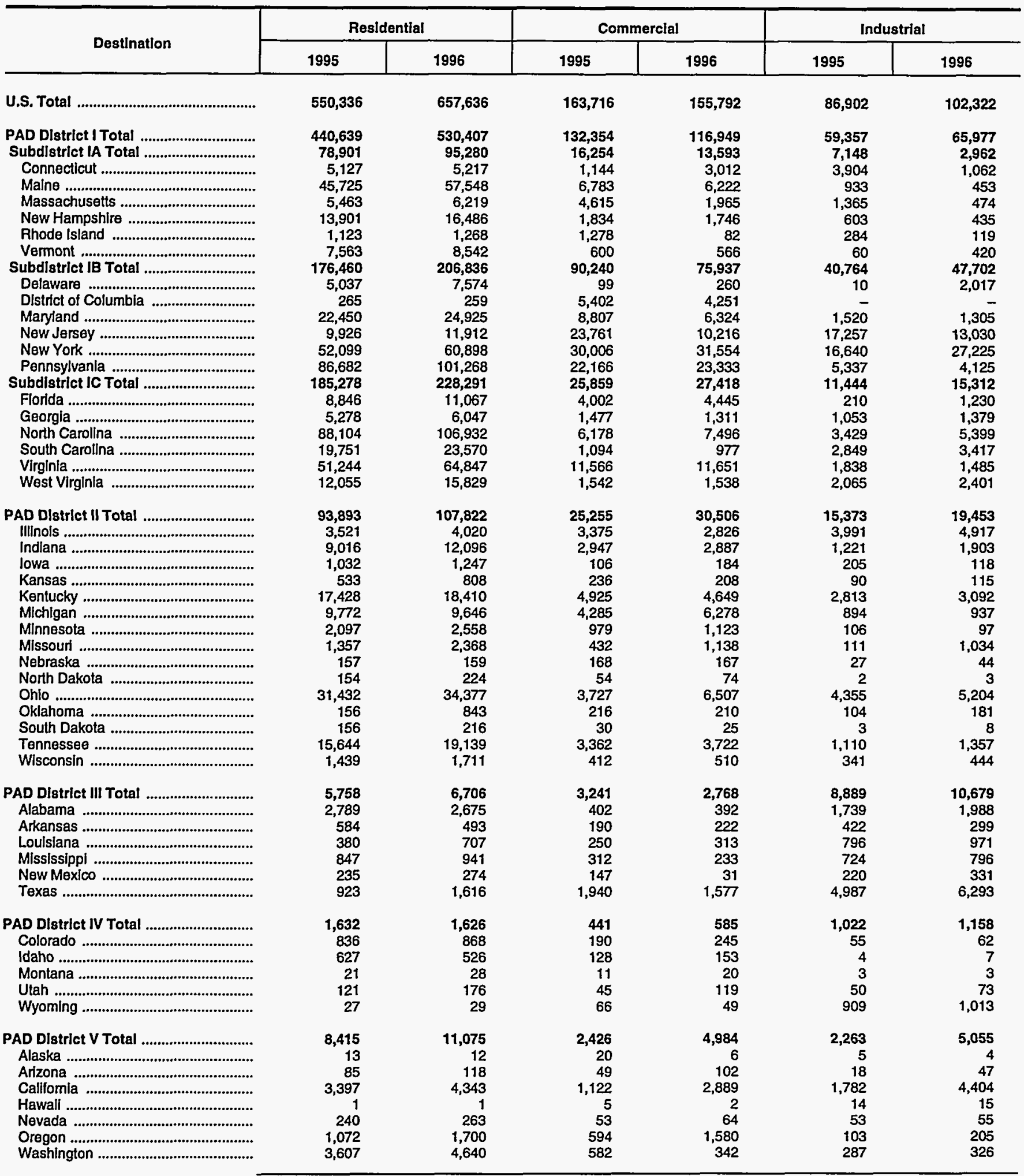

See footnotes at end of table. 
Table 6. Sales of Kerosene by End Use, 1995 and 1996 (Continued)

(Thousand Gallons)

\begin{tabular}{|c|c|c|c|c|c|c|}
\hline \multirow{2}{*}{ Destination } & \multicolumn{2}{|c|}{ Farm } & \multicolumn{2}{|c|}{ All Other } & \multicolumn{2}{|c|}{ Total } \\
\hline & 1995 & 1996 & 1995 & 1996 & 1995 & 1996 \\
\hline 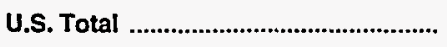 & 19,919 & 26,017 & 7,576 & 7,223 & 828,450 & 948,990 \\
\hline 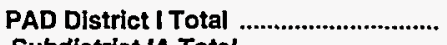 & 7,629 & 9,408 & 3,476 & 3,008 & 643,455 & 725,749 \\
\hline 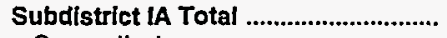 & 685 & 974 & 413 & 236 & 103,401 & 113,046 \\
\hline 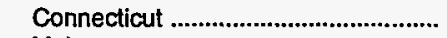 & 20 & 9 & 58 & - & 10,254 & 9,300 \\
\hline Maine & 220 & 239 & 146 & 40 & 53,806 & 64,502 \\
\hline 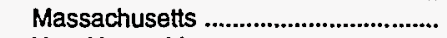 & 65 & 106 & 51 & 26 & 11,559 & 8,788 \\
\hline New Hampshire . .................................... & 105 & 186 & 86 & 81 & 16,529 & 18,934 \\
\hline 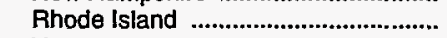 & - & - & - & 1 & 2,685 & 1,470 \\
\hline 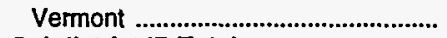 & 274 & 434 & 72 & 88 & 8,569 & 10,050 \\
\hline 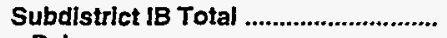 & 1,707 & 2,702 & 1,800 & 2,244 & 310,972 & 335,421 \\
\hline 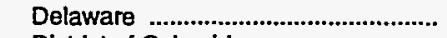 & 44 & 35 & 151 & 1 & 5,341 & 9,886 \\
\hline 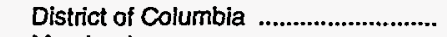 & - & - & - & - & 5,667 & 4,510 \\
\hline 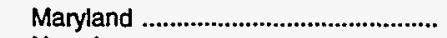 & 728 & 875 & 157 & 265 & 33,662 & 33,693 \\
\hline 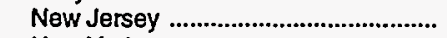 & 7 & 38 & 126 & 140 & 51,077 & 35,337 \\
\hline 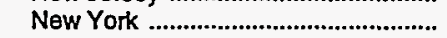 & 287 & 1,099 & 255 & 337 & 99,287 & 121,113 \\
\hline 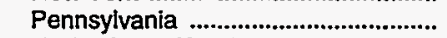 & 641 & 655 & 1,111 & 1,501 & 115,938 & 130,882 \\
\hline 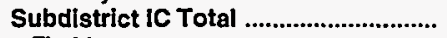 & 5,238 & 5,732 & 1,262 & 528 & 229,082 & 277,282 \\
\hline Florida & 80 & 140 & 18 & 8 & 13,156 & 16,891 \\
\hline Georgia & 172 & 2 & 227 & 159 & 8,206 & 8,898 \\
\hline North Carolina & 781 & 1,433 & 619 & 105 & 99,111 & 121,364 \\
\hline South Carolina .................................... & 217 & 279 & 182 & 11 & 24,094 & 28,254 \\
\hline 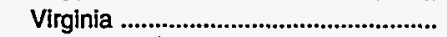 & 3,246 & 3,239 & 56 & 59 & 67,951 & 81,282 \\
\hline West Virginia & 743 & 639 & 160 & 186 & 16,564 & 20,593 \\
\hline 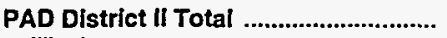 & 10,186 & 14,878 & 2,603 & 2,916 & 147,310 & 175,575 \\
\hline 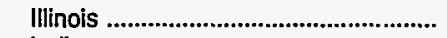 & 881 & 4,303 & 547 & 666 & 12,315 & 16,733 \\
\hline 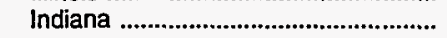 & 498 & 1,331 & 182 & 296 & 13,865 & 18,513 \\
\hline lowa & 1,499 & 691 & 37 & 23 & 2,879 & 2,262 \\
\hline 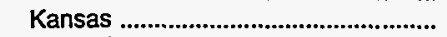 & 222 & 397 & 115 & 21 & 1,197 & 1,549 \\
\hline 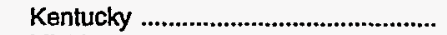 & 1,305 & 1,729 & 724 & 273 & 27,194 & 28,152 \\
\hline 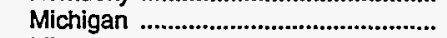 & 233 & 382 & 205 & 429 & 15,389 & 17,672 \\
\hline Minnesota & 1,167 & 1,190 & 14 & 191 & 4,362 & 5,158 \\
\hline 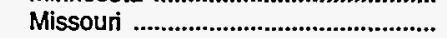 & 237 & 302 & 95 & 49 & 2,231 & 4,892 \\
\hline 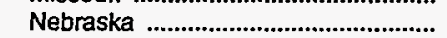 & 249 & 315 & 101 & 125 & 702 & 810 \\
\hline North Dakota & 8 & 7 & 6 & 15 & 224 & 322 \\
\hline Ohio & 3,161 & 3,488 & 350 & 592 & 43,025 & 50,167 \\
\hline 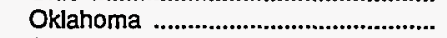 & 82 & 62 & 65 & 55 & 623 & 1,351 \\
\hline 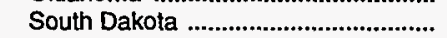 & 67 & 99 & 3 & 15 & 259 & 364 \\
\hline 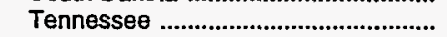 & 374 & 264 & 74 & 104 & 20,564 & 24,586 \\
\hline 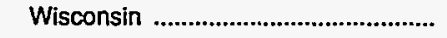 & 204 & 318 & 84 & 62 & 2,480 & 3,046 \\
\hline 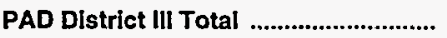 & 425 & 516 & 837 & 517 & 19,149 & 21,185 \\
\hline 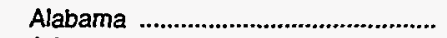 & 9 & 8 & 135 & 19 & 5,075 & 5,082 \\
\hline 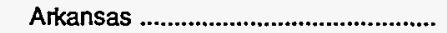 & 84 & 52 & 349 & 34 & 1,629 & 1,099 \\
\hline 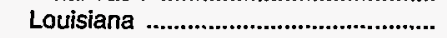 & 17 & 69 & 111 & 214 & 1,554 & 2,274 \\
\hline 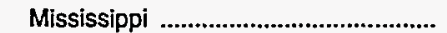 & 24 & 84 & 67 & 9 & 1,974 & 2,064 \\
\hline 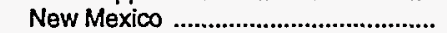 & 24 & 13 & 52 & 59 & 678 & 709 \\
\hline 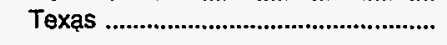 & 267 & 290 & 122 & 182 & 8,240 & 9,958 \\
\hline PAD District IV Total .............................. & 175 & 186 & 101 & 126 & 3,369 & 3,681 \\
\hline 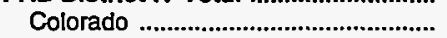 & 84 & 120 & 58 & 71 & 1,223 & 1,366 \\
\hline Idaho & 35 & 35 & 34 & 5 & 828 & 726 \\
\hline Montana & 4 & 3 & - & 1 & 40 & 54 \\
\hline Utah & 52 & 20 & 2 & 7 & 270 & 394 \\
\hline 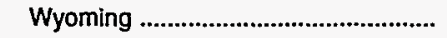 & - & 9 & 6 & 41 & 1,008 & 1,141 \\
\hline 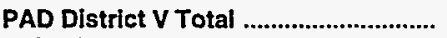 & 1,504 & 1,029 & 560 & 657 & 15,167 & 22,801 \\
\hline 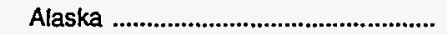 & - & - & - & 1 & 37 & 23 \\
\hline Arizona & 4 & 5 & 2 & 17 & 159 & 288 \\
\hline California & 308 & 359 & 267 & 365 & 6,877 & 12,360 \\
\hline 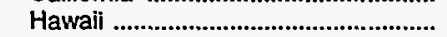 & 1 & 3 & - & - & 22 & 22 \\
\hline Nevada & 12 & 15 & 11 & 3 & 370 & 399 \\
\hline 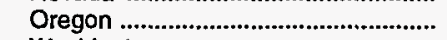 & 605 & 71 & 248 & 182 & 2,623 & 3,737 \\
\hline 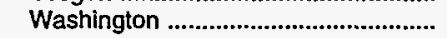 & 574 & 576 & 31 & 89 & 5,081 & 5,972 \\
\hline
\end{tabular}

Dashes $(-)=$ No data reported.

Note: Totals may not equal sum of components due to independent rounding.

Sources: - Energy Information Administration Form ElA-821, "Annual Fuel Oil and Kerosene Sales Report," for 1995 and 1996. 
Table 7. Sales for Residential Use: Distillate Fuel Oil and Kerosene, 1996 (Thousand Gallons)

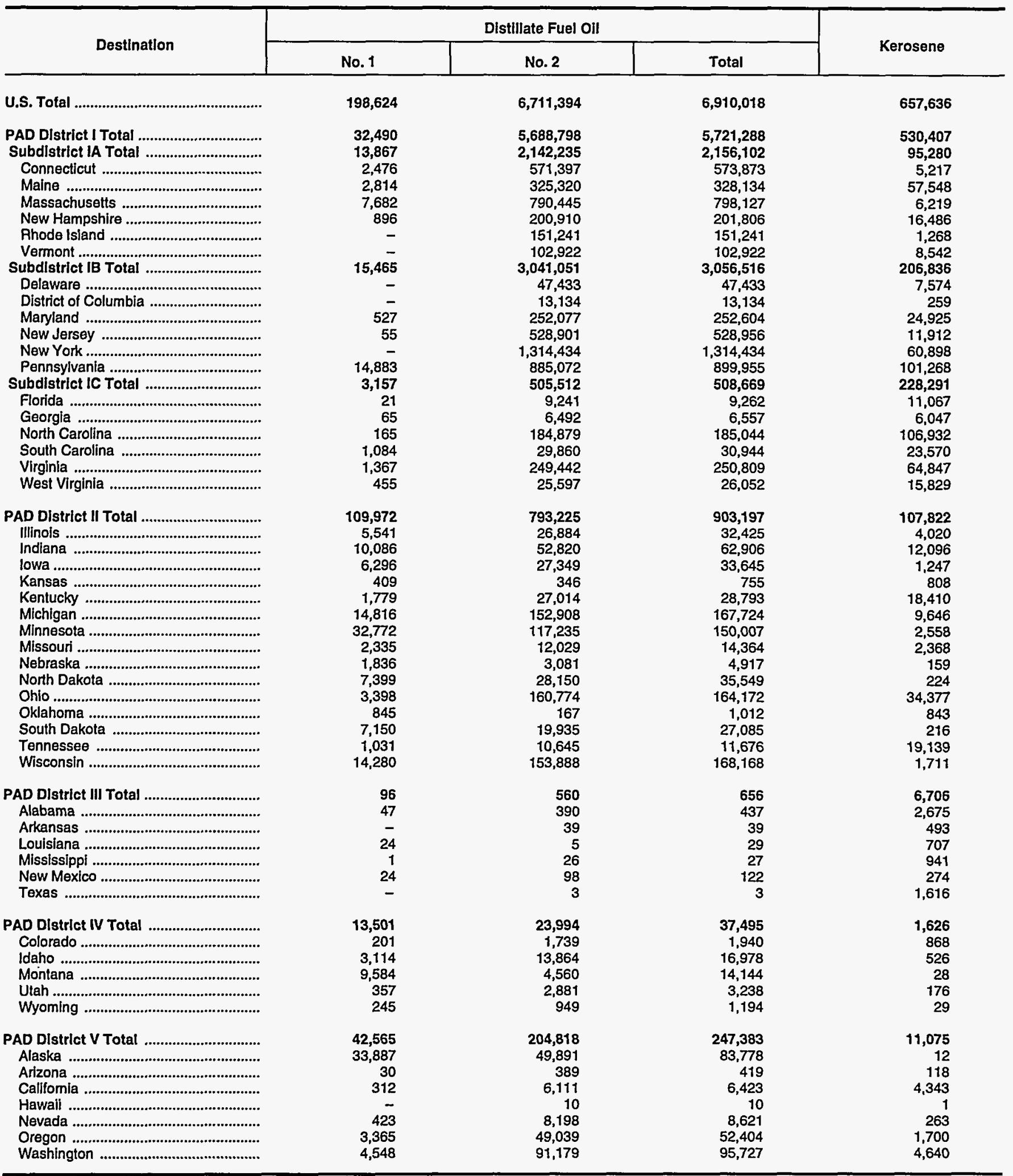

Dashes $(-)=$ No data reported.

Note: Totals may not equal sum of components due to independent rounding.

Sources: - Energy Information Administration Form ElA-821, "Annual Fuel Oil and Kerosene Sales Report," for 1996.

- Kerosene data are based on data from the Energy Information Administration's "Petroleum Supply Annual," Volume 1, 1996, (DOE/EIA-0340(96)/1). 
Table 8. Sales for Commercial Use: Distillate Fuel Oil, Residual Fuel Oil, and Kerosene, 1996 (Thousand Gallons)

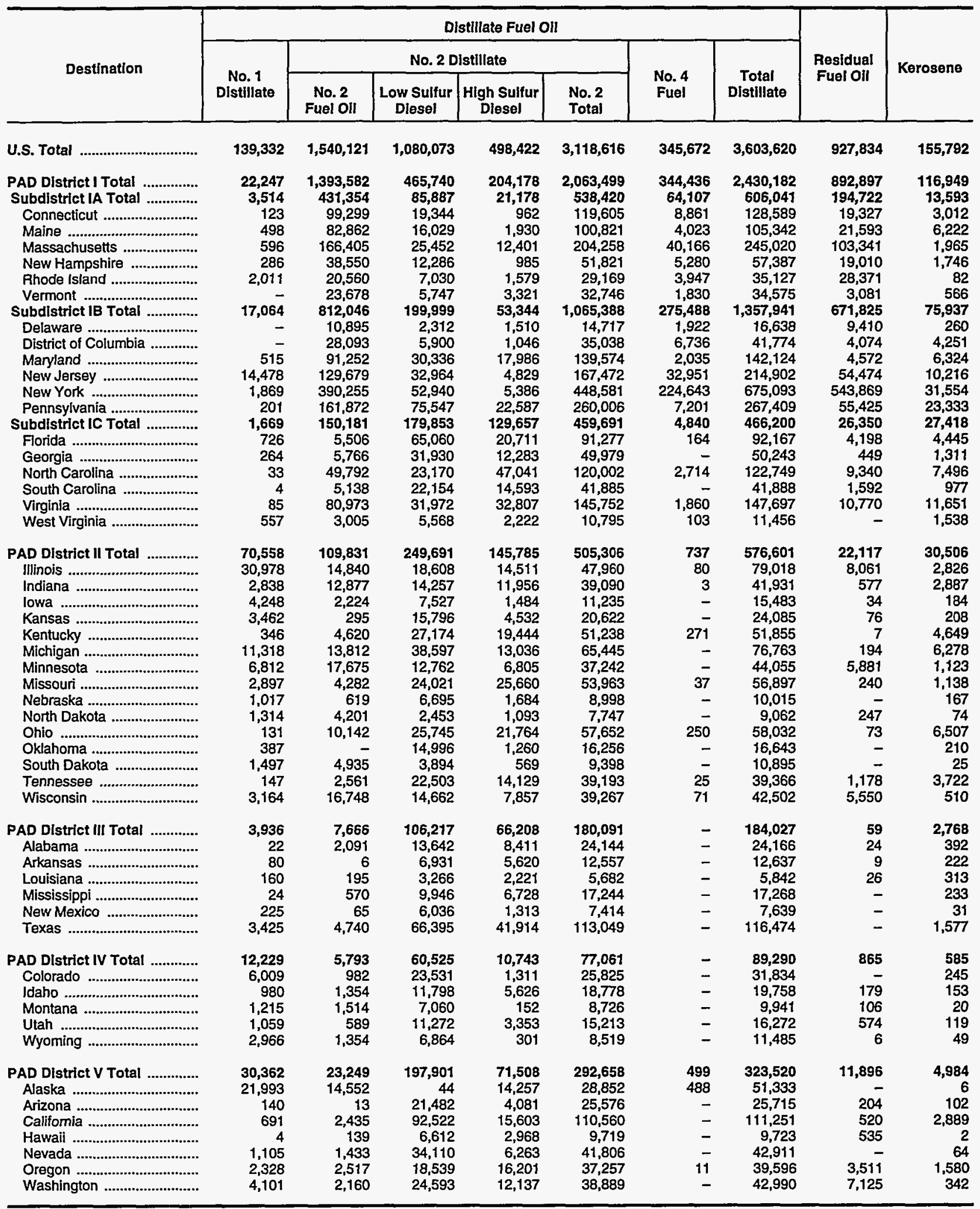

Dashes $(-)=$ No data reported.

Note: Totals may not equal sum of components due to independent rounding.

Sources; - Energy Information Administration Form ElA-821, "Annual Fuel Oil and Kerosene Sales Report," for 1996.

- Kerosene data are based on data from the Energy Information Administration's "Petroleum Supply Annual," Volume 1, 1996, (DOE/EIA-0340(96)/1). 
Table 9. Sales for Industrial Use: Distillate Fuel Oil, Residual Fuel Oil, and Kerosene, 1996 (Thousand Gallons)

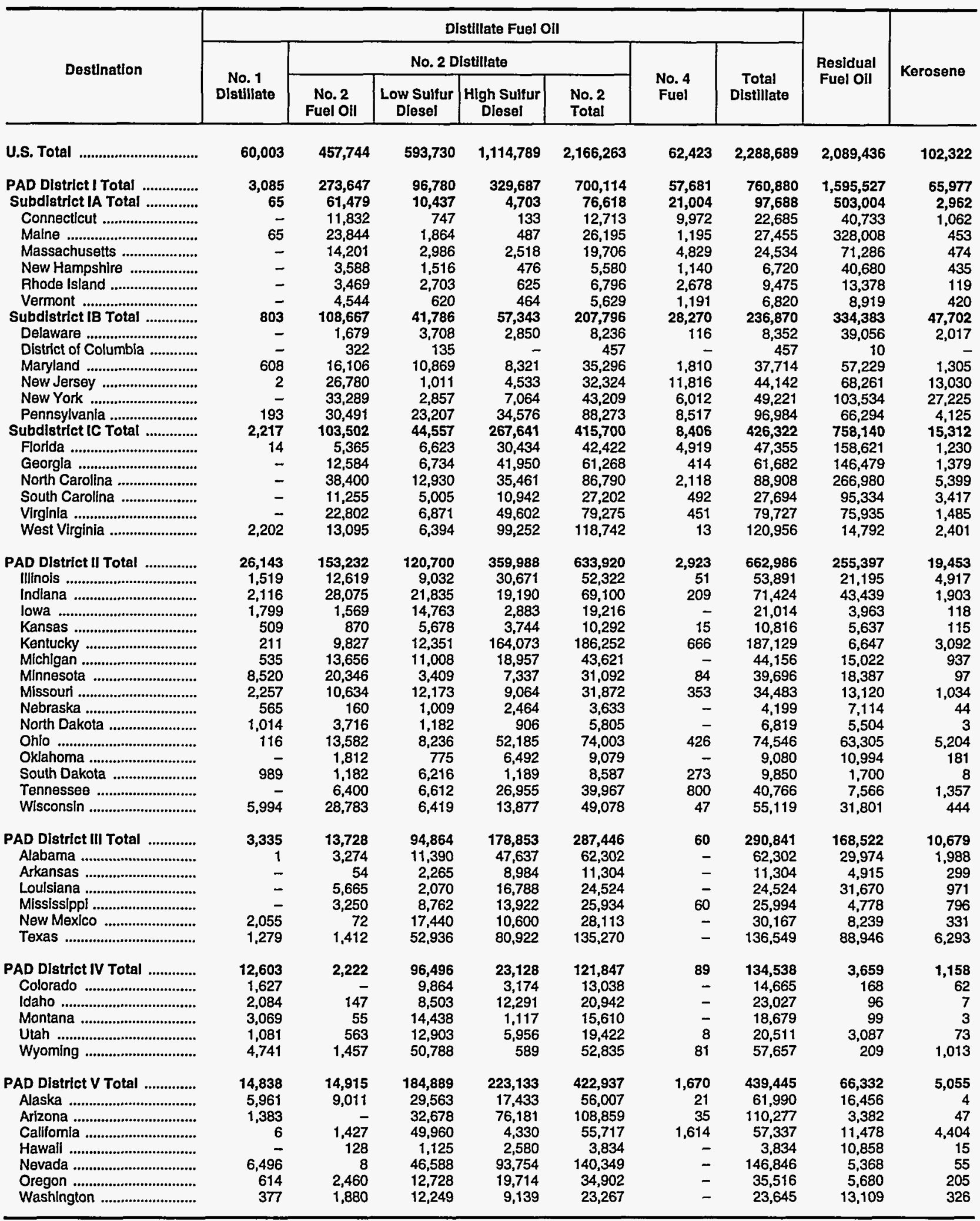

Dashes $(-)=$ No data reported.

Note: Totals may not equal sum of components due to independent rounding.

Sources: - Energy Information Administration Form ElA-821, "Annual Fuel Oil and Kerosene Sales Report." for 1996.

- Kerosene data are based on data from the Energy Information Administration's "Petroleum Supply Annual," Volume 1, 1996, (DOE/EIA-0340(96)/1). 
Table 10. Sales for Farm Use: Distillate Fuel Oil and Kerosene; Sales for Electric Utility and Oil Company Uses: Distillate Fuel Oil and Residual Fuel Oil, 1996 (Thousand Gallons)

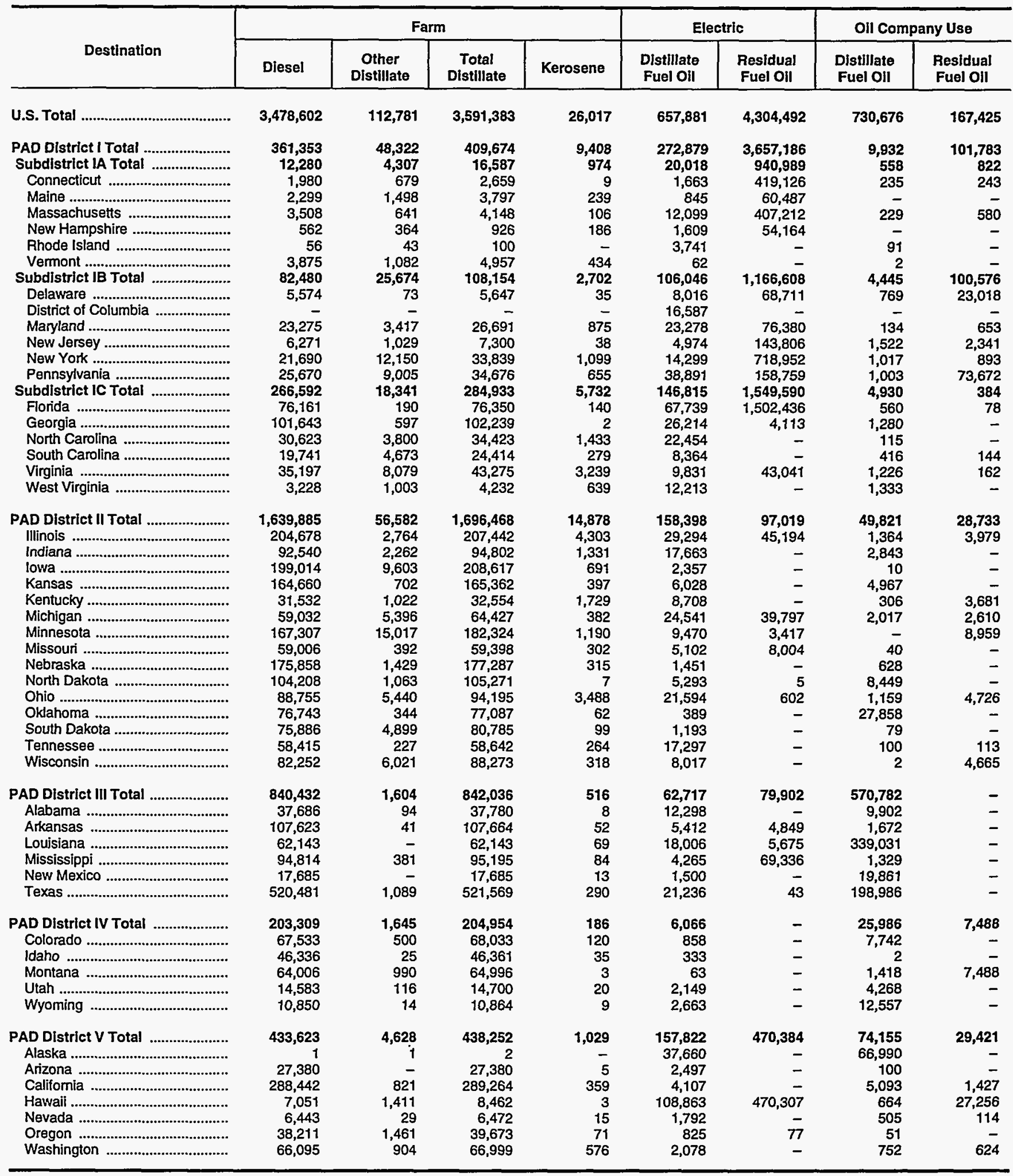

Dashes $(-)=$ No data reported

Note: Totals may not equal sum of components due to independent rounding

Sources: - Energy Information Administration Form ElA-821, "Annual Fuel Oil and Kerosene Sales Report," for 1996.

- Kerosene data are based on data from the Energy Information Administration's "Petroleum Supply Annual," Volume 1, 1996, (DOE/EIA-0340(96)/1). 
Table 11. Sales for Transportation Use: Distillate Fuel Oil and Residual Fuel Oil, 1996 (Thousand Gallons)

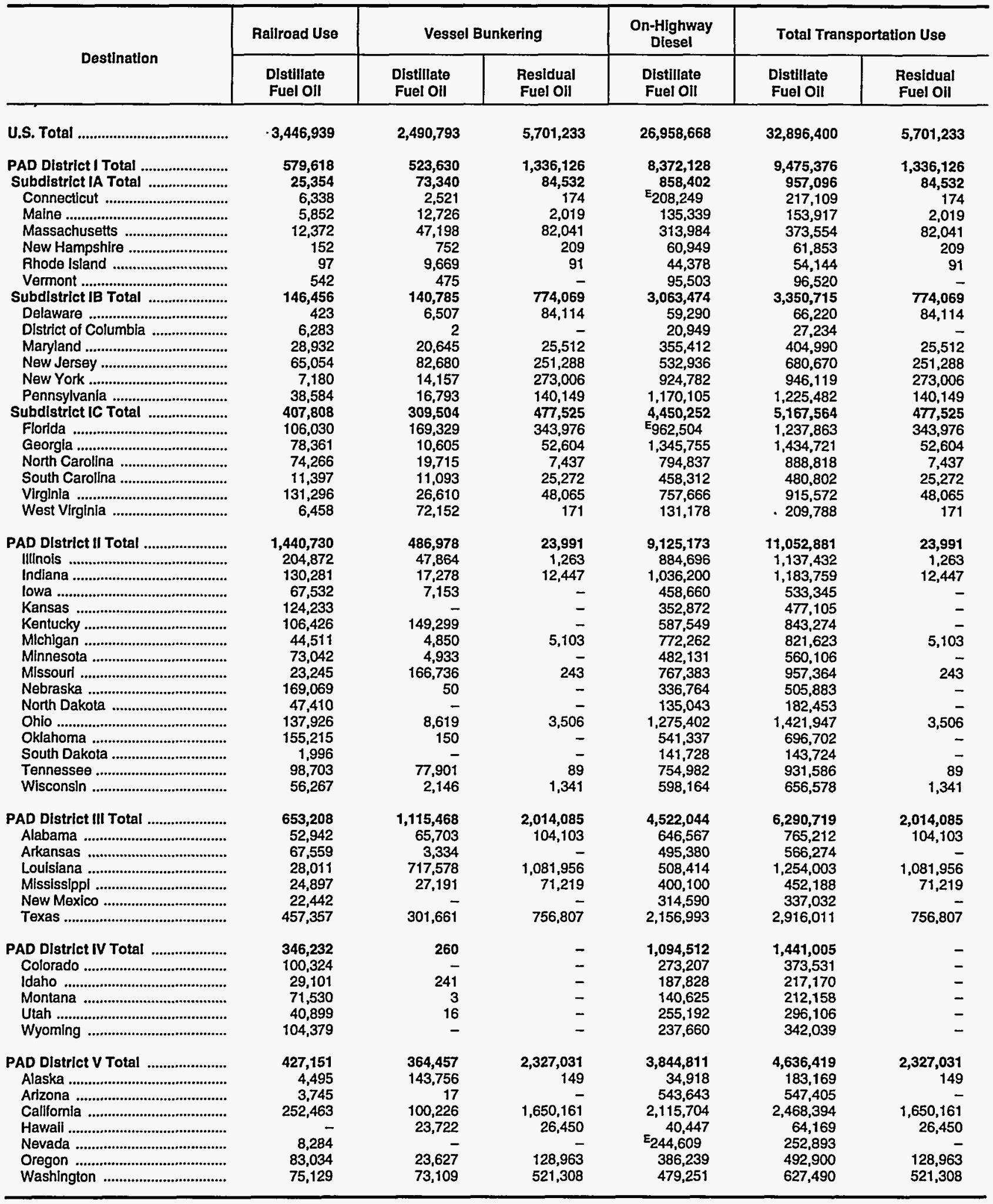

E Estimated data. Forida's 1996 estimate is based on seven months of data. Nevada's 1996 estimate is based on eight months of data. Connecticut's 1996 data are a Federal Highway Administration estimate.

Dashes $(-)=$ No data reported.

Note: Totals may not equal sum of components due to independent rounding.

Sources: • Energy Information Administration Form EIA-821. "Annual Fuel Oil and Kerosene Sales Report," for 1996.

- On-Highway Dlesel data are Federal Highway Administation statistics of highway special fuels use. 
Table 12. Sales for Military, Off-Highway, and All Other Uses: Distillate Fuel Oil, Residual Fuel Oil, and Kerosene, 1996

(Thousand Gallons)

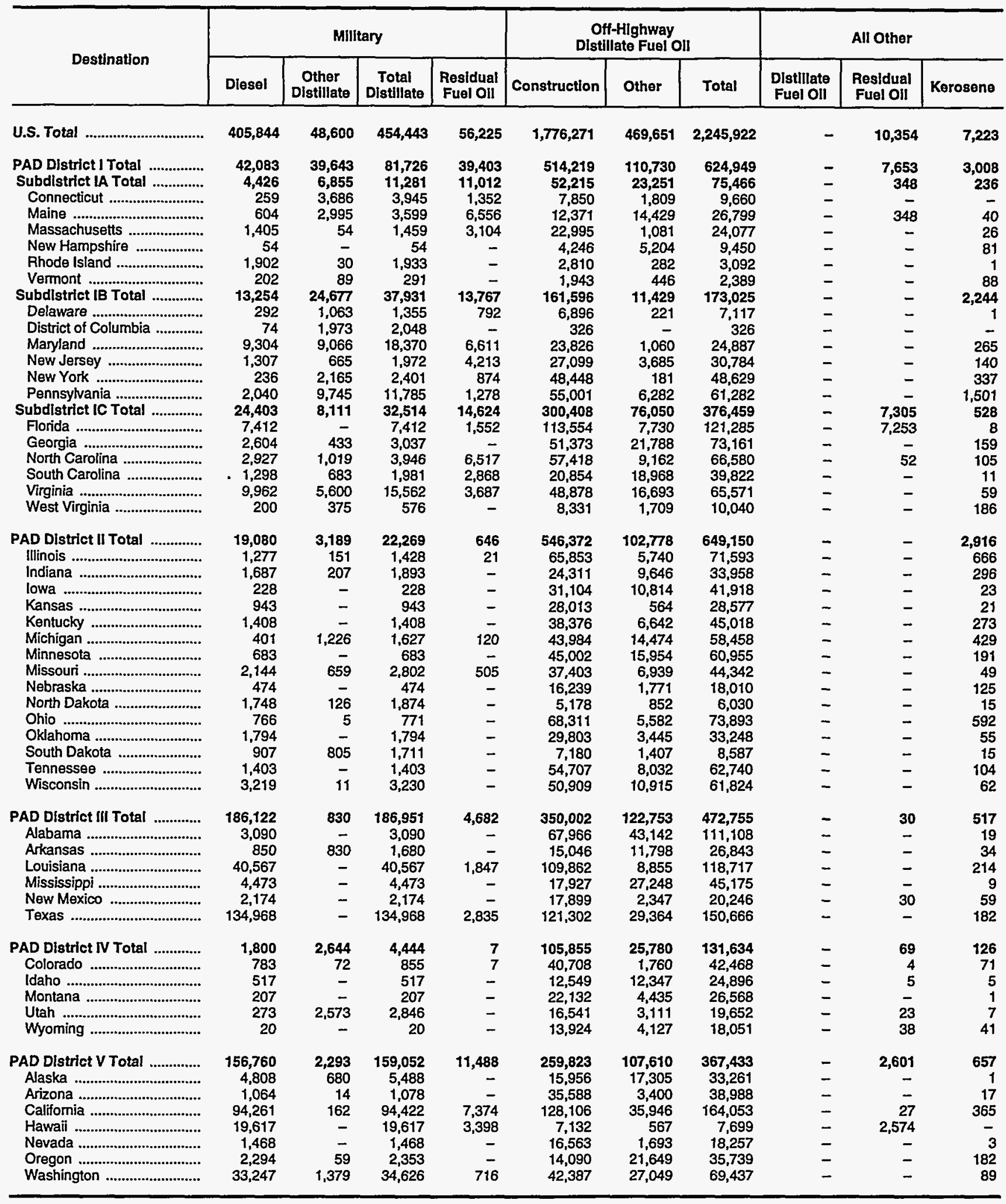

Dashes $(-)=$ No data reported.

Note: Totals may not equal sum of components due to independent rounding.

Sources: - Energy Information Administration Form EIA-821, "Annual Fuel Oil and Kerosene Sales Report," for 1996.

- Kerosene data are based on data from the Energy Information Administration's "Petroleum Supply Annual," Volume 1, 1996, (DOE/EIA-0340(96)/1). 


\section{Adjusted Sales of Fuel Oil and Kerosene in 1996}

The following tables contain estimates of distillate fuel oil that have been adjusted at the PAD district level to equal published EIA volume estimates of petroleum products supplied in the U.S. marketplace. The kerosene and residual fuel oil sales estimates have been adjusted at the national level. Historically, kerosene estimates have always been adjusted at the PAD district level. However, in 1992, certain kerosene estimates at the PAD district level resulted in negative numbers. Hence, the adjustment procedure was changed to display positive sales volumes of kerosene for all $\mathrm{PAD}$ districts.
The products supplied estimates can be found in the 1994 through 1996 issues of the Petroleum Supply Annual. For certain sales categories, data obtained from alternate sources are used instead of the adjusted numbers. See "Technical Note 3" in the Appendix for further explanation of data adjustments.

Total domestic adjusted sales of fuel oil and kerosene increased 4.2 percent from 63.0 billion gallons in 1995 to 65.7 billion gallons in 1996 . While adjusted sales of distillate increased 5.2 percent in 1996, residual fuel oil dropped 0.1 percent. Kerosene adjusted sales increased by 14.6 percent.

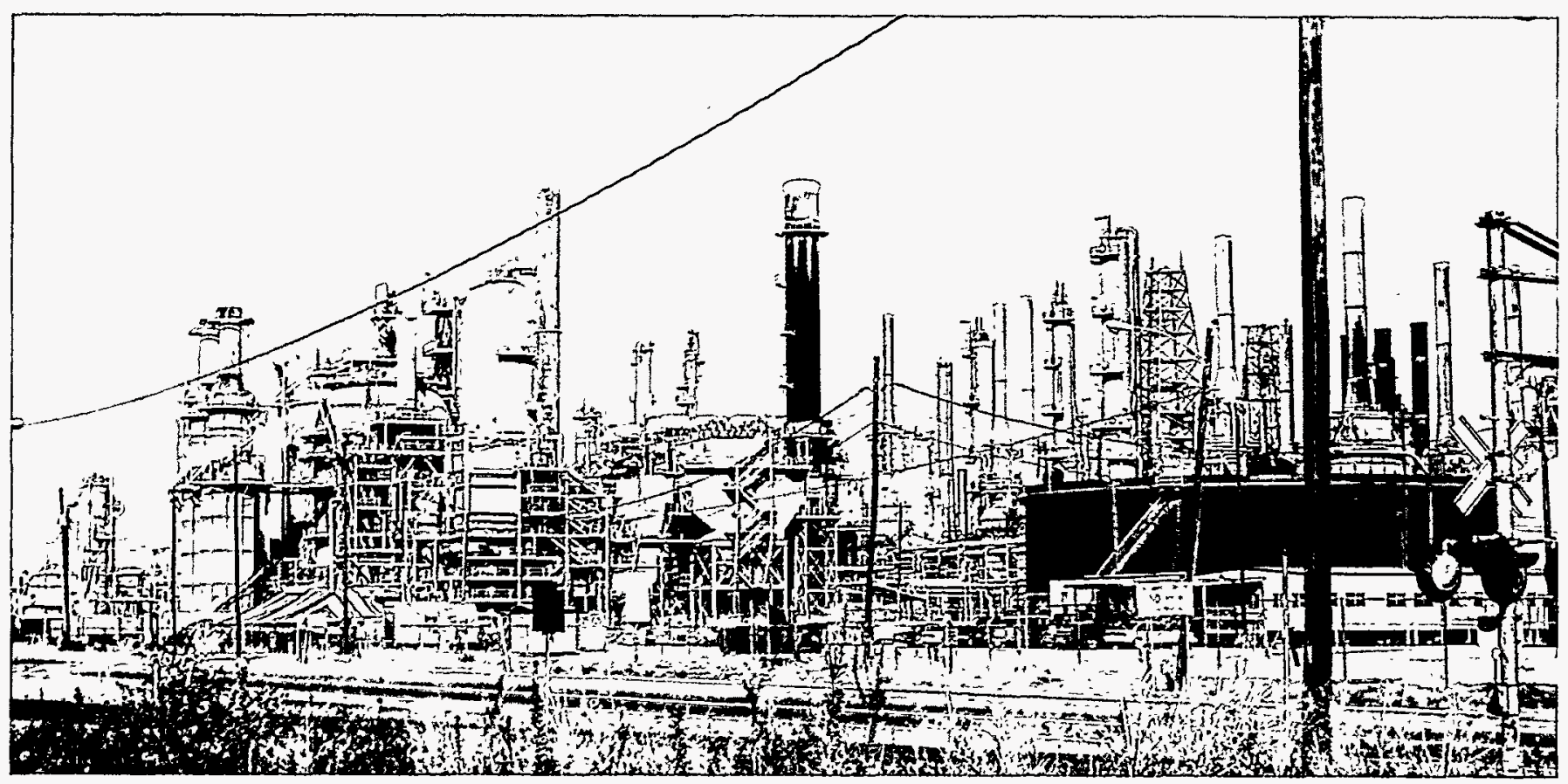

Sales to chemical plants are classified as industrial use. 
Table 13. Adjusted Sales of Distillate Fuel Oil by End Use in the United States: 1992-1996 (Thousand Gallons)

\begin{tabular}{|c|c|c|c|c|c|}
\hline \multirow{2}{*}{ End Use } & \multicolumn{5}{|c|}{ Distillate Fuel Oll } \\
\hline & 1992 & 1993 & 1994 & 1995 & 1996 \\
\hline U.S. Total & $45,791,466$ & $46,621,806$ & $48,477,156$ & $49,157,598$ & $51,730,518$ \\
\hline 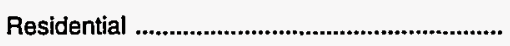 & $6,238,133$ & $6,577,146$ & $6,335,853$ & $6,370,381$ & $6,707,714$ \\
\hline 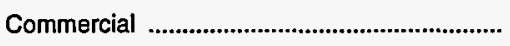 & $3,347,133$ & $3,342,105$ & $3,343,090$ & $3,318,818$ & $3,433,555$ \\
\hline Industrial & $2,218,843$ & $1,965,557$ & $2,091,611$ & $2,027,409$ & $2,107,190$ \\
\hline$\ldots+\ldots$ & 785,120 & 763,068 & 703,946 & 556,126 & 625,608 \\
\hline Farm & $3,504,846$ & $3,238,675$ & $3,198,640$ & $3,230,765$ & $3,334,420$ \\
\hline (n) & 468,795 & 586,845 & 755,219 & 601,185 & 684,263 \\
\hline Railroad & $3,217,231$ & $2,906,998$ & $3,063,441$ & $3,191,023$ & $3,266,861$ \\
\hline Vessel Bunkering & $2,240,170$ & $2,043,745$ & $2,026,899$ & $1,978,105$ & $2,177,608$ \\
\hline On-Highway Diesel & $21,374,872$ & $22,758,842$ & $24,442,609$ & $25,576,433$ & $26,958,668$ \\
\hline Military & 650,178 & 482,773 & 526,563 & 368,219 & 371,093 \\
\hline 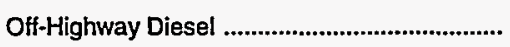 & $1,739,068$ & $1,953,640$ & $1,988,481$ & $1,939,133$ & $2,063,538$ \\
\hline All Other & 7.075 & 2,411 & 804 & - & - \\
\hline
\end{tabular}

Dashes $(-)=$ No data reported.

Note: Totals may not equal sum of components due to independent rounding.

Note: Distillate fuel oil data in the Adjusted Sales tables (13-24) are adjusted at the PAD District level. See Technical Note 3 for further explanation.

Sources: - Energy Information Administration Form ElA-821, "Annual Fuel Oil and Kerosene Sales Report," for 1992-1996.

- Electric Utility data are based on data obtained on Form ElA-759, "Monthly Power Plant Report," and Form FERC-423, "Monthly Report of Cost and Quality of Fuels for Electric Plants."

- On-Highway Diesel data are. Federal Highway Administation statistics of highway special fuels use.

- Energy Information Administration's "Petroleum Supply Annual," Volume 1, 1992-1996, (DOE/ElA-0340(92)/1 - (96)/1).

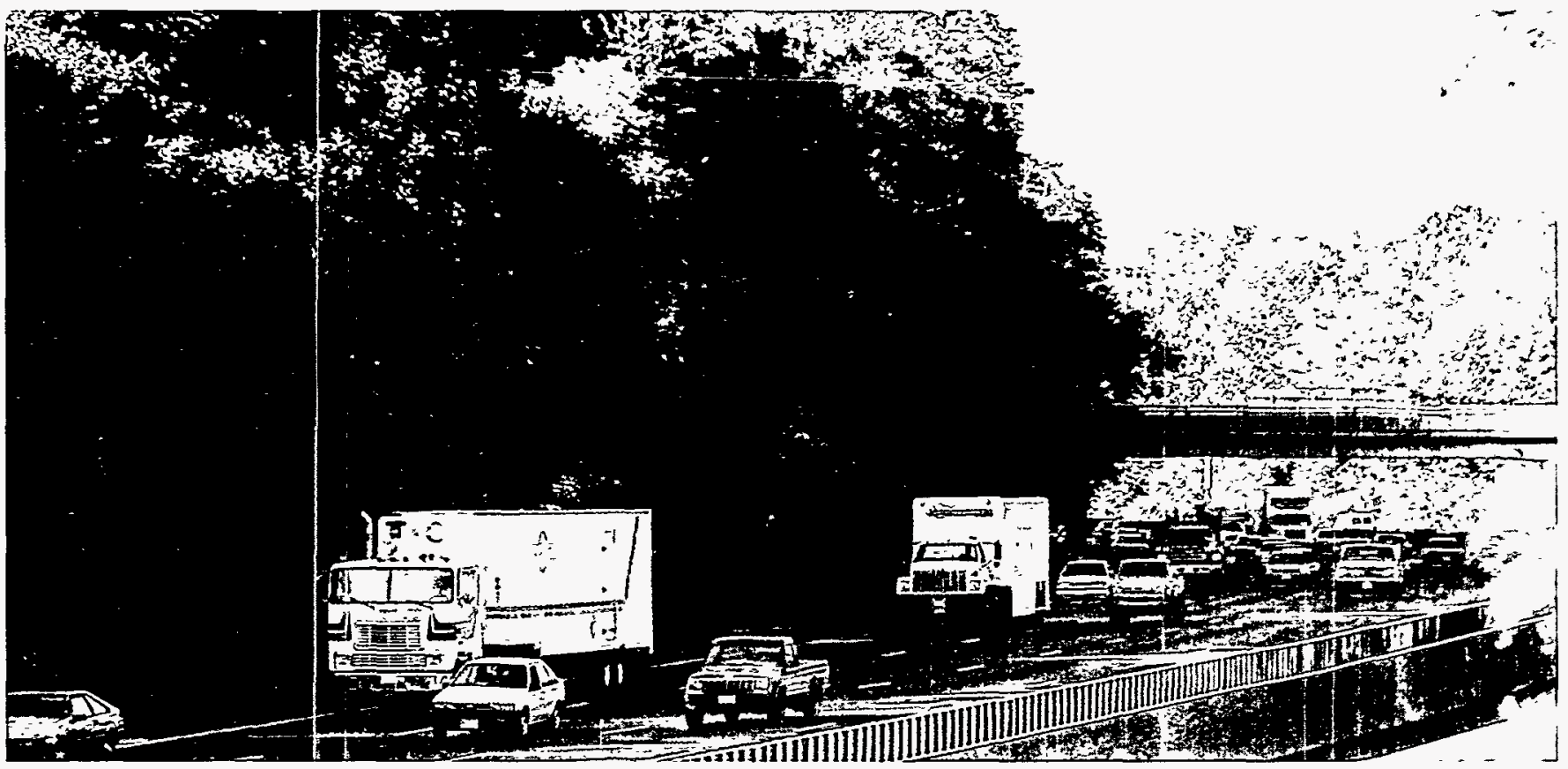

More diesel is used for on-highway use than for any other use. 
Table 14. Adjusted Sales of Residual Fuel Oil by End Use in the United States: 1992-1996 (Thousand Gallons)

\begin{tabular}{|c|c|c|c|c|c|}
\hline \multirow{2}{*}{ End Use } & \multicolumn{5}{|c|}{ Residual Fuel Oil } \\
\hline & 1992 & 1993 & 1994 & 1995 & 1996 \\
\hline 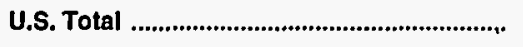 & $16,816,674$ & $16,559,004$ & $15,648,654$ & $13,058,262$ & $13,041,042$ \\
\hline 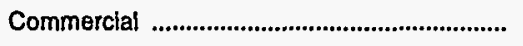 & $1,289,987$ & $1,217,796$ & $1,171,495$ & $1,007,962$ & 944,717 \\
\hline Industria! & $2,332,911$ & $2,823,049$ & $2,579,494$ & $2,160,606$ & $2,127,457$ \\
\hline 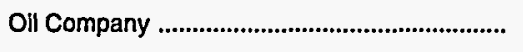 & 297,332 & 316,372 & 267,921 & 231,022 & 170,471 \\
\hline 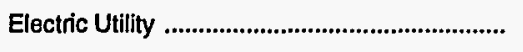 & $5,587,235$ & $5,841,192$ & $5,609,752$ & $3,164,462$ & $3,925,629$ \\
\hline 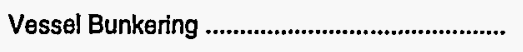 & $7,199,078$ & $6,269,882$ & $5,944,838$ & $6,431,238$ & $5,804,977$ \\
\hline 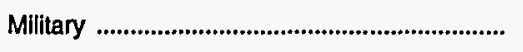 & 100,684 & 86,109 & 68,368 & 55,614 & 57,249 \\
\hline All Other & 9,448 & 4,604 & 6,745 & 7,359 & 10,542 \\
\hline
\end{tabular}

Note: Totals may not equal sum of components due to independent rounding.

Note: Residual fuel oil data in the Adjusted Sales tables (13-24) are adjusted at the national level. See Technical Note 3 for further explanation.

Sources: - Energy Information Administration Form ElA-821, "Annual Fuel Oil and Kerosene Sales Report," for $1992-1996$.

- Electric Utility data are based on data obtained on Form EIA-759, "Monthly Power Plant Report," and Form FERC-423, "Monthly Report of Cost and Quality of Fuels for Electric Plants."

- Energy Information Administration's "Petroleum Supply Annual," Volume 1, 1992-1996, (DOE/EIA-0340(92)/1 - (96)/1).

Table 15. Adjusted Sales of Kerosene by End Use in the United States: 1992-1996 (Thousand Gallons)

\begin{tabular}{|c|c|c|c|c|c|}
\hline \multirow{2}{*}{ End Use } & \multicolumn{5}{|c|}{ Kerosene } \\
\hline & 1992 & 1993 & 1994 & 1995 & 1996 \\
\hline U.S. Total & 636,426 & 760,788 & 750,330 & 828,450 & 948,990 \\
\hline 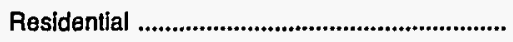 & 481,511 & 560,103 & 480,916 & 550,336 & 657,636 \\
\hline Commercial & 82,398 & 103,996 & 144,142 & 163,716 & 155,792 \\
\hline 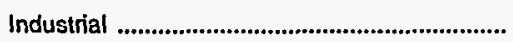 & 46,099 & 58,190 & 86,629 & 86,902 & 102,322 \\
\hline Farm & 20,036 & 27,695 & 27,686 & 19,919 & 26,017 \\
\hline 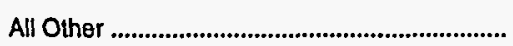 & 6,382 & 10,805 & 10,957 & 7,576 & 7,223 \\
\hline
\end{tabular}

Note: Totals may not equal sum of components due to independent rounding.

Note: Kerosene data in the Adjusted Sales tables (13-24) are adjusted at the national level. See Technical Note 3 for further explanation.

Sources: - Kerosene data are based on data from the Energy Information Administration's "Petroleum Supply Annual," Volume 1, 1992-1996, (DOE/EIA-0340(92)/1 - (96)/1). 
Table 16. Adjusted Sales of Distillate Fuel Oil by End Use, 1995 and 1996 (Thousand Gallons)

\begin{tabular}{|c|c|c|c|c|c|c|}
\hline \multirow{2}{*}{ Destlnation } & \multicolumn{2}{|c|}{ Residential } & \multicolumn{2}{|c|}{ Commercial } & \multicolumn{2}{|c|}{ Industrial } \\
\hline & 1995 & 1996 & 1995 & 1996 & 1995 & 1996 \\
\hline 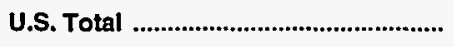 & $6,370,381$ & $6,707,714$ & $3,318,818$ & $3,433,555$ & $2,027,409$ & $2,107,190$ \\
\hline 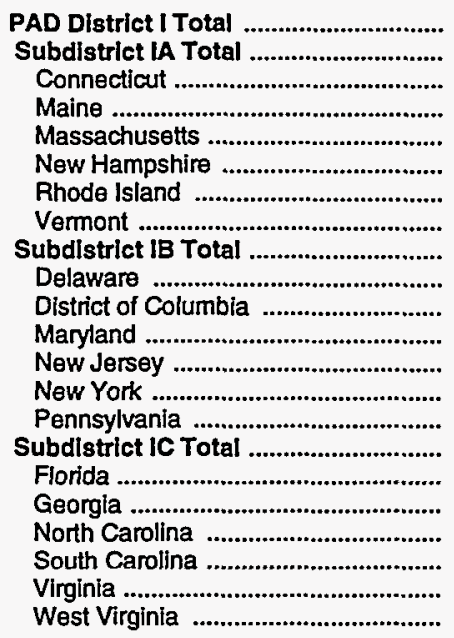 & $\begin{array}{r}5,230,793 \\
2,053,812 \\
509,969 \\
310,466 \\
816,733 \\
181,071 \\
141,080 \\
94,494 \\
2,738,813 \\
45,306 \\
11,575 \\
200,384 \\
489,693 \\
1,165,204 \\
826,651 \\
438,168 \\
9,273 \\
6,677 \\
163,759 \\
28,157 \\
210,110 \\
20,193\end{array}$ & $\begin{array}{r}5,610,264 \\
2,114,262 \\
562,736 \\
321,766 \\
782,640 \\
197,889 \\
148,306 \\
100,925 \\
2,997,204 \\
46,513 \\
12,879 \\
247,702 \\
518,691 \\
1,288,927 \\
882,491 \\
498,798 \\
9,083 \\
6,430 \\
181,453 \\
30,344 \\
245,942 \\
25,547\end{array}$ & $\begin{array}{r}2,231,502 \\
583,812 \\
122,824 \\
93,004 \\
263,716 \\
45,946 \\
30,160 \\
28,162 \\
1,209,806 \\
11,459 \\
33,777 \\
126,085 \\
141,133 \\
639,527 \\
257,826 \\
437,884 \\
119,833 \\
59,140 \\
95,456 \\
40,794 \\
108,137 \\
14,523\end{array}$ & $\begin{array}{r}2,383,023 \\
594,280 \\
126,094 \\
103,298 \\
240,265 \\
56,274 \\
34,445 \\
33,904 \\
1,331,590 \\
16,316 \\
40,964 \\
139,366 \\
210,732 \\
661,993 \\
262,220 \\
457,154 \\
90,379 \\
49,268 \\
120,367 \\
41,076 \\
144,831 \\
11,234\end{array}$ & $\begin{array}{r}699,573 \\
95,439 \\
19,974 \\
25,623 \\
28,613 \\
8,583 \\
7,121 \\
5,525 \\
219,933 \\
6,442 \\
173 \\
24,853 \\
48,129 \\
51,832 \\
88,504 \\
384,201 \\
59,559 \\
39,208 \\
73,053 \\
24,906 \\
69,042 \\
118,433\end{array}$ & $\begin{array}{r}746,115 \\
95,792 \\
22,245 \\
26,922 \\
24,058 \\
6,589 \\
9,291 \\
6,687 \\
232,274 \\
8,190 \\
448 \\
36,982 \\
43,285 \\
48,266 \\
95,102 \\
418,049 \\
46,436 \\
60,485 \\
87,182 \\
27,157 \\
78,180 \\
118,609\end{array}$ \\
\hline 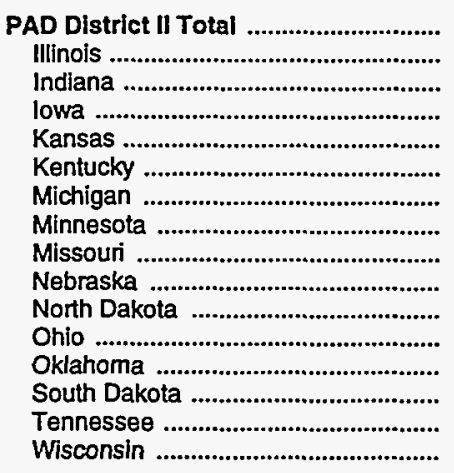 & $\begin{array}{r}923,558 \\
34,576 \\
67,067 \\
35,476 \\
649 \\
32,835 \\
173,335 \\
140,184 \\
19,828 \\
4,000 \\
32,569 \\
181,673 \\
494 \\
22,775 \\
11,820 \\
166,276\end{array}$ & $\begin{array}{r}885,239 \\
31,780 \\
61,655 \\
32,976 \\
740 \\
28,220 \\
164,389 \\
147,025 \\
14,078 \\
4,819 \\
34,842 \\
160,908 \\
992 \\
26,546 \\
11,444 \\
164,824\end{array}$ & $\begin{array}{r}593,639 \\
84,979 \\
50,155 \\
18,859 \\
25,553 \\
50,597 \\
74,420 \\
39,150 \\
54,079 \\
7,344 \\
6,723 \\
77,648 \\
12,267 \\
13,674 \\
33,558 \\
44,635\end{array}$ & $\begin{array}{r}565,136 \\
77,447 \\
41,097 \\
15,175 \\
23,606 \\
50,824 \\
75,236 \\
43,179 \\
55,765 \\
9,816 \\
8,881 \\
56,879 \\
16,312 \\
10,679 \\
38,583 \\
41,657\end{array}$ & $\begin{array}{r}658,340 \\
59,580 \\
67,786 \\
16,315 \\
9,840 \\
195,758 \\
41,584 \\
44,842 \\
27,005 \\
4,055 \\
10,744 \\
83,688 \\
5,939 \\
8,461 \\
32,698 \\
50,045\end{array}$ & $\begin{array}{r}649,804 \\
52,819 \\
70,004 \\
20,597 \\
10,601 \\
183,408 \\
43,278 \\
38,907 \\
33,797 \\
4,115 \\
6,683 \\
73,064 \\
8,899 \\
9,654 \\
39,956 \\
54,023\end{array}$ \\
\hline 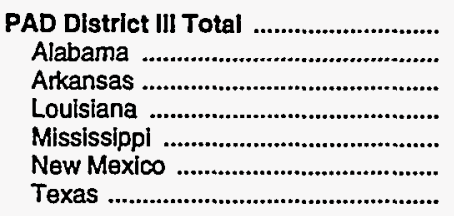 & $\begin{array}{r}817 \\
363 \\
71 \\
49 \\
17 \\
100 \\
217\end{array}$ & $\begin{array}{r}556 \\
371 \\
33 \\
24 \\
23 \\
103 \\
3\end{array}$ & $\begin{array}{r}154,060 \\
22,385 \\
10,466 \\
8,947 \\
11,051 \\
8,415 \\
92,796\end{array}$ & $\begin{array}{r}156,172 \\
20,509 \\
10,724 \\
4,958 \\
14,654 \\
6,483 \\
98,844\end{array}$ & $\begin{array}{r}228,026 \\
48,915 \\
11,870 \\
14,077 \\
19,523 \\
19,973 \\
113,668\end{array}$ & $\begin{array}{r}246,818 \\
52,872 \\
9,593 \\
20,812 \\
22,060 \\
25,601 \\
115,881\end{array}$ \\
\hline 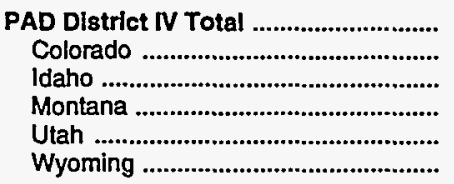 & $\begin{array}{r}39,542 \\
1,699 \\
21,427 \\
10,595 \\
3,515 \\
2,307\end{array}$ & $\begin{array}{r}48,833 \\
2,528 \\
22,112 \\
18,422 \\
4,217 \\
1,554\end{array}$ & $\begin{array}{r}89,802 \\
34,217 \\
19,082 \\
4,963 \\
18,615 \\
12,924\end{array}$ & $\begin{array}{r}116,291 \\
41,460 \\
25,733 \\
12,947 \\
21,193 \\
14,958\end{array}$ & $\begin{array}{r}151,288 \\
19,123 \\
31,016 \\
29,128 \\
20,946 \\
51,074\end{array}$ & $\begin{array}{r}175,221 \\
19,100 \\
29,990 \\
24,327 \\
26,713 \\
75,092\end{array}$ \\
\hline 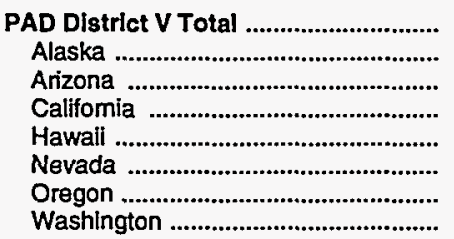 & $\begin{array}{r}175,671 \\
62,800 \\
179 \\
5,433 \\
56 \\
5,459 \\
39,591 \\
62,151\end{array}$ & $\begin{array}{r}162,821 \\
55,141 \\
276 \\
4,227 \\
7 \\
5,674 \\
34,491 \\
63,006\end{array}$ & $\begin{array}{r}249,814 \\
32,097 \\
10,991 \\
98,146 \\
10,651 \\
25,796 \\
32,928 \\
39,205\end{array}$ & $\begin{array}{r}212,933 \\
33,786 \\
16,925 \\
73,223 \\
6,400 \\
28,243 \\
26,061 \\
28,295\end{array}$ & $\begin{array}{r}290,182 \\
34,756 \\
63,157 \\
55,527 \\
2,272 \\
93,907 \\
19,362 \\
21,200\end{array}$ & $\begin{array}{r}289,232 \\
40,800 \\
72,582 \\
37,738 \\
2,523 \\
96,650 \\
23,376 \\
15,562\end{array}$ \\
\hline
\end{tabular}

See footnotes at end of table. 
Table 16. Adjusted Sales of Distillate Fuel Oil by End Use, 1995 and 1996 (Continued) (Thousand Gallons)

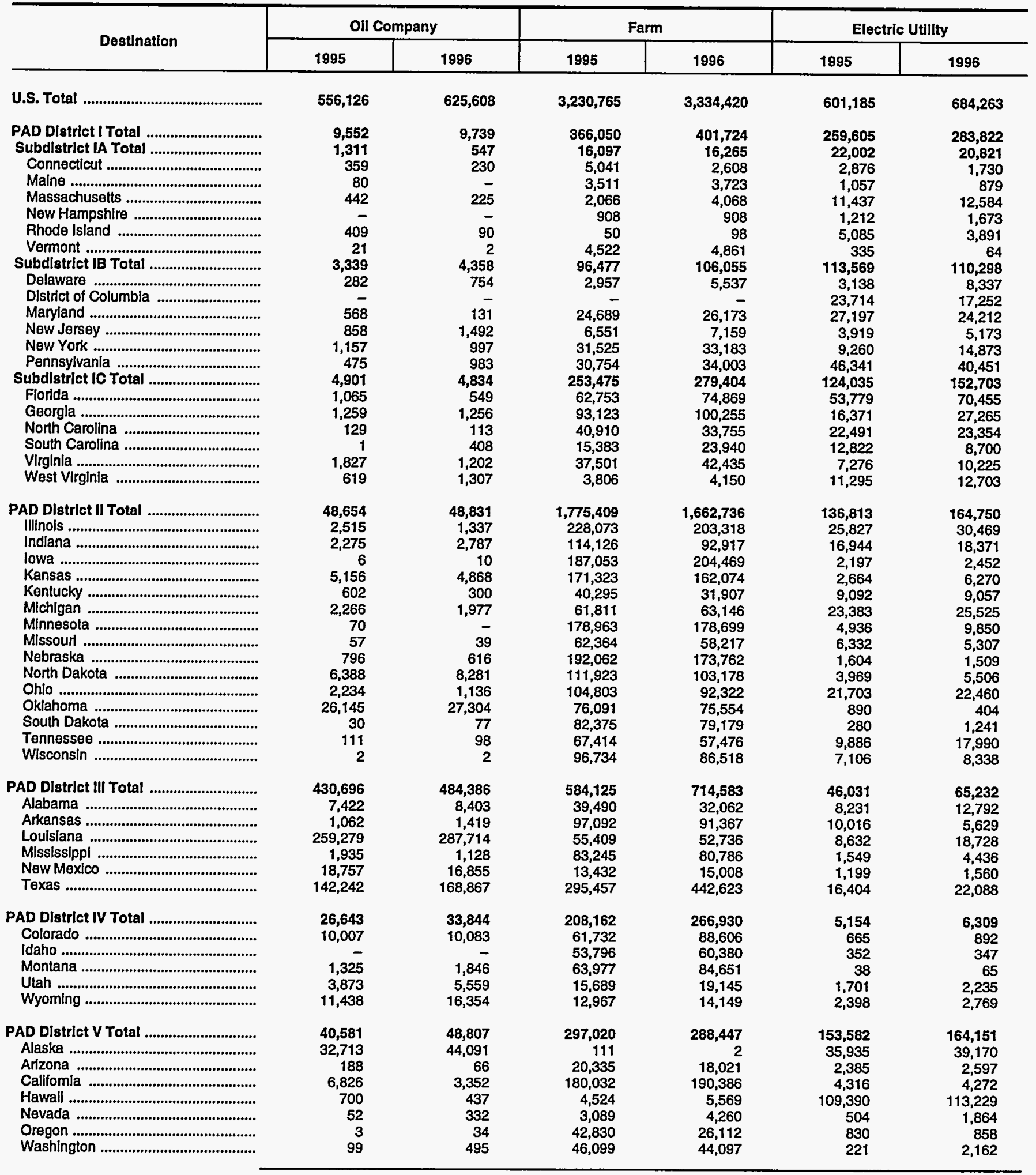

See footnotes at end of table. 
Table 16. Adjusted Sales of Distillate Fuel Oil by End Use, 1995 and 1996 (Continued)

(Thousand Gallons)

\begin{tabular}{|c|c|c|c|c|c|c|}
\hline \multirow{2}{*}{ Destination } & \multicolumn{2}{|c|}{ Rallroad } & \multicolumn{2}{|c|}{ Vessel Bunkering } & \multicolumn{2}{|c|}{ On-Highway } \\
\hline & 1995 & 1996 & 1995 & 1996 & 1995 & 1996 \\
\hline 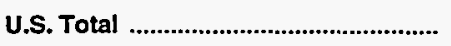 & $3,191,023$ & $3,266,861$ & $1,978,105$ & $2,177,608$ & $25,576,433$ & $26,958,668$ \\
\hline 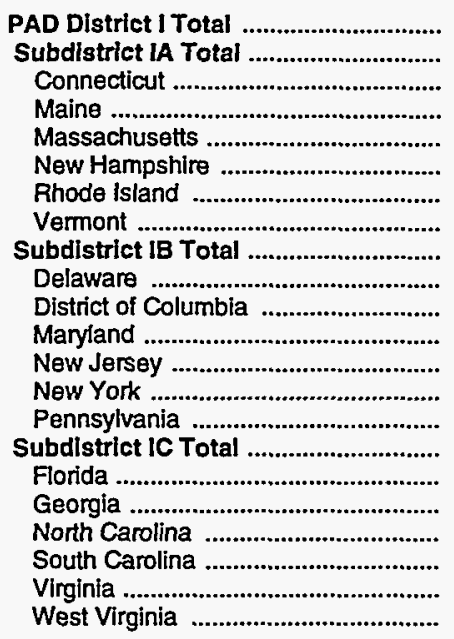 & $\begin{array}{r}517,700 \\
27,333 \\
6,256 \\
6,261 \\
13,982 \\
281 \\
17 \\
536 \\
180,176 \\
427 \\
5,301 \\
26,033 \\
72,148 \\
10,672 \\
65,595 \\
310,191 \\
86,824 \\
65,935 \\
69,573 \\
6,624 \\
67,787 \\
13,449\end{array}$ & $\begin{array}{r}568,371 \\
24,862 \\
6,215 \\
5,738 \\
12,132 \\
149 \\
96 \\
532 \\
143,614 \\
415 \\
6,161 \\
28,371 \\
63,791 \\
7,041 \\
37,836 \\
399,895 \\
103,973 \\
76,841 \\
72,825 \\
11,176 \\
128,748 \\
6,332\end{array}$ & $\begin{array}{r}499,385 \\
74,504 \\
3,095 \\
18,095 \\
44,055 \\
306 \\
8,529 \\
424 \\
147,079 \\
1,247 \\
12 \\
19,219 \\
100,016 \\
10,936 \\
15,648 \\
277,802 \\
154,038 \\
14,147 \\
16,722 \\
3,759 \\
28,866 \\
60,271\end{array}$ & $\begin{array}{r}513,469 \\
71,917 \\
2,472 \\
12,479 \\
46,282 \\
737 \\
9,481 \\
466 \\
138,053 \\
6,381 \\
2 \\
20,245 \\
81,076 \\
13,882 \\
16,467 \\
303,498 \\
166,043 \\
10,399 \\
19,332 \\
10,878 \\
26,093 \\
70,752\end{array}$ & $\begin{array}{r}8,099,324 \\
837,751 \\
199,252 \\
129,755 \\
315,292 \\
63,113 \\
44,142 \\
86,197 \\
3,007,852 \\
62,912 \\
21,205 \\
323,559 \\
488,531 \\
915,846 \\
1,195,799 \\
4,253,721 \\
1,003,655 \\
1,107,982 \\
774,747 \\
460,194 \\
688,044 \\
219,099\end{array}$ & $\begin{array}{r}8,372,128 \\
858,402 \\
E_{208,249} \\
135,339 \\
313,984 \\
60,949 \\
44,378 \\
95,503 \\
3,063,474 \\
59,290 \\
20,949 \\
355,412 \\
532,936 \\
924,782 \\
1,170,105 \\
4,450,252 \\
E_{962,504} \\
1,345,755 \\
794,837 \\
458,312 \\
757,666 \\
131,178\end{array}$ \\
\hline 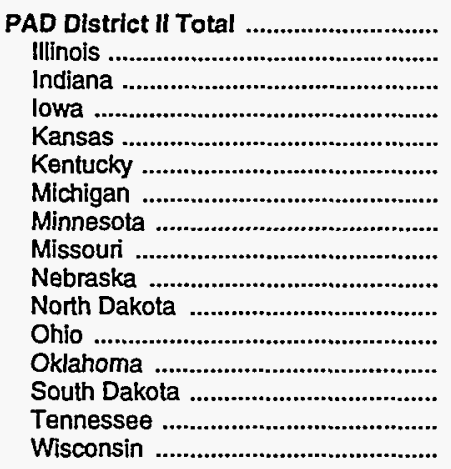 & $\begin{array}{r}1,468,768 \\
182,919 \\
145,163 \\
60,472 \\
221,920 \\
116,553 \\
62,233 \\
114,729 \\
21,326 \\
111,954 \\
46,120 \\
130,977 \\
100,183 \\
4,797 \\
95,440 \\
53,984\end{array}$ & $\begin{array}{r}1,412,083 \\
200,798 \\
127,691 \\
66,190 \\
121,763 \\
104,310 \\
43,626 \\
71,590 \\
22,782 \\
165,708 \\
46,467 \\
135,184 \\
152,129 \\
1,957 \\
96,740 \\
55,149\end{array}$ & $\begin{array}{r}427,962 \\
50,558 \\
15,812 \\
6,542 \\
- \\
150,574 \\
3,778 \\
5,311 \\
88,875 \\
- \\
- \\
7,771 \\
527 \\
- \\
94,976 \\
3,239\end{array}$ & $\begin{array}{r}477,296 \\
46,912 \\
16,934 \\
7,011 \\
- \\
146,330 \\
4,753 \\
4,835 \\
163,421 \\
49 \\
- \\
8,447 \\
147 \\
- \\
76,352 \\
2,103\end{array}$ & $\begin{array}{r}8,572,145 \\
E_{845,546} \\
976,069 \\
409,958 \\
343,175 \\
581,148 \\
735,953 \\
453,926 \\
737,909 \\
312,127 \\
131,234 \\
1,100,569 \\
496,860 \\
135,905 \\
728,024 \\
583,742\end{array}$ & $\begin{array}{r}9,125,173 \\
884,696 \\
1,036,200 \\
458,660 \\
352,872 \\
587,549 \\
772,262 \\
482,131 \\
767,383 \\
336,764 \\
135,043 \\
1,275,402 \\
541,337 \\
141,728 \\
754,982 \\
598,164\end{array}$ \\
\hline 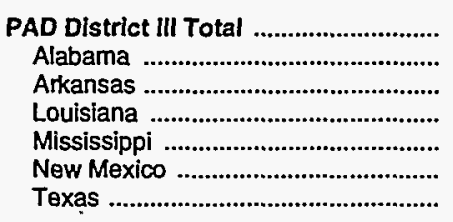 & $\begin{array}{r}524,890 \\
41,458 \\
49,196 \\
17,826 \\
22,317 \\
14,375 \\
379,717\end{array}$ & $\begin{array}{r}554,336 \\
44,929 \\
57,333 \\
23,771 \\
21,128 \\
19,045 \\
388,130\end{array}$ & $\begin{array}{r}748,086 \\
49,575 \\
2,446 \\
444,237 \\
19,978 \\
- \\
231,850\end{array}$ & $\begin{array}{r}946,627 \\
55,758 \\
2,830 \\
608,963 \\
23,075 \\
- \\
256,001\end{array}$ & $\begin{array}{r}4,089,230 \\
691,273 \\
488,499 \\
481,346 \\
374,214 \\
107,055 \\
1,946,843\end{array}$ & $\begin{array}{r}4,522,044 \\
646,567 \\
495,380 \\
508,414 \\
400,100 \\
314,590 \\
2,156,993\end{array}$ \\
\hline 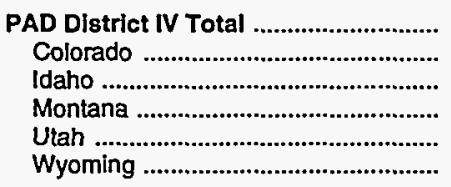 & $\begin{array}{r}377,048 \\
115,691 \\
32,537 \\
87,387 \\
34,874 \\
106,559\end{array}$ & $\begin{array}{r}450,930 \\
130,661 \\
37,901 \\
93,160 \\
53,266 \\
135,942\end{array}$ & $\begin{array}{r}60 \\
- \\
37 \\
4 \\
20 \\
-\end{array}$ & $\begin{array}{r}339 \\
- \\
314 \\
5 \\
20 \\
-\end{array}$ & $\begin{array}{r}1,117,000 \\
276,585 \\
167,819 \\
158,451 \\
258,135 \\
256,010\end{array}$ & $\begin{array}{r}1,094,512 \\
273,207 \\
187,828 \\
140,625 \\
255,192 \\
237,660\end{array}$ \\
\hline 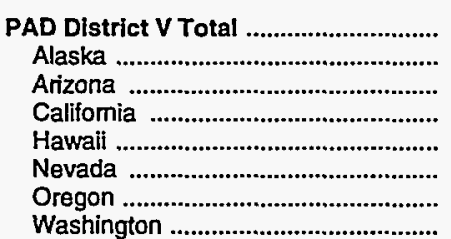 & $\begin{array}{r}302,617 \\
3,118 \\
3,241 \\
166,057 \\
- \\
11,132 \\
65,064 \\
54,005\end{array}$ & $\begin{array}{r}281,141 \\
2,959 \\
2,465 \\
166,165 \\
- \\
5,452 \\
54,651 \\
49,448\end{array}$ & $\begin{array}{r}302,612 \\
103,629 \\
14 \\
73,101 \\
58,171 \\
- \\
15,467 \\
52,230\end{array}$ & $\begin{array}{r}239,877 \\
94,617 \\
11 \\
65,966 \\
15,613 \\
- \\
15,551 \\
48,119\end{array}$ & $\begin{array}{r}3,698,734 \\
109,740 \\
483,186 \\
2,089,687 \\
27,080 \\
E_{172,332} \\
E_{353,384} \\
463,325\end{array}$ & $\begin{array}{r}3,844,811 \\
34,918 \\
543,643 \\
2,115,704 \\
40,447 \\
E_{244,609} \\
386,239 \\
479,251\end{array}$ \\
\hline
\end{tabular}

See footnotes at end of table. 
Table 16. Adjusted Sales of Distillate Fuel Oil by End Use, 1995 and 1996 (Continued) (Thousand Gallons)

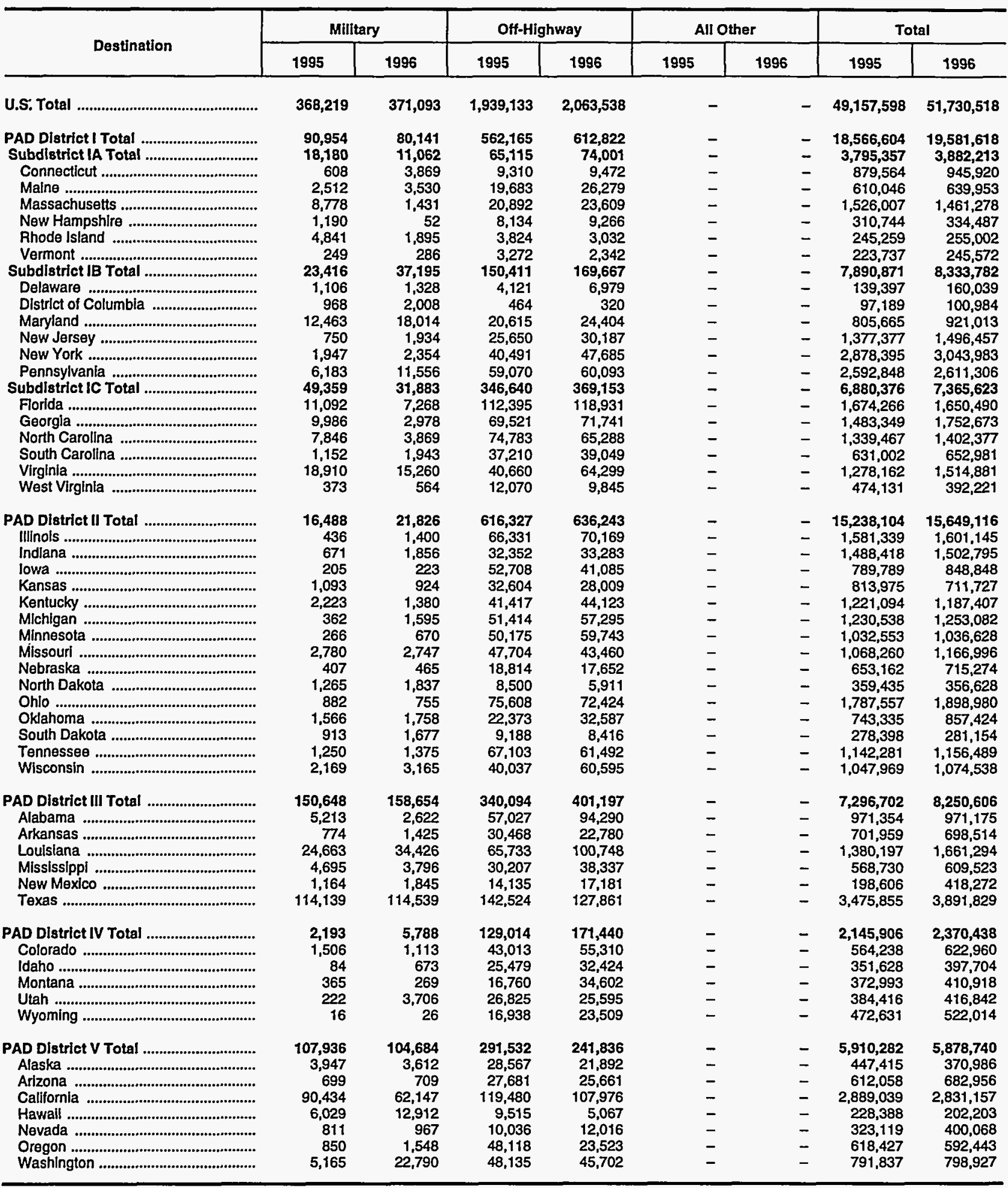

E Estimated data. Florida's 1996 estimate is based on seven months of data. Nevada's 1996 estimate is based on eight months of data. Illinois' and Nevada's 1995 data are based on eleven months of data. Oregon's 1995 and Connecticut's 1996 data are a Federal Highway Administration estimate.

Dashes $(-)=$ No data reported.

Note: Totals may not equal sum of components due to independent rounding.

Sources: - Energy Information Administration Form ElA-821, "Annual Fuel Oil and Kerosene Sales Report," for 1995 and 1996.

- Electric Utility data are based on data obtained on Form EIA-759, "Monthly Power Plant Report," and Form FEAC-423, "Monthly Report of Cost and Quality of Fuels for Electric Plants."

- On-Highway Dlesel data are derived from Federal Highway Administation statistics of highway special fuels use. 
Table 17. Adjusted Sales of Residual Fuel Oil by End Use, 1995 and 1996 (Thousand Gallons)

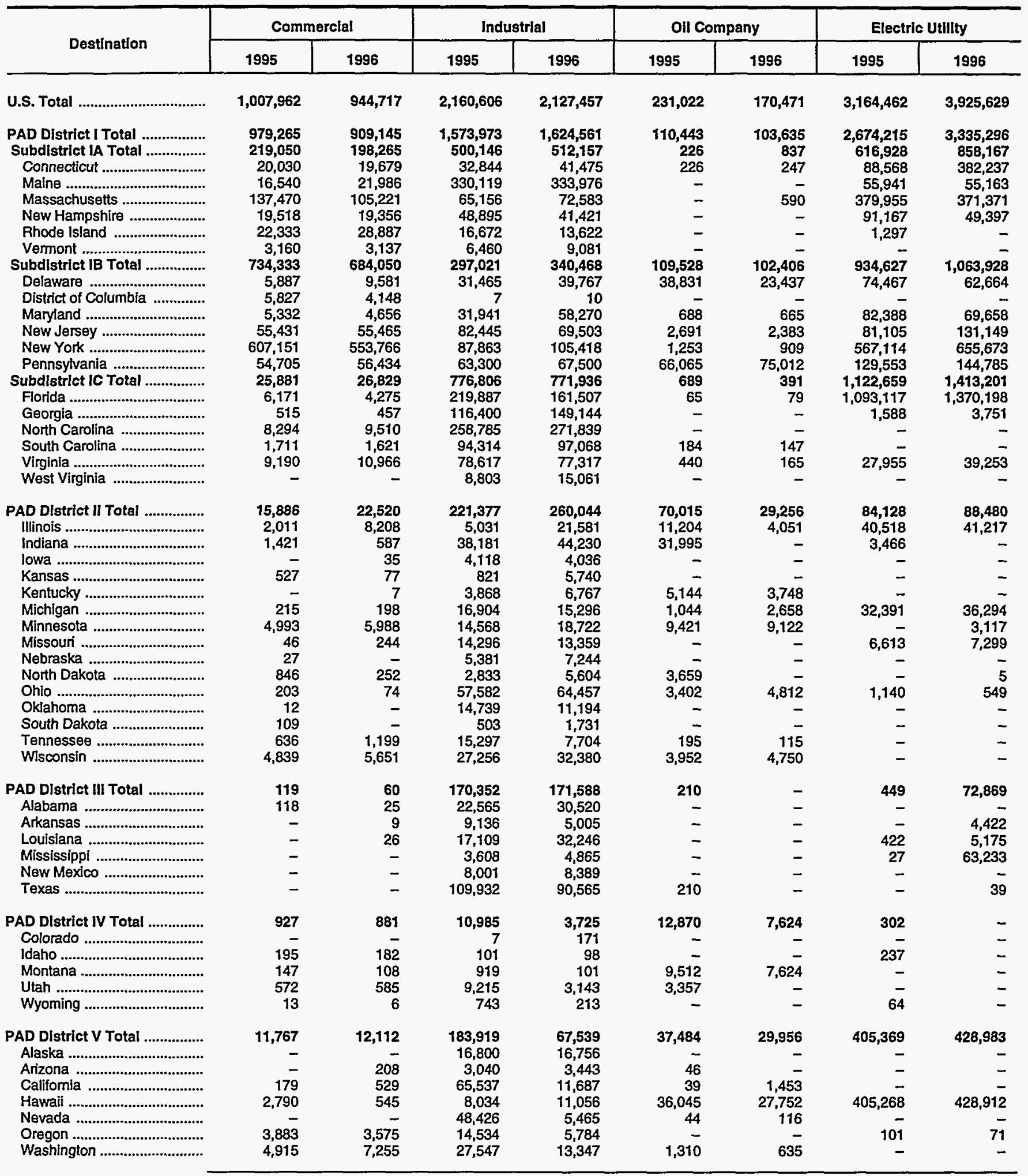

See footnotes at end of table. 
Table 17. Adjusted Sales of Residual Fuel Oil by End Use, 1995 and 1996 (Continued) (Thousand Gallons)

\begin{tabular}{|c|c|c|c|c|c|c|c|c|}
\hline \multirow{2}{*}{ Destlnation } & \multicolumn{2}{|c|}{ Vessel Bunkering } & \multicolumn{2}{|c|}{ Milltary } & \multicolumn{2}{|c|}{ All Other } & \multicolumn{2}{|c|}{ Total } \\
\hline & 1995 & 1996 & 1995 & 1996 & 1995 & 1996 & 1995 & 1996 \\
\hline 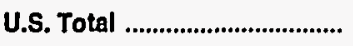 & $6,431,238$ & $5,804,977$ & 55,614 & 57,249 & 7,359 & 10,542 & $13,058,262$ & $13,041,042$ \\
\hline PAD Dlstrict I Total ................. & $1,299,435$ & $1,360,439$ & 43,676 & 40,120 & 5,002 & 7,792 & $6,686,008$ & $7,380,987$ \\
\hline 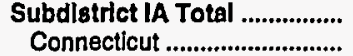 & $\begin{array}{r}10,652 \\
-\end{array}$ & $\begin{array}{r}86,070 \\
177\end{array}$ & $\begin{array}{r}7,993 \\
514\end{array}$ & $\begin{array}{r}11,212 \\
1,376\end{array}$ & $\begin{array}{r}1,244 \\
741\end{array}$ & $\begin{array}{r}354 \\
-\end{array}$ & $\begin{array}{r}1,356,240 \\
142,923\end{array}$ & $\begin{array}{r}1,667,063 \\
445,191\end{array}$ \\
\hline 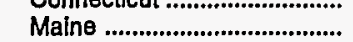 & $4,11 \overline{7}$ & 2,055 & 5,013 & 6,676 & 358 & $35 \overline{4}$ & $\begin{array}{l}142,923 \\
412,089\end{array}$ & $\begin{array}{l}445,191 \\
420,210\end{array}$ \\
\hline Massachusetts ....................... & 6,490 & 83,533 & 2,432 & 3,160 & 144 & - & 591,648 & 636,458 \\
\hline New Hampshire ..................... & - & 212 & - & - & - & - & 159,580 & 110,385 \\
\hline Rhode Island ........................... & 45 & 92 & 34 & - & - & - & 40,381 & 42,601 \\
\hline 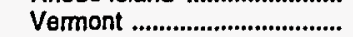 & - & - & - & - & - & - & 9,619 & 12,218 \\
\hline Subdlstrict IB Total ................ & 744,913 & 788,155 & 20,898 & 14,018 & 6 & - & $2,841,327$ & $2,993,025$ \\
\hline 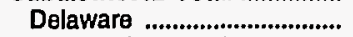 & 44,207 & 85,645 & 1,933 & 806 & 6 & - & 196,796 & 221,901 \\
\hline District of Columbla ................ & - & - & - & - & - & - & 5,833 & 4,158 \\
\hline 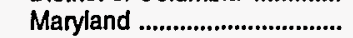 & 31,241 & 25,976 & 10,466 & 6,731 & - & - & 162,056 & 165,955 \\
\hline New Jersey ............................. & 357,584 & 255,860 & 2,941 & 4,289 & - & - & 582,198 & 518,649 \\
\hline New York ............................... & 100,276 & 277,974 & 3,545 & 890 & - & - & $1,367,202$ & $1,594,629$ \\
\hline Pennsylvania ......................... & 211,605 & 142,699 & 2,013 & 1,302 & - & - & 527,241 & 487,732 \\
\hline Subdlatrlct IC Total ................... & 543,869 & 486,214 & 14,785 & 14,890 & 3,752 & 7,438 & $2,488,441$ & $2,720,900$ \\
\hline 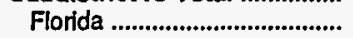 & 375,361 & 350,235 & 2,468 & 1,581 & 3,118 & 7,385 & $1,700,189$ & $1,895,260$ \\
\hline 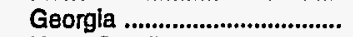 & 61,967 & 53,561 & - & - & - & - & 180,470 & 206,913 \\
\hline North Carolina ....................... & 6,511 & 7,572 & 6,880 & 6,635 & 68 & 53 & 280,538 & 295,610 \\
\hline South Carolina ...................... & 17,769 & 25,732 & 1,581 & 2,920 & 39 & - & 115,598 & 127,487 \\
\hline Virginia ...................................... & 82,261 & 48,940 & 3,856 & 3,754 & 525 & - & 202,843 & 180,395 \\
\hline West Virginia ......................... & - & 174 & - & - & - & - & 8,803 & 15,235 \\
\hline PAD Distrlct \| Total ................ & 20,505 & 24,428 & 338 & 657 & 185 & - & 412,434 & 425,385 \\
\hline 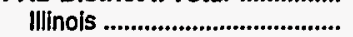 & 1,248 & 1,286 & 338 & 21 & 38 & - & 60,389 & 76,363 \\
\hline 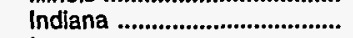 & 10,517 & 12,674 & - & - & - & - & 85,580 & 57,491 \\
\hline lowa & - & - & - & - & 8 & - & 4,126 & 4,070 \\
\hline 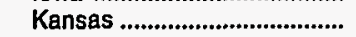 & - & - & - & - & - & - & 1,349 & 5,817 \\
\hline Kentucky .................................. & - & - & - & - & - & - & 9,012 & 10,522 \\
\hline Michigan .............................. & 4,209 & 5,196 & - & 122 & 43 & - & 54,807 & 59,763 \\
\hline 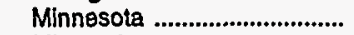 & - & - & - & - & - & - & 28,982 & 36,948 \\
\hline Missouri ................................... & 945 & 247 & - & 514 & - & - & 21,900 & 21,663 \\
\hline Nebraska ............................... & - & - & - & - & 3 & - & 5,411 & 7,244 \\
\hline 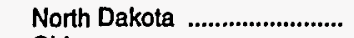 & - & - & - & - & - & - & 7,338 & 5,861 \\
\hline 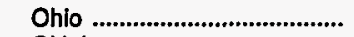 & 2,519 & 3,570 & - & - & - & - & 64,847 & 73,462 \\
\hline Oklahoma ............................... & - & - & - & - & - & - & 14,751 & 11,194 \\
\hline South Dakota .......................... & - & - & - & - & - & - & 611 & 1,731 \\
\hline Tennesseg ............................. & 84 & 90 & - & - & - & - & 16,211 & 9,109 \\
\hline Wisconsin .............................. & 981 & 1,365 & - & - & 92 & - & 37,119 & 44,146 \\
\hline PAD District lil Total ............... & $2,139,498$ & $2,050,735$ & 1,958 & 4,767 & - & 31 & $2,312,585$ & $2,300,050$ \\
\hline 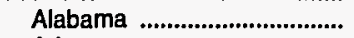 & 116,608 & 105,997 & - & - & - & - & 139,291 & 136,541 \\
\hline 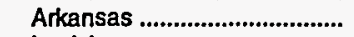 & - & - & - & - & - & - & 9,136 & 9,436 \\
\hline Loulsiana ............................... & $1,013,170$ & $1,101,644$ & 1,958 & 1,881 & - & - & $1,032,660$ & $1,140,972$ \\
\hline 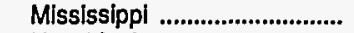 & 112,835 & 72,515 & - & - & - & - & 116,470 & 140,613 \\
\hline New Mexico ........................... & - & - & - & - & - & 31 & 8,001 & 8,420 \\
\hline 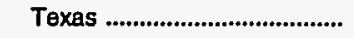 & 896,885 & 770,579 & - & 2,886 & - & - & $1,007,027$ & 864,069 \\
\hline PAD Dlstrlct IV Total .............. & - & - & - & 8 & 228 & 71 & 25,312 & 12,309 \\
\hline 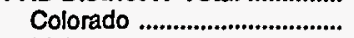 & - & - & - & 8 & 4 & 4 & 11 & 182 \\
\hline 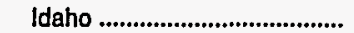 & - & - & - & - & 31 & 5 & 564 & 285 \\
\hline 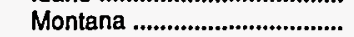 & - & - & - & - & - & - & 10,578 & 7,833 \\
\hline 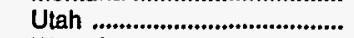 & - & - & - & - & 44 & 24 & 13,188 & 3,752 \\
\hline Wyoming .................................... & - & - & - & - & 150 & 38 & 970 & 257 \\
\hline PAD Dlstrlct V Total ................ & $2,971,800$ & $2,369,375$ & 9,641 & 11,697 & 1,944 & 2,649 & $3,621,924$ & $2,922,311$ \\
\hline 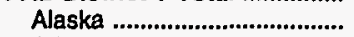 & 5,106 & 152 & - & - & - & - & 21,907 & 16,908 \\
\hline 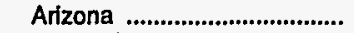 & - & - & - & - & - & - & 3,086 & 3,651 \\
\hline 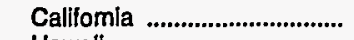 & $1,964,209$ & $1,680,188$ & 8,530 & 7,509 & 114 & 28 & $2,038,608$ & $1,701,395$ \\
\hline 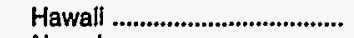 & 119,788 & 26,931 & 131 & 3,460 & 1,806 & 2,621 & 573,863 & 501,276 \\
\hline 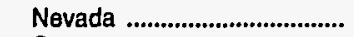 & - & - & - & - & - & - & 48,470 & 5,582 \\
\hline 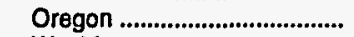 & 141,344 & 131,310 & 980 & - & 24 & - & 160,866 & 140,740 \\
\hline Washington ............................ & 741,353 & 530,794 & - & 729 & - & - & 775,124 & 552,760 \\
\hline
\end{tabular}

Dashes $(-)=$ No data reported.

Note: Totals may not equal sum of components due to independent rounding.

Sources: - Energy Intormation Administration Form EIA-821. "Annual Fuel Oil and Kerosene Sales Report," for 1995 and 1996

- Electric Utility data are based on data obtained on Form EIA-759, "Monthly Power Plant Report," and Form FERC-423, "Monthly Report of Cost and Quality of Fuels for Electric Plants."

- Energy Information Administration's "Petroleum Supply Annual," Volume 1, 1995 and 1996, (DOE/E1A-0340(95)/1 - (96)/1). 
Table 18. Adjusted Sales of Kerosene by End Use, 1995 and 1996 (Thousand Gallons)

\begin{tabular}{|c|c|c|c|c|c|c|}
\hline \multirow{2}{*}{ Destination } & \multicolumn{2}{|c|}{ Residential } & \multicolumn{2}{|c|}{ Commercial } & \multicolumn{2}{|c|}{ Industrial } \\
\hline & 1995 & 1996 & 1995 & 1996 & 1995 & 1996 \\
\hline U.S. Total & 550,336 & 657,636 & 163,716 & 155,792 & 86,902 & 102,322 \\
\hline 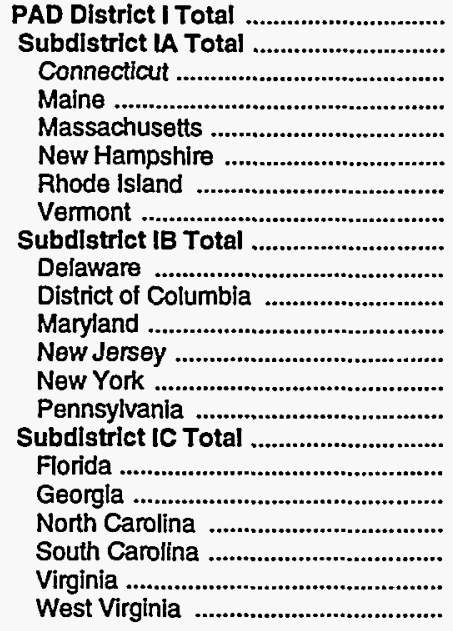 & $\begin{array}{r}440,639 \\
78,901 \\
5,127 \\
45,725 \\
5,463 \\
13,901 \\
1,123 \\
7,563 \\
176,460 \\
5,037 \\
265 \\
22,450 \\
9,926 \\
52,099 \\
86,682 \\
185,278 \\
8,846 \\
5,278 \\
88,104 \\
19,751 \\
51,244 \\
12,055\end{array}$ & $\begin{array}{r}530,407 \\
95,280 \\
5,217 \\
57,548 \\
6,219 \\
16,486 \\
1,268 \\
8,542 \\
206,836 \\
7,574 \\
259 \\
24,925 \\
11,912 \\
60,898 \\
101,268 \\
228,291 \\
11,067 \\
6,047 \\
106,932 \\
23,570 \\
64,847 \\
15,829\end{array}$ & $\begin{array}{r}132,354 \\
16,254 \\
1,144 \\
6,783 \\
4,615 \\
1,834 \\
1,278 \\
600 \\
90,240 \\
99 \\
5,402 \\
8,807 \\
23,761 \\
30,006 \\
22,166 \\
25,859 \\
4,002 \\
1,477 \\
6,178 \\
1,094 \\
11,566 \\
1,542\end{array}$ & $\begin{array}{r}116,949 \\
13,593 \\
3,012 \\
6,222 \\
1,965 \\
1,746 \\
82 \\
566 \\
75,937 \\
260 \\
4,251 \\
6,324 \\
10,216 \\
31,554 \\
23,333 \\
27,418 \\
4,445 \\
1,311 \\
7,496 \\
977 \\
11,651 \\
1,538\end{array}$ & $\begin{array}{r}59,357 \\
7,148 \\
3,904 \\
933 \\
1,365 \\
603 \\
284 \\
60 \\
40,764 \\
10 \\
- \\
1,520 \\
17,257 \\
16,640 \\
5,337 \\
11,444 \\
210 \\
1,053 \\
3,429 \\
2,849 \\
1,838 \\
2,065\end{array}$ & $\begin{array}{r}65,977 \\
2,962 \\
1,062 \\
453 \\
474 \\
435 \\
119 \\
420 \\
47,702 \\
2,017 \\
- \\
1,305 \\
13,030 \\
27,225 \\
4,125 \\
15,312 \\
1,230 \\
1,379 \\
5,399 \\
3,417 \\
1,485 \\
2,401\end{array}$ \\
\hline 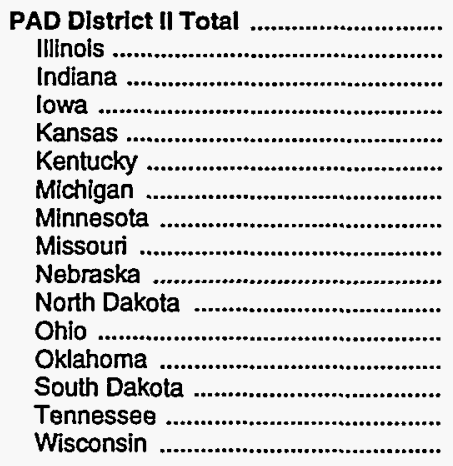 & $\begin{array}{r}93,893 \\
3,521 \\
9,016 \\
1,032 \\
533 \\
17,428 \\
9,772 \\
2,097 \\
1,357 \\
157 \\
154 \\
31,432 \\
156 \\
156 \\
15,644 \\
1,439\end{array}$ & $\begin{array}{r}107,822 \\
4,020 \\
12,096 \\
1,247 \\
808 \\
18,410 \\
9,646 \\
2,558 \\
2,368 \\
159 \\
224 \\
34,377 \\
843 \\
216 \\
19,139 \\
1,711\end{array}$ & $\begin{array}{r}25,255 \\
3,375 \\
2,947 \\
106 \\
236 \\
4,925 \\
4,285 \\
979 \\
432 \\
168 \\
54 \\
3,727 \\
216 \\
30 \\
3,362 \\
412\end{array}$ & $\begin{array}{r}30,506 \\
2,826 \\
2,887 \\
184 \\
208 \\
4,649 \\
6,278 \\
1,123 \\
1,138 \\
167 \\
74 \\
6,507 \\
210 \\
25 \\
3,722 \\
510\end{array}$ & $\begin{array}{r}15,373 \\
3,991 \\
1,221 \\
205 \\
90 \\
2,813 \\
894 \\
106 \\
111 \\
27 \\
2 \\
4,355 \\
104 \\
3 \\
1,110 \\
341\end{array}$ & $\begin{array}{r}19,453 \\
4,917 \\
1,903 \\
118 \\
115 \\
3,092 \\
937 \\
97 \\
1,034 \\
44 \\
3 \\
5,204 \\
181 \\
8 \\
1,357 \\
444\end{array}$ \\
\hline 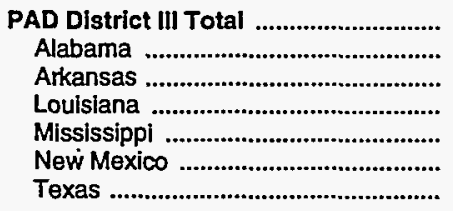 & $\begin{array}{r}5,758 \\
2,789 \\
584 \\
380 \\
847 \\
235 \\
923\end{array}$ & $\begin{array}{r}6,706 \\
2,675 \\
493 \\
707 \\
941 \\
274 \\
1,616\end{array}$ & $\begin{array}{r}3,241 \\
402 \\
190 \\
250 \\
312 \\
147 \\
1,940\end{array}$ & $\begin{array}{r}2,768 \\
392 \\
222 \\
313 \\
233 \\
31 \\
1,577\end{array}$ & $\begin{array}{r}8,889 \\
1,739 \\
422 \\
796 \\
724 \\
220 \\
4,987\end{array}$ & $\begin{array}{r}10,679 \\
1,988 \\
299 \\
971 \\
796 \\
331 \\
6,293\end{array}$ \\
\hline 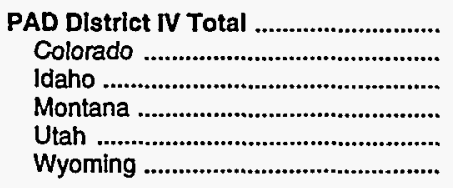 & $\begin{array}{r}1,632 \\
836 \\
627 \\
21 \\
121 \\
27\end{array}$ & $\begin{array}{r}1,626 \\
868 \\
526 \\
28 \\
176 \\
29\end{array}$ & $\begin{array}{r}441 \\
190 \\
128 \\
11 \\
45 \\
66\end{array}$ & $\begin{array}{r}585 \\
245 \\
153 \\
20 \\
119 \\
49\end{array}$ & $\begin{array}{r}1,022 \\
55 \\
4 \\
3 \\
50 \\
909\end{array}$ & $\begin{array}{r}1,158 \\
62 \\
7 \\
3 \\
73 \\
1,013\end{array}$ \\
\hline 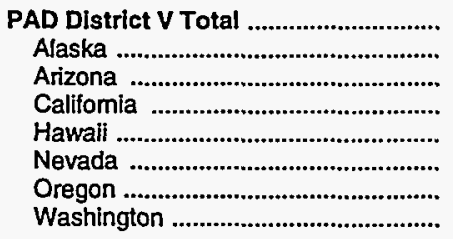 & $\begin{array}{r}8,415 \\
13 \\
85 \\
3,397 \\
1 \\
240 \\
1,072 \\
3,607\end{array}$ & $\begin{array}{r}11,075 \\
12 \\
118 \\
4,343 \\
1 \\
263 \\
1,700 \\
4,640\end{array}$ & $\begin{array}{r}2,426 \\
20 \\
49 \\
1,122 \\
5 \\
53 \\
594 \\
582\end{array}$ & $\begin{array}{r}4,984 \\
6 \\
102 \\
2,889 \\
2 \\
64 \\
1,580 \\
342\end{array}$ & $\begin{array}{r}2,263 \\
5 \\
18 \\
1,782 \\
14 \\
53 \\
103 \\
287\end{array}$ & $\begin{array}{r}5,055 \\
4 \\
47 \\
4,404 \\
15 \\
55 \\
205 \\
326\end{array}$ \\
\hline
\end{tabular}

See footnotes at end of table. 
Table 18. Adjusted Sales of Kerosene by End Use, 1995 and 1996 (Continued) (Thousand Gallons)

\begin{tabular}{|c|c|c|c|c|c|c|}
\hline \multirow{2}{*}{ Destination } & \multicolumn{2}{|c|}{ Farm } & \multicolumn{2}{|c|}{ All Other } & \multicolumn{2}{|c|}{ Total } \\
\hline & 1995 & 1996 & 1995 & 1996 & 1995 & 1996 \\
\hline 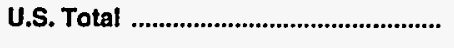 & 19,919 & 26,017 & 7,576 & 7,223 & 828,450 & 948,990 \\
\hline 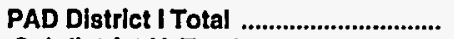 & 7,629 & 9,408 & 3,476 & 3,008 & 643,455 & 725,749 \\
\hline Subdistrict IA Total .............................. & 685 & 974 & 413 & 236 & 103,401 & 113,046 \\
\hline 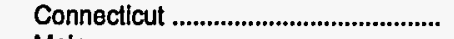 & 20 & 9 & 58 & - & 10,254 & 9,300 \\
\hline 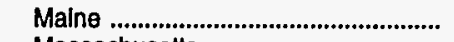 & 220 & 239 & 146 & 40 & 53,806 & 64,502 \\
\hline 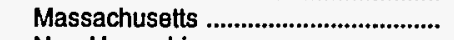 & 65 & 106 & 51 & 26 & 11,559 & 8,788 \\
\hline New Hampshire ................................... & 105 & 186 & 86 & 81 & 16,529 & 18,934 \\
\hline 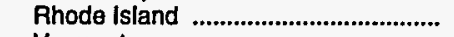 & - & - & - & 1 & 2,685 & 1,470 \\
\hline 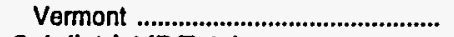 & 274 & 434 & 72 & 88 & 8,569 & 10,050 \\
\hline Subdistrict IB Total ........................... & 1,707 & 2,702 & 1,800 & 2,244 & 310,972 & 335,421 \\
\hline 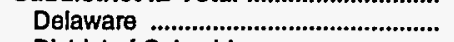 & 44 & 35 & 151 & 1 & 5,341 & 9,886 \\
\hline District of Columbia .............................. & - & - & - & - & 5,667 & 4,510 \\
\hline 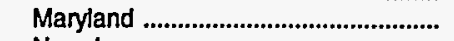 & 728 & 875 & 157 & 265 & 33,662 & 33,693 \\
\hline New Jersey ......................................... & 7 & 38 & 126 & 140 & 51,077 & 35,337 \\
\hline 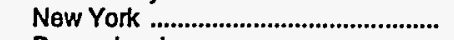 & 287 & 1,099 & 255 & 337 & 99,287 & 121,113 \\
\hline 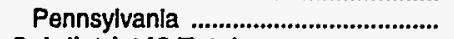 & 641 & 655 & 1,111 & 1,501 & 115,938 & 130,882 \\
\hline Subdlstrict IC Total ............................ & 5,238 & 5,732 & 1,262 & 528 & 229,082 & 277,282 \\
\hline Florida & 80 & 140 & 18 & 8 & 13,156 & 16,891 \\
\hline 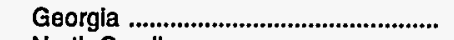 & 172 & 2 & 227 & 159 & 8,206 & 8,898 \\
\hline North Carolina ...................................... & 781 & 1,433 & 619 & 105 & 99,111 & 121,364 \\
\hline 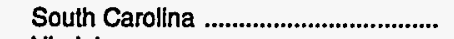 & 217 & 279 & 182 & 11 & 24,094 & 28,254 \\
\hline Virginia & 3,246 & 3,239 & 56 & 59 & 67,951 & 81,282 \\
\hline West Virginla ......................................... & 743 & 639 & 160 & 186 & 16,564 & 20,593 \\
\hline PAD Dlstrict II Total ................................ & 10,186 & 14,878 & 2,603 & 2,916 & 147,310 & 175,575 \\
\hline 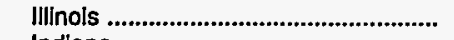 & 881 & 4,303 & 547 & 666 & 12,315 & 16,733 \\
\hline Indiana & 498 & 1,331 & 182 & 296 & 13,865 & 18,513 \\
\hline lowa & 1,499 & 691 & 37 & 23 & 2,879 & 2,262 \\
\hline 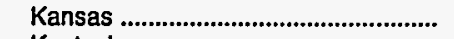 & 222 & 397 & 115 & 21 & 1,197 & 1,549 \\
\hline Kentucky ............................................... & 1,305 & 1,729 & 724 & 273 & 27,194 & 28,152 \\
\hline 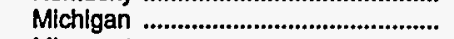 & 233 & 382 & 205 & 429 & 15,389 & 17,672 \\
\hline Minnesota ............................................ & 1,167 & 1,190 & 14 & 191 & 4,362 & 5,158 \\
\hline Mlssourf .............................................. & 237 & 302 & 95 & 49 & 2,231 & 4,892 \\
\hline 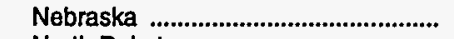 & 249 & 315 & 101 & 125 & 702 & 810 \\
\hline North Dakota ...................................... & 8 & 7 & 6 & 15 & 224 & 322 \\
\hline Ohlo & 3,161 & 3,488 & 350 & 592 & 43,025 & 50,167 \\
\hline Oklahoma .............................................. & 82 & 62 & 65 & 55 & 623 & 1,351 \\
\hline South Dakota ....................................... & 67 & 99 & 3 & 15 & 259 & 364 \\
\hline 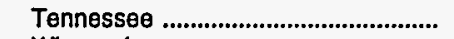 & 374 & 264 & 74 & 104 & 20,564 & 24,586 \\
\hline 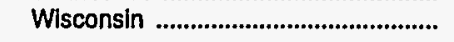 & 204 & 318 & 84 & 62 & 2,480 & 3,046 \\
\hline PAD District III Total .......................... & 425 & 516 & 837 & 517 & 19,149 & 21,185 \\
\hline 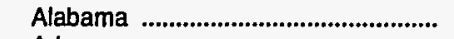 & 9 & 8 & 135 & 19 & 5,075 & 5,082 \\
\hline 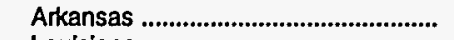 & 84 & 52 & 349 & 34 & 1,629 & 1,099 \\
\hline 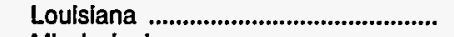 & 17 & 69 & 111 & 214 & 1,554 & 2,274 \\
\hline 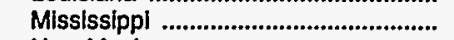 & 24 & 84 & 67 & 9 & 1,974 & 2,064 \\
\hline 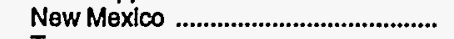 & 24 & 13 & 52 & 59 & 678 & 709 \\
\hline 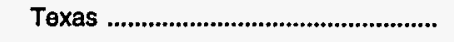 & 267 & 290 & 122 & 182 & 8,240 & 9,958 \\
\hline PAD Dlstrlct IV Total .......................... & 175 & 186 & 101 & 126 & 3,369 & 3,681 \\
\hline 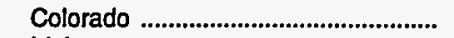 & 84 & 120 & 58 & 71 & 1,223 & 1,366 \\
\hline 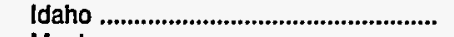 & 35 & 35 & 34 & 5 & 828 & 726 \\
\hline 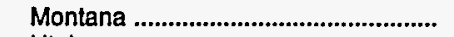 & 4 & 3 & - & 1 & 40 & 54 \\
\hline 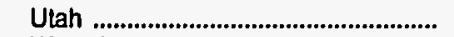 & 52 & 20 & 2 & 7 & 270 & 394 \\
\hline 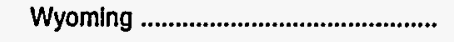 & - & 9 & 6 & 41 & 1,008 & 1,141 \\
\hline PAD Dlstrict V Total ............................ & 1,504 & 1,029 & 560 & 657 & 15,167 & 22,801 \\
\hline 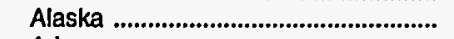 & - & - & - & 1 & 37 & 23 \\
\hline 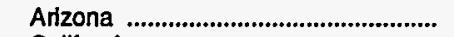 & 4 & 5 & 2 & 17 & 159 & 288 \\
\hline 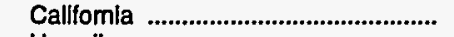 & 308 & 359 & 267 & 365 & 6,877 & 12,360 \\
\hline 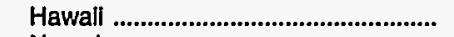 & 1 & 3 & - & - & 22 & 22 \\
\hline 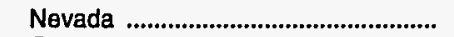 & 12 & 15 & 11 & 3 & 370 & 399 \\
\hline 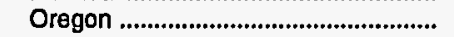 & 605 & 71 & 248 & 182 & 2,623 & 3,737 \\
\hline Washington .......................................... & 574 & 576 & 31 & 89 & 5,081 & 5,972 \\
\hline
\end{tabular}

Dashes $(-)=$ No data reported.

Note: Totals may not equal sum of components due to independent rounding.

Sources: - Energy Information Administration Form ElA-821, "Annual Fuel Oil and Kerosene Sales Report," for 1995 and 1996.

- Energy Informatlon Administration's "Petroleum Supply Annual," Volume 1, 1995 and 1996, (DOE/EIA-0340(95)/1 - (96)/1). 
Table 19. Adjusted Sales for Residential Use: Distillate Fuel Oil and Kerosene, 1996 (Thousand Gallons)

\begin{tabular}{|c|c|c|c|c|}
\hline \multirow{2}{*}{ Destination } & \multicolumn{3}{|c|}{ Distillate Fuel Oil } & \multirow{2}{*}{ Kerosene } \\
\hline & No. 1 & No. 2 & Total & \\
\hline 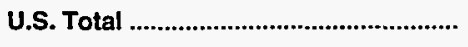 & 185,326 & $6,522,388$ & $6,707,714$ & 657,636 \\
\hline 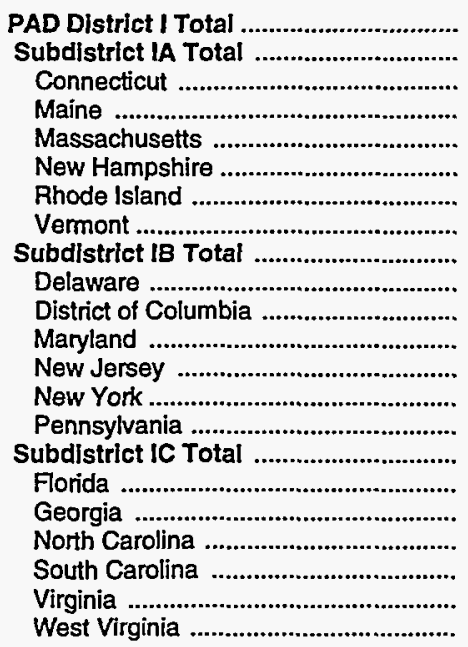 & $\begin{array}{r}31,859 \\
13,598 \\
2,428 \\
2,759 \\
7,533 \\
879 \\
- \\
- \\
15,165 \\
- \\
- \\
517 \\
54 \\
- \\
14,594 \\
3,096 \\
21 \\
64 \\
162 \\
1,063 \\
1,340 \\
446\end{array}$ & $\begin{array}{r}5,578,405 \\
2,100,664 \\
560,309 \\
319,007 \\
775,106 \\
197,011 \\
148,306 \\
100,925 \\
2,982,039 \\
46,513 \\
12,879 \\
247,186 \\
518,637 \\
1,288,927 \\
867,897 \\
495,702 \\
9,062 \\
6,366 \\
181,291 \\
29,281 \\
244,602 \\
25,101\end{array}$ & $\begin{array}{r}5,610,264 \\
2,114,262 \\
562,737 \\
321,766 \\
782,639 \\
197,890 \\
148,306 \\
100,925 \\
2,997,204 \\
46,513 \\
12,879 \\
247,703 \\
518,691 \\
1,288,927 \\
882,491 \\
498,798 \\
9,083 \\
6,430 \\
181,453 \\
30,344 \\
245,942 \\
25,547\end{array}$ & $\begin{array}{r}530,407 \\
95,280 \\
5,217 \\
57,548 \\
6,219 \\
16,486 \\
1,268 \\
8,542 \\
206,836 \\
7,574 \\
259 \\
24,925 \\
11,912 \\
60,898 \\
101,268 \\
228,291 \\
11,067 \\
6,047 \\
106,932 \\
23,570 \\
64,847 \\
15,829\end{array}$ \\
\hline 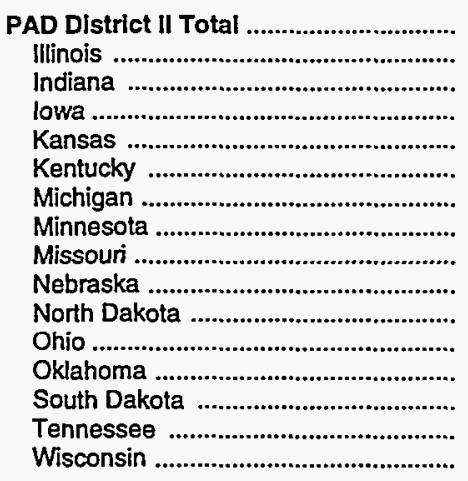 & $\begin{array}{r}107,786 \\
5,431 \\
9,885 \\
6,171 \\
401 \\
1,743 \\
14,521 \\
32,121 \\
2,289 \\
1,799 \\
7,252 \\
3,330 \\
828 \\
7,008 \\
1,011 \\
13,996\end{array}$ & $\begin{array}{r}777,453 \\
26,350 \\
51,770 \\
26,805 \\
339 \\
26,477 \\
149,868 \\
114,904 \\
11,790 \\
3,020 \\
27,590 \\
157,577 \\
164 \\
19,539 \\
10,434 \\
150,828\end{array}$ & $\begin{array}{r}885,239 \\
31,781 \\
61,655 \\
32,976 \\
740 \\
28,220 \\
164,389 \\
147,025 \\
14,079 \\
4,819 \\
34,842 \\
160,907 \\
992 \\
26,547 \\
11,445 \\
164,824\end{array}$ & $\begin{array}{r}107,822 \\
4,020 \\
12,096 \\
1,247 \\
808 \\
18,410 \\
9,646 \\
2,558 \\
2,368 \\
159 \\
224 \\
34,377 \\
843 \\
216 \\
19,139 \\
1,711\end{array}$ \\
\hline 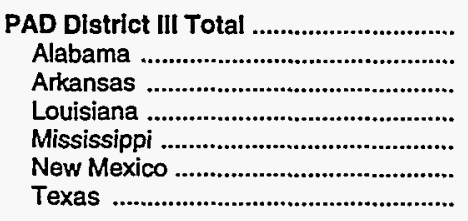 & $\begin{array}{r}81 \\
40 \\
- \\
20 \\
1 \\
20 \\
-\end{array}$ & $\begin{array}{r}475 \\
331 \\
33 \\
4 \\
22 \\
83 \\
3\end{array}$ & $\begin{array}{r}556 \\
371 \\
33 \\
24 \\
23 \\
103 \\
3\end{array}$ & $\begin{array}{r}6,706 \\
2,675 \\
493 \\
707 \\
941 \\
274 \\
1,616\end{array}$ \\
\hline 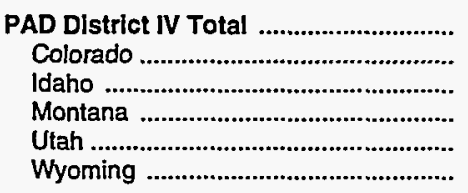 & $\begin{array}{r}17,584 \\
262 \\
4,055 \\
12,482 \\
465 \\
319\end{array}$ & $\begin{array}{r}31,249 \\
2,265 \\
18,057 \\
5,939 \\
3,752 \\
1,236\end{array}$ & $\begin{array}{r}48,833 \\
2,527 \\
22,112 \\
18,421 \\
4,217 \\
1,555\end{array}$ & $\begin{array}{r}1,626 \\
868 \\
526 \\
28 \\
176 \\
29\end{array}$ \\
\hline 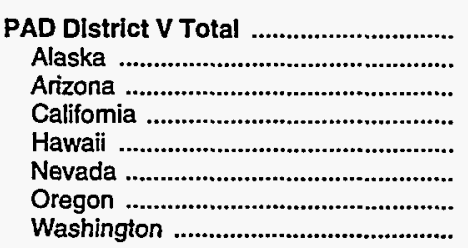 & $\begin{array}{r}28,015 \\
22,304 \\
20 \\
205 \\
- \\
279 \\
2,215 \\
2,994\end{array}$ & $\begin{array}{r}134,806 \\
32,837 \\
256 \\
4,022 \\
7 \\
5,396 \\
32,276 \\
60,012\end{array}$ & $\begin{array}{r}162,821 \\
55,141 \\
276 \\
4,227 \\
7 \\
5,675 \\
34,491 \\
63,006\end{array}$ & $\begin{array}{r}11,075 \\
12 \\
118 \\
4,343 \\
1 \\
263 \\
1,700 \\
4,640\end{array}$ \\
\hline
\end{tabular}

Dashes $(-)=$ No data reported.

Note: Totals may not equal sum of components due to independent rounding.

Sources: - Energy Information Administration Form ElA-821, "Annual Fuel Oil and Kerosene Sales Report," for 1996.

- Energy Information Administration's "Petroleum Supply Annual," Volume 1, 1996, (DOE/EIA-0340(96)/1). 
Table 20. Adjusted Sales for Commercial Use: Distillate Fuel Oil, Residual Fuel Oil, and Kerosene, 1996

(Thousand Gallons)

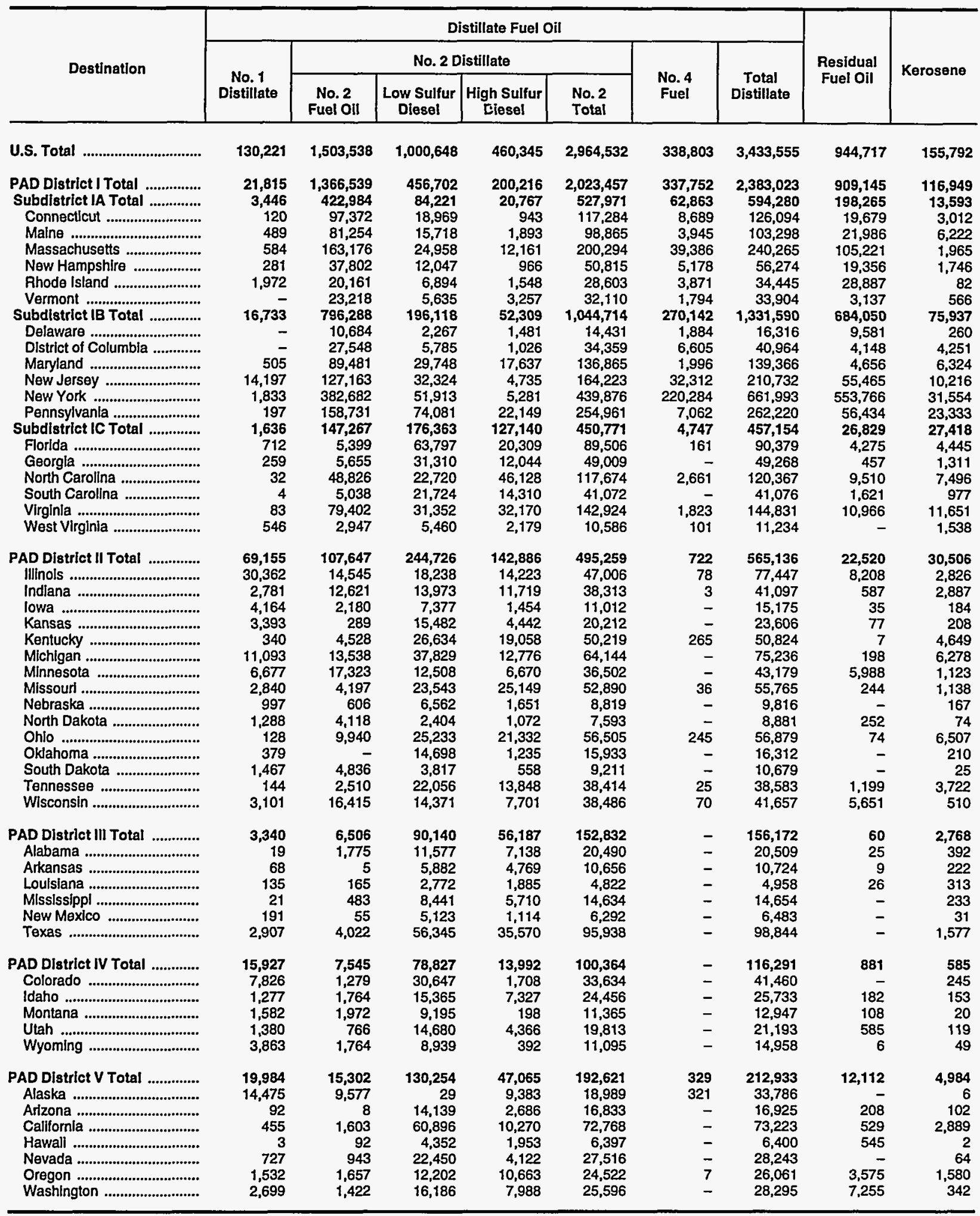

Dashes $(-)=$ No data reported.

Note: Totals may not equal sum of components due to independent rounding

Sources: - Energy Information Administration Form EIA-821, "Annual Fuel Oil and Kerosene Sales Report," for 1996.

- Kerosene data are based on data from the Energy Information Administration's "Petroleum Supply Annual," Volume 1, 1996, (DOE/EIA-0340(96)/1). 
Table 21. Adjusted Sales for Industrial Use: Distillate Fuel Oil, Residual Fuel Oil, and Kerosene, 1996

(Thousand Gallons)

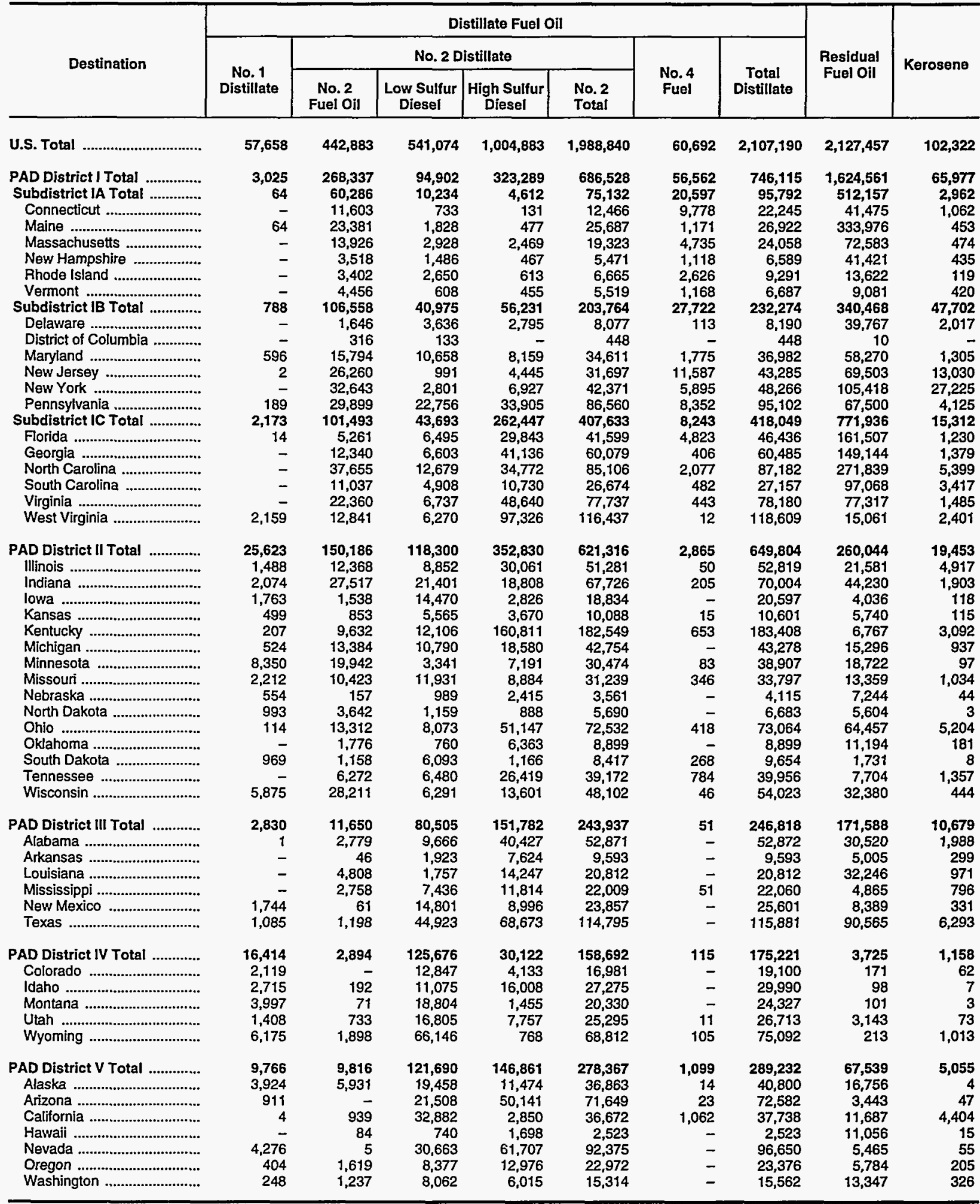

Dashes $(-)=$ No data reported.

Note: Totals may not equal sum of components due to independent rounding.

Sources: - Energy Information Administration Form ElA-821, "Annual Fuel Oil and Kerosene Sales Report," for 1996.

- Kerosene data are based on data from the Energy Information Administration's "Petroleum Supply Annual," Volume 1, 1996, (DOEJEIA-0340(96)/1). 
Table 22. Adjusted Sales for Farm Use: Distillate Fuel Oil and Kerosene; Sales for Electric Utility and Oil Company Uses: Distillate Fuel Oil and Residual Fuel Oil, 1996 (Thousand Gallons)

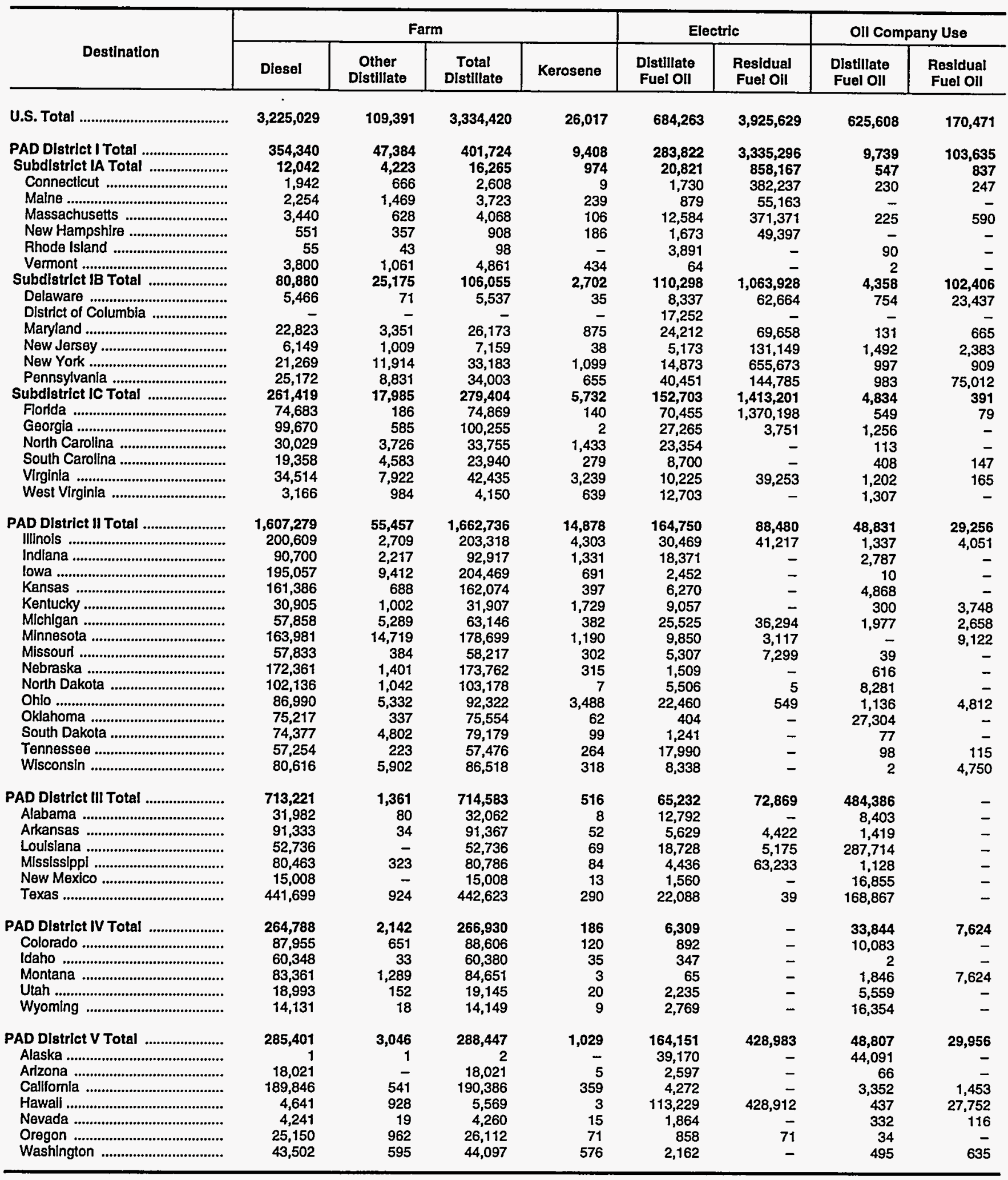

Dashes $(-)=$ No data reported.

Note: Totals may not equal sum of components due to independent rounding.

Sources: - Energy Information Administration Form ElA-821, "Annual Fuel Oil and Kerosene Sales Report," for 1996.

- Kerosene data are based on data from the Energy Information Administration's "Petroleum Supply Annual," Volume 1, 1996, (DOE/EIA-0340(96)/1). 
Table 23. Adjusted Sales for Transportation Use: Distillate Fuel Oil and Residual Fuel Oil, 1996 (Thousand Gallons)



E Estimated data. Florida's 1996 estimate is based on seven months of data. Nevada's 1996 estimate is based on eight months of data. Connectlcut's 1996 data are a Federal Highway Administration estimate.

Dashes $(-)=$ No data reported.

Note: Totals may not equal sum of components due to independent rounding.

Sources: • Energy Information Administration Form ElA-821, "Annual Fuel Oil and Kerosene Sales Report," for 1996.

- On-Highway Diesel data are Federal Highway Administation statistics of highway special fuels use.

- Energy Information Administration's "Petroleum Supply Annual," Volume 1, 1996, (DOE/EIA-0340(96)/1). 
Table 24. Adjusted Sales for Military, Off-Highway, and All Other Uses: Distillate Fuel Oil, Residual Fuel Oil, and Kerosene, 1996

(Thousand Gallons)

\begin{tabular}{|c|c|c|c|c|c|c|c|c|c|c|}
\hline \multirow{2}{*}{ DestInation } & \multicolumn{4}{|c|}{ Milltary } & \multicolumn{3}{|c|}{$\begin{array}{l}\text { Off-Highway } \\
\text { Distillate Fuel OII }\end{array}$} & \multicolumn{3}{|c|}{ All Other } \\
\hline & Dlesel & $\begin{array}{c}\text { Other } \\
\text { Distlllate }\end{array}$ & $\begin{array}{c}\text { Total } \\
\text { Distillate }\end{array}$ & $\begin{array}{l}\text { Residual } \\
\text { Fuel OIl }\end{array}$ & Construction & Other & Total & $\begin{array}{l}\text { Distillate } \\
\text { Fuel Oll }\end{array}$ & $\begin{array}{l}\text { Residual } \\
\text { Fuel Oll }\end{array}$ & Kerosene \\
\hline U.S. Total & 323,436 & 47,657 & 371,093 & 57,249 & $1,645,647$ & 417,890 & $2,063,538$ & - & 10,542 & 7,223 \\
\hline PAD Dlstrlct I Total ................. & 41,266 & 38,874 & 80,141 & 40,120 & 504,241 & 108,581 & 612,822 & - & 7,792 & 3,008 \\
\hline Subdlstrict IA Total ............... & 4,341 & 6,722 & 11,062 & 11,212 & 51,202 & 22,800 & 74,001 & - & 354 & 236 \\
\hline Connecticut .................................. & 254 & 3,615 & 3,869 & 1,376 & 7,698 & 1,774 & 9,472 & - & - & \\
\hline 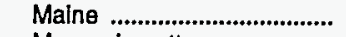 & 593 & 2,937 & 3,530 & 6,676 & 12,131 & 14,149 & 26,279 & - & 354 & 40 \\
\hline 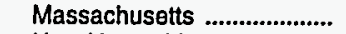 & 1,378 & 53 & 1,431 & 3,160 & 22,549 & 1,060 & 23,609 & - & - & 26 \\
\hline New Hampshire ..................... & 52 & - & 52 & 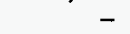 & 4,163 & 5,103 & 9,266 & - & - & 81 \\
\hline 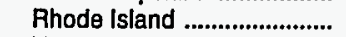 & 1,866 & 30 & 1,895 & - & 2,756 & 276 & 3,032 & - & - & 1 \\
\hline 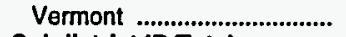 & 198 & 88 & 286 & - & 1,905 & 437 & 2,342 & - & - & 88 \\
\hline Subdistrict iB Total ................ & 12,996 & 24,198 & 37,195 & 14,018 & 158,460 & 11,207 & 169,667 & - & - & 2,244 \\
\hline Delaware ..................................... & 286 & 1,042 & 1,328 & 806 & 6,763 & 216 & 6,979 & - & - & 1 \\
\hline District of Columbia .............. & 73 & 1,935 & 2,008 & - & 320 & - & 320 & - & - & - \\
\hline 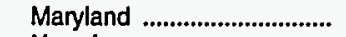 & 9,124 & 8,890 & 18,014 & 6,731 & 23,364 & 1,040 & 24,404 & - & - & 265 \\
\hline 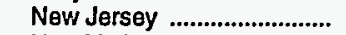 & 1,282 & 652 & 1,934 & 4,289 & 26,573 & 3,614 & 30,187 & - & - & 140 \\
\hline 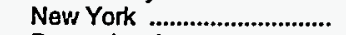 & 231 & 2,123 & 2,354 & 890 & 47,508 & 177 & 47,685 & - & - & 337 \\
\hline 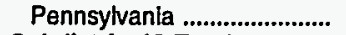 & 2,000 & 9,556 & 11,556 & 1,302 & 53,933 & 6,160 & 60,093 & - & - & 1,501 \\
\hline Subdlatrict IC Total ............... & 23,930 & 7,954 & 31,883 & 14,890 & 294,579 & 74,575 & 369,153 & - & 7,438 & 528 \\
\hline 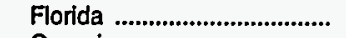 & 7,268 & - & 7,268 & 1,581 & 111,351 & 7,580 & 118,931 & - & 7,385 & 8 \\
\hline 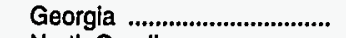 & 2,553 & 425 & 2,978 & - & 50,376 & 21,365 & 71,741 & - & - & 159 \\
\hline 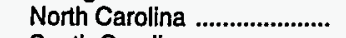 & 2,870 & 999 & 3,869 & 6,635 & 56,304 & 8,985 & 65,288 & - & 53 & 105 \\
\hline 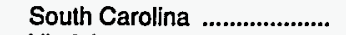 & 1,273 & 670 & 1,943 & 2,920 & 20,449 & 18,600 & 39,049 & - & - & 11 \\
\hline 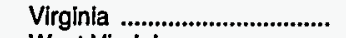 & 9,769 & 5,491 & 15,260 & 3,754 & 47,930 & 16,369 & 64,299 & - & - & 59 \\
\hline 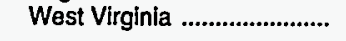 & 196 & 368 & 564 & - & 8,169 & 1,676 & 9,845 & - & - & 186 \\
\hline PAD District \|l Total .................. & 18,700 & 3,126 & 21,826 & 657 & 535,509 & 100,734 & 636,243 & - & - & 2,916 \\
\hline 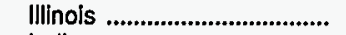 & 1,252 & 148 & 1,400 & 21 & 64,543 & 5,626 & 70,169 & - & - & 666 \\
\hline 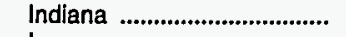 & 1,653 & 203 & 1,856 & - & 23,828 & 9,455 & 33,283 & - & - & 296 \\
\hline 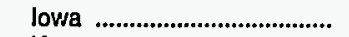 & 223 & - & 223 & - & 30,485 & 10,599 & 41,085 & - & - & 23 \\
\hline 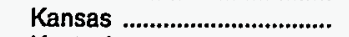 & 924 & - & 924 & - & 27,456 & 553 & 28,009 & - & - & 21 \\
\hline 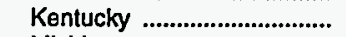 & 1,380 & - & 1,380 & - & 37,613 & 6,510 & 44,123 & - & - & 273 \\
\hline 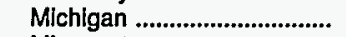 & 393 & 1,202 & 1,595 & 122 & 43,109 & 14,186 & 57,295 & - & - & 429 \\
\hline 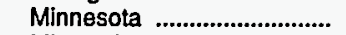 & 670 & - & 670 & - & 44,107 & 15,637 & 59,743 & - & - & 191 \\
\hline 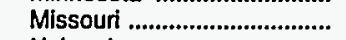 & 2,101 & 645 & 2,747 & 514 & 36,659 & 6,801 & 43,460 & - & - & 49 \\
\hline 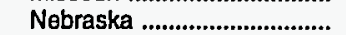 & 465 & - & 465 & - & 15,916 & 1,736 & 17,652 & - & - & 125 \\
\hline North Dakota ............................. & 1.713 & 124 & 1,837 & - & 5,075 & 835 & 5,911 & - & - & 15 \\
\hline 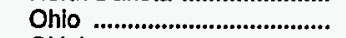 & 750 & 5 & 755 & - & 66,953 & 5,471 & 72,424 & - & - & 592 \\
\hline 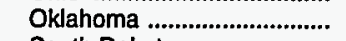 & 1,758 & - & 1,758 & - & 29,210 & 3,377 & 32,587 & - & - & 55 \\
\hline 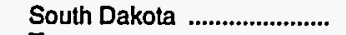 & 889 & 789 & 1,677 & - & 7,037 & 1,379 & 8,416 & - & - & 15 \\
\hline 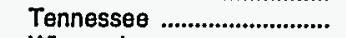 & 1,375 & - & 1,375 & - & 53,620 & 7,872 & 61,492 & - & - & 104 \\
\hline 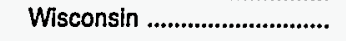 & 3,155 & 11 & 3,165 & - & 49,897 & 10,698 & 60,595 & - & - & 62 \\
\hline PAD District III Total .............. & 157,950 & 704 & 158,654 & 4,767 & 297,024 & 104,173 & 401,197 & - & 31 & 517 \\
\hline 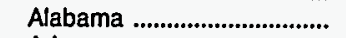 & 2,622 & - & 2,622 & - & 57,678 & 36,612 & 94,290 & - & - & 19 \\
\hline 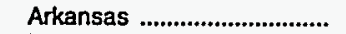 & 721 & 704 & 1,425 & - & 12,768 & 10,012 & 22,780 & - & - & 34 \\
\hline 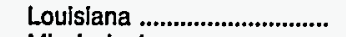 & 34,426 & - & 34,426 & 1,881 & 93,233 & 7,514 & 100,748 & - & - & 214 \\
\hline 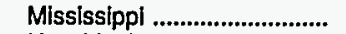 & 3,796 & - & 3,796 & - & 15,214 & 23,124 & 38,337 & - & - & 9 \\
\hline 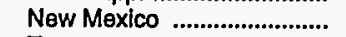 & 1,845 & - & 1,845 & - & 15,189 & 1,992 & 17,181 & - & 31 & 59 \\
\hline Texas & 114,539 & - & 114,539 & 2,886 & 102,941 & 24,919 & 127,861 & - & - & 182 \\
\hline PAD Dlstrict IV Total ................. & 2,344 & 3,444 & 5,788 & 8 & 137,865 & 33,575 & 171,440 & - & 71 & 126 \\
\hline Colorado ........................................ & 1,020 & 93 & 1,113 & 8 & 53,018 & 2,292 & 55,310 & - & 4 & 71 \\
\hline 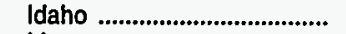 & 673 & - & 673 & - & 16,344 & 16,080 & 32,424 & - & 5 & 5 \\
\hline 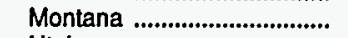 & 269 & - & 269 & - & 28,825 & 5,776 & 34,602 & - & - & 1 \\
\hline 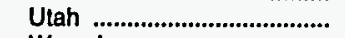 & 356 & 3,351 & 3,706 & - & 21,543 & 4,052 & 25,595 & - & 24 & 7 \\
\hline 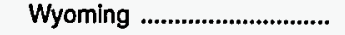 & 26 & - & 26 & - & 18,134 & 5,375 & 23,509 & - & 38 & 41 \\
\hline PAD District V Total ................ & 103,175 & 1,509 & 104,684 & 11,697 & 171,009 & 70,826 & 241,836 & - & 2,649 & 657 \\
\hline Alaska & 3,164 & 448 & 3,612 & - & 10,502 & 11,390 & 21,892 & - & - & 1 \\
\hline 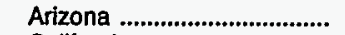 & 700 & 9 & 709 & - & 23,423 & 2,238 & 25,661 & - & - & 17 \\
\hline 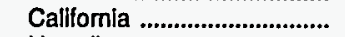 & 62,040 & 106 & 62,147 & 7,509 & 84,317 & 23,659 & 107,976 & - & 28 & 365 \\
\hline 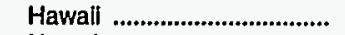 & 12,912 & - & 12,912 & 3,460 & 4,694 & 373 & 5,067 & - & 2,621 & - \\
\hline 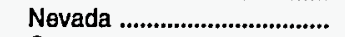 & 967 & - & 967 & - & 10,902 & 1,114 & 12,016 & - & - & 3 \\
\hline 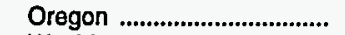 & 1,510 & 39 & 1,548 & - & 9,274 & 14,249 & 23,523 & - & - & 182 \\
\hline 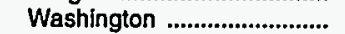 & 21,883 & 907 & 22,790 & 729 & 27,898 & 17,803 & 45,702 & - & - & 89 \\
\hline
\end{tabular}

Dashes $(-)=$ No data reported.

Note: Totals may not equal sum of components due to independent rounding.

Sources: - Energy Information Administration Form ElA-821, "Annual Fuel Oil and Kerosene Sales Report," for 1996.

- Energy Information Administration's "Petroleum Supply Annual," Volume 1, 1996, (DOE/EIA-0340(96)/1). 
Appendix A

Technical Notes

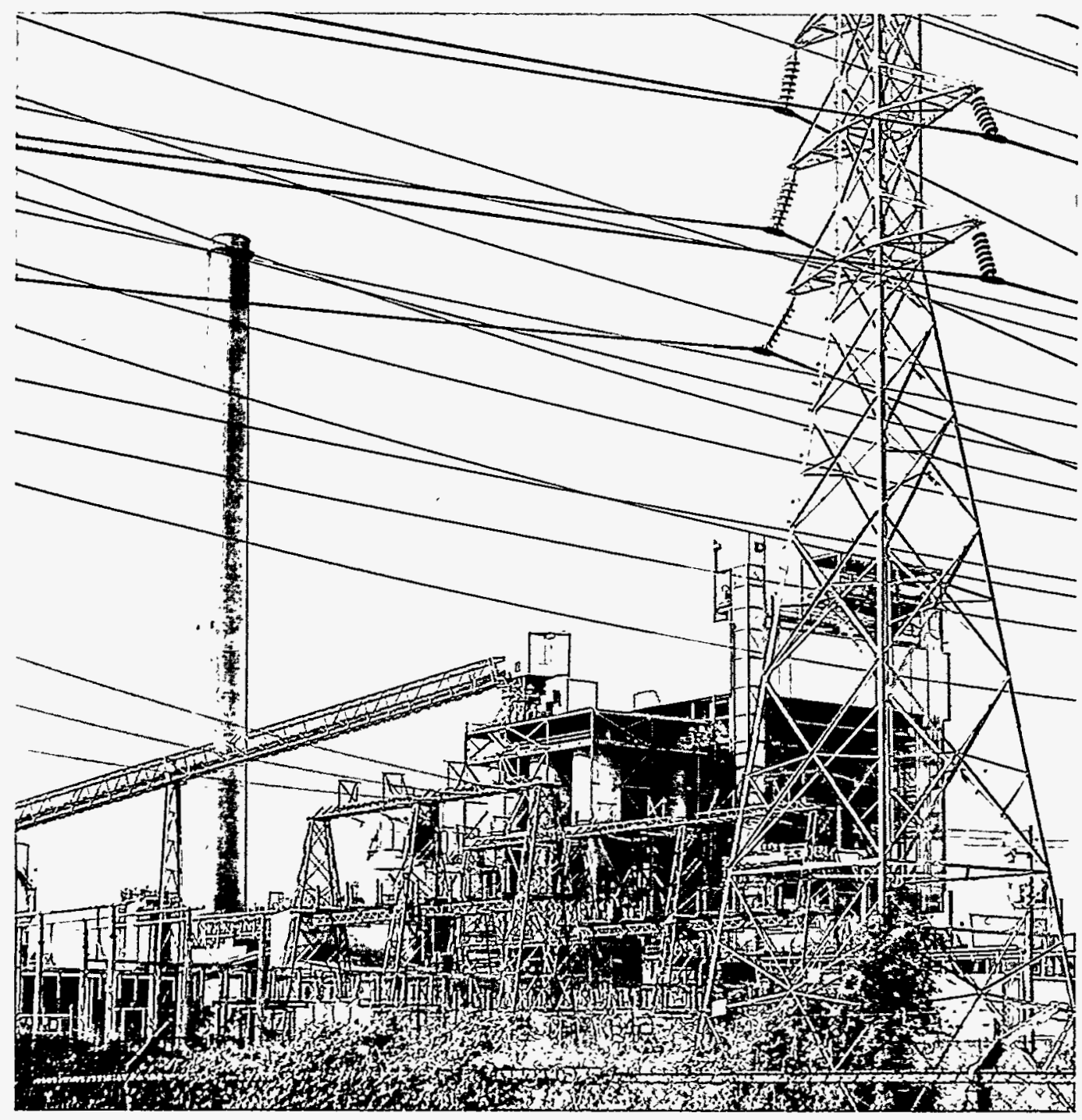

Plants burning fuel oil or kerosene to generate electricity are classified as electric utility use. 



\section{Appendix A}

\section{Technical Notes}

\section{Note 1: EIA-821: Annual Fuel Oil and Kerosene Sales Report, 1996}

\section{Background}

The EIA-821, "Annual Fuel Oil and Kerosene Sales Report" survey was implemented to meet Energy Information Administration (EIA) legislative mandates and data user needs. Form EIA-821 is used to gather data on the sales to end users of distillate fuel oil, residual fuel oil, and kerosene. The data are used to determine the level of demand by end-use category and product at the State, regional, and national levels. This mandatory report is authorized by the Federal Energy Administration Act of 1974 (P. L. 93-275).

The predecessor survey to the EIA-821 was the EIA-172, "Sales Report of Fuel Oil and Kerosene" survey. The EIA-172 was conducted for reference years 1979 through 1982. Due to a serious deterioration in the sample of respondents during the 4-year period, the Form EIA-172 data were not collected for 1983. Instead, estimates for 1983 were published and a new sample of respondents was designed and selected from an improved sampling frame. The EIA-821 survey commenced with reference year 1984.

\section{Discussion of Sampling Frame}

The target population for the fuel oil and kerosene sales survey is the universe of companies that sell fuel oil and kerosene to end users.

The EIA-863 data base provided the sampling frame for the 1996 EIA-821 survey. The EIA-863, "Petroleum Product Sales Identification Survey," was mailed to approximately 30,000 companies in January 1995 to collect 1994 State-level sales volume data for No. 2 distillate, residual fuel, motor gasoline, and propane. Companies also indicated if they sold kerosene. The No. 2 distillate data were further identified by residential and nonresidential end use, and non-end-use sales; the residual data were identified by end use and nonend-use; motor gasoline was identified by end use and non-end use and by conventional and oxygenated and/or reformulated; and propane was identified by residential, nonresidential, and non-end-use sales. The mailing list for the EIA-863 survey was constructed by merging and unduplicating the 1991 frame file and more than 50 State and commercial lists. In addition, company/ State-level volumes for distillate fuel, residual fuel, motor gasoline, and propane from the 1994 EIA-821 survey were merged with the EIA-863 to yield a combined data file. The transformed and edited version of the frame was then used to design and select the 1995 EIA-821 sample.

It should be noted that truck stops selling No. 2 diesel fuel were not specifically included in the EIA-863 survey. Therefore, the EIA-821 survey does not include all sales of No. 2 distillate through retail truck stops. However, to compensate for this shortage in coverage, data from the Federal Highway Administration are presented in the tables for on-highway diesel sales.

The sampling frame also lacked full coverage of kerosene retailers, many of whom are hardware stores and service stations. To account for all kerosene volumes supplied to the marketplace, aggregate reported kerosene volumes are set equal to the products supplied volumes published in the Petroleum Supply Annual.

\section{Discussion of the Sample Design}

The purpose of the EIA-821 sample design was to produce State-level estimates of total sales for the following five target variables: (1) residential No. 2 distillate, (2) other retail No. 2 distillate, (3) wholesale No. 2 distillate, (4) retail residual, and (5) wholesale residual. A separate sample was initially designed for each target variable.

Companies were divided into two basic types of strata: certainty and noncertainty. Companies selected with certainty were:

- all refiners and gas plant operators

- companies doing business in four or more States

- companies accounting for 5 percent or more of the distillate or residual fuel oil volume for any target variable or particular end-use category sold in a State.

- companies accounting for 5 percent or more of the kerosene volume sold in a State in an earlier EIA-821 survey. 
- augmented frame units, e.g., vessel bunkering, for which no attribute data were available.

Firms determined not to be selling distillate or residual fuel oil or kerosene were removed from the frame file. Volumes for nonrespondents to the EIA-863 who had reported in the previous frame survey or in a sample survey were imputed. The imputed nonrespondents and the noncertainty companies were stratified by sales volume for each target variable. Strata boundaries were determined using the Dalenius-Hodges procedures, allowing the number of strata and the strata boundaries to vary by State. Nonrespondents for whom no previous information was available were classified in a separate stratum and sampled with reduced probabilities of selection.

Neyman allocation was used to allocate the number of companies required for each strata to obtain the required levels of accuracy: a 2.5 percent coefficient of variation for residual fuel oil and a 5 percent coefficient of variation for distillate fuel oil. Sample selections were then performed simultaneously from the same random list for the five samples to produce a final linked sample of approximately 4,700 companies. This method reduced the total survey sample size while improving volume estimates. Selected companies were asked to report sales by end-use categories for distillate and residual fuel oil and kerosene.

\section{Imputation and Estimation}

Survey data gathered from the respondents may contain incomplete reporting, total nonresponse, or values that fail editing. Missing data are estimated, or implicitly imputed for, using the strata means and treated the same as reported data. The weighted estimate is defined as follows:

$\hat{V}=\Sigma_{h} N_{h}\left(\Sigma_{i} W_{h i} V_{h i} / \Sigma_{i} W_{h i}\right)$

where:

$\hat{V} \quad=$ total estimated volume

$\Sigma_{h}=$ denotes summation over strata

$N_{h}=$ stratum population for stratum $\mathrm{h}$

$\Sigma_{i}=$ denotes summation over units within stratum $\mathrm{h}$

$V_{h} i=$ volume reported for unit $\mathrm{i}$ in stratum $\mathrm{h}$

$W_{h i}=$ weight attached to unit $\mathrm{i}$ in stratum $\mathrm{h}$.

where: $W_{h i}$ is inversely proportional to the probability of inclusion in the linked sample.
The degree of imputation by product and end use at the U.S. total level ranged as follows for the 1996 EIA-821 data: distillate, 0.07 percent for oil company use to 15.8 percent for off-highway use; residual fuel oil, 0.01 percent for all other uses to 3.0 percent for military use; and kerosene, 0.7 percent for farm use to 4.1 percent for residential use.

\section{Collection Methods}

The EIA-821 form was mailed in January 1997 to all companies selected for participation in the 1996 reference year survey. The completed form was due to EIA on March 3, 1997. A second request was sent by certified mail to nonrespondents approximately 1 month after the filing deadline. Follow-up telephone calls were made to collect outstanding data and to verify reported data. Late submissions and resubmissions were processed when received.

\section{Data Processing}

As EIA-821 forms were received, they were logged into an automated Survey Control File that maintains company identification and survey form status information for each company selected to participate in the survey. The data were reviewed manually, entered onto the computer files, and then processed through an automated edit program which detected missing data and outlying values. Data that failed the edits were resolved through telephone calls to the data reporters and corrections were entered onto the computer files. Preliminary estimates were generated and processed through a series of validation procedures to flag and rectify potential misreporting of data. Statistical reports, including publication tables, were produced using only acceptable and verified data.

\section{Note 2: Reliability of the Data}

Two types of errors are associated with data produced from a sample survey--sampling errors and nonsampling errors. Sampling errors occur because the estimates are based on a sample rather than on a census. The particular sample used for the EIA-821 survey is one of a large number of samples of equal size which could have been selected from the sampling frame using the same sample design. Each of these samples would produce a different estimate. If the estimates were averaged over all possible samples, the result would be the same as the estimate derived from a census of the sampling frame. The sampling error is a measure of variability among the estimates from all possible samples and, thus, is a measure of the 
precision with which an estimate from a particular sample approximates the results of a census.

Nonsampling errors and possibly biases can arise from a number of sources: (1) inability to obtain information about all cases in the sample, (2) response errors, (3) differences in the interpretation of questions or definitions, (4) mistakes in recording or coding of the data obtained, and (5) other errors of collection, response, coverage, and estimation for missing data. Bias is the difference between the average of the estimates over all possible samples of the same size and design, and the true value being estimated. It is not possible to estimate bias using the results of one sample.

Data obtained from alternate sources are not subject to sampling errors, but may be subject to nonsampling errors, the magnitudes of which are unknown. Nonsampling errors for survey estimates and estimates adjusted to alternate sources cannot be determined, but attempts are made throughout survey processing to minimize this type of error.

Data in Tables 1 through 12 are based on survey data which are subject to sampling errors. Coefficients of variation, which are estimates of sampling errors, are presented for the retail target variables in the following table for the 1995 survey. The coefficients of variation (CV) were estimated by:

$C V(\hat{V})=\frac{\sqrt{V A R(\hat{V})}}{\hat{V}}$ where:

$\operatorname{VAR}(\hat{V})=\sum_{h} n_{h}\left(1-\frac{n_{h}}{N_{h}}\right) S_{h}^{2}$

$S_{h}^{2}=\frac{\sum_{i=1}^{n h} W_{i}^{2} V_{i}^{2}+\bar{V}_{h}^{2} \sum_{i=1}^{n h} W_{i}^{2}-2 \bar{V}_{h} \sum_{i=1}^{n h} W_{i}^{2} V_{i}}{n_{h}-1}$

$\bar{V}_{h}=\frac{\sum_{i=1}^{n_{h}} W_{i} V_{i}}{\sum_{i=1}^{n h} W_{i}}$

$\hat{V}=$ total estimated volume,

$N_{h}=$ stratum population for stratum h,

$n_{h}=$ number of sample units in stratum $\mathrm{h}$,

$V_{i}=$ volume for unit $\mathrm{i}$,

$W_{i}=$ weight for unit $i$.

Response rates also offer some indication of the reliability and comprehensiveness of survey results. For the 1996 EIA-821 survey, the overall response rate (the number of submissions received, divided by the number of submissions solicited and expected, times 100) was 93.1 percent. 
Coefficients of Variation for Sales of Fuel Oil, 1996

\begin{tabular}{|c|c|c|c|}
\hline Geographic Area & $\begin{array}{l}\text { Residential Distillate } \\
\text { Fuel Oil }\end{array}$ & $\begin{array}{l}\text { Non-Residential } \\
\text { Retail Distillate } \\
\text { Fuel Oil }\end{array}$ & $\begin{array}{c}\text { Retail } \\
\text { Residual Fuel Oil }\end{array}$ \\
\hline U.S. Average & 1.7 & 2.8 & 0.1 \\
\hline PAD District I Average ........................... & 2.0 & 1.4 & 0.1 \\
\hline 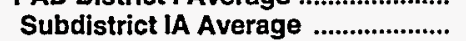 & 3.2 & 1.8 & 0.1 \\
\hline 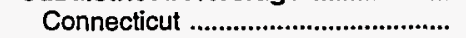 & 4.7 & 3.5 & 0.0 \\
\hline 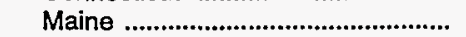 & 13.2 & 3.9 & 0.0 \\
\hline 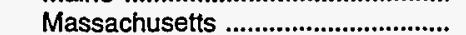 & 5.4 & 3.6 & 0.1 \\
\hline 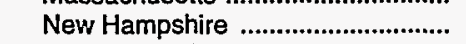 & 6.4 & 3.5 & 0.0 \\
\hline 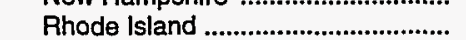 & 5.5 & 5.7 & 0.0 \\
\hline Vermont & 3.2 & 3.8 & 0.0 \\
\hline Subdistrict lB Average .................... & 3.0 & 2.6 & 0.1 \\
\hline Delaware & 3.2 & 5.9 & 0.0 \\
\hline 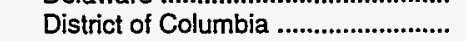 & 0.6 & 0.3 & 0.0 \\
\hline 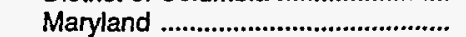 & 3.7 & 3.4 & 0.0 \\
\hline New Jersey ....................................... & 4.7 & 3.6 & 0.0 \\
\hline 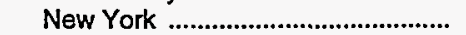 & 5.8 & 6.3 & 0.1 \\
\hline 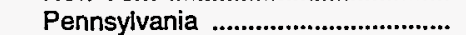 & 5.0 & 4.4 & 0.0 \\
\hline 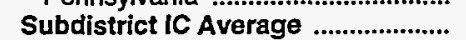 & 3.3 & 2.0 & 0.1 \\
\hline 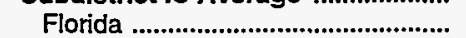 & 10.7 & 2.6 & 0.0 \\
\hline 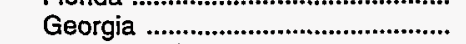 & 15.3 & 4.2 & 0.0 \\
\hline 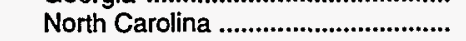 & 5.0 & 6.3 & 0.3 \\
\hline 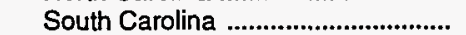 & 6.4 & 7.4 & 0.0 \\
\hline Virginia & 5.5 & 5.1 & 0.0 \\
\hline 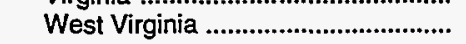 & 7.8 & 3.0 & 0.0 \\
\hline PAD District Il Average ........................ & 2.9 & 1.3 & 0.1 \\
\hline 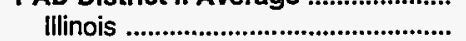 & 6.5 & 6.7 & 0.0 \\
\hline 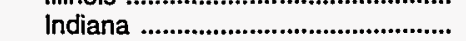 & 6.4 & 2.6 & 0.0 \\
\hline lowa & 8.8 & 3.9 & 0.0 \\
\hline 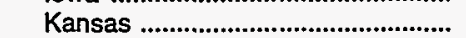 & 15.8 & 6.9 & 1.3 \\
\hline Kentucky .......................................... & 7.4 & 3.2 & 0.0 \\
\hline 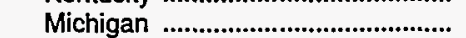 & 7.4 & 4.2 & 0.0 \\
\hline 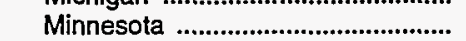 & 8.1 & 7.8 & 0.0 \\
\hline Missoun & 5.8 & 4.6 & 0.0 \\
\hline 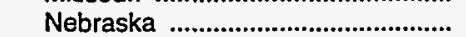 & 15.3 & 3.8 & 0.0 \\
\hline 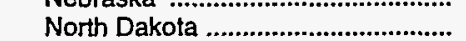 & 7.7 & 3.8 & 0.0 \\
\hline & 7.3 & 2.9 & 0.0 \\
\hline Oklahoma & 62.3 & 5.8 & 0.0 \\
\hline South Dakota & 9.8 & 4.5 & 0.0 \\
\hline 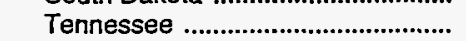 & 17.4 & 2.5 & 0.0 \\
\hline 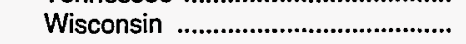 & 7.9 & 4.4 & 0.0 \\
\hline PAD District III Average ....................... & 5.9 & 13.1 & 0.1 \\
\hline Alabama & 7.6 & 6.2 & 0.1 \\
\hline Arkansas & 42.3 & 4.1 & 0.0 \\
\hline 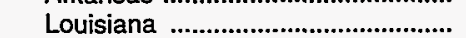 & 41.0 & 8.6 & 0.0 \\
\hline 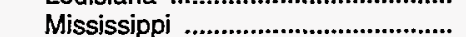 & 4.5 & 4.7 & 0.0 \\
\hline New Mexico & 2.8 & 4.5 & 0.0 \\
\hline Texas & 0.0 & 23.6 & 0.0 \\
\hline PAD District IV Average ..................... & 14.8 & 1.8 & 0.1 \\
\hline Colorado & 66.4 & 3.5 & 0.0 \\
\hline ldaho & 10.7 & 4.0 & 0.0 \\
\hline Montana & 35.7 & 6.6 & 0.1 \\
\hline Utah & 14.1 & 4.4 & 0.0 \\
\hline 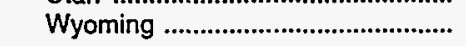 & 5.2 & 2.7 & 0.0 \\
\hline PAD District $V$ Average ...................... & 2.0 & 2.2 & 0.1 \\
\hline Alaska & 2.9 & 1.4 & 0.0 \\
\hline Arizona & 22.4 & 3.0 & 0.0 \\
\hline 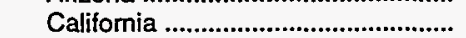 & 9.3 & 4.5 & 0.0 \\
\hline 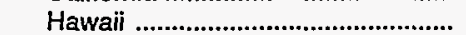 & 0.0 & 4.1 & 0.0 \\
\hline Nevada .......................................... & 1.7 & 3.9 & 0.0 \\
\hline 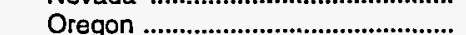 & 4.3 & 4.0 & 0.0 \\
\hline Washington & 3.6 & 4.2 & 0.1 \\
\hline
\end{tabular}

Note: Coefficients of variation that are less than 0.1 and greater than zero are displayed as 0.1 .

Sources: • Energy Information Administration Form EIA-821, "Annual Fuel Oil and Kerosene Sales Report," for 1996. 


\section{Note 3: Data Adjustments}

\section{Alternate Source Data}

After all preliminary tabulations were verified, comparisoms were made between the survey results and available alternate source data. The following end-use categories were replaced by alternate source data at the U.S., Petroleum Administration for Defense (PAD) district, or State level:

\section{Tables 1 through 12:}

On-Highway Diesel. Distillate fuel oil by State was calculated from the Federal Highway Administration data on highway use of special fuels. Of the 1992 through 1996 special fuels, more than 98 percent is diesel.

\section{Tables 13 through 24:}

Electric Utility. National-level distillate and residual fuel oil sales for electric utility use were calculated from annual aggregations of data collected on forms EIA-759, "Monthly Power Plant Report," and FERC-423, "Monthly Report of Cost and Quality of Fuels for Electric Plants." Form EIA-759 consumption data for light oils include No. 2 fuel oil, kerosene, and jet fuel; the heavy oils include No. 4 fuel oil, residual fuel oil, and crude oil. Using receipt data from Form FERC-423, the No. 4 fuel oil receipts were deleted from the heavy oil consumption data and added to the light oil consumption data. The resultant distillate (light oils) and residual (heavy oils) consumption data were added to the stock change of light oils and heavy oils, respectively, from Form EIA-759 to obtain the estimate of sales. Allocations at the State level were based on the EIA-821 survey.

On-Highway Diesel. Distillate fuel oil by State was calculated from the Federal Highway Administration data on highway use of special fuels. Of the 1992 through 1996 special fuels, more than 98 percent is diesel.

\section{2 through 1996 Adjustments}

Kerosene volumes in Tables 1 through 24 are adjusted at the national level to equal products supplied volumes as published in the "Detailed Statistics" section of the Petroleum Supply Annual (PSA). The 1996 survey resulted in 812,140 thousand gallons of kerosene at the national level. The products supplied volume at the national level for kerosene was 948,990 thousand gallons. Hence, the adjustment factor at the national level for 1996 was 1.17 .

Volumes of distillate fuel oil in Tables 13 through 24 were adjusted at the PAD district level to equal products supplied volumes. The adjustment factors at the PAD district level for 1996 are as follows:

\section{Distillate Fuel Oil \\ - PAD District I: $\quad .98$ \\ - PAD District II: $\quad .98$ \\ - PAD District III: $\quad .85$ \\ - PAD District IV: 1.30 \\ - PAD District V: $\quad .66$}

For Tables 13 through 24 , total sales at the PAD district level equal the products supplied volumes as reported in the PSA, 1992 through 1996. For example, the 1996 survey yielded a volume for distillate residential use in PAD District II of 903,197 thousand gallons. The total distillate volume for PAD District II was $6,488,200$ from the survey, after subtracting the volume coming from alternate sources. The product supplied total distillate volume for PAD District II was $6,359,193$ after removing alternate source volume. Hence, the adjusted 1996 estimate of distillate for residential use in PAD District II was $(6,359,193 / 6,488,200) \times 903,197=885,239$.

For distillate fuel oil in Tables 13 through 24, the PAD district adjustments were made to each State and product-use category except on-highway diesel use, and electric utility use.

Volumes of residual fuel oil in Tables 13 through 24 were adjusted at the national level to equal the products supplied estimate. The 1996 survey yielded $13,256,998$ thousand gallons of residual fuel oil, while the products supplied volume at the national level was $13,041,042$ thousand gallons. Thus, the adjustment factor at the national level for 1996 was 1.02, after subtracting the volumes coming from alternate sources. For residual fuel oil, the adjustments were made to each State and end-use category except electric utility use. 


\section{Note 4: End-Use Descriptions and Petroleum Product Definitions}

\section{End-Use Descriptions}

Residential Use. Includes sales for use in private households for space heating, water heating, cooking, etc. Sales to farm houses are reported under "Farm Use."

Commercial Use. Includes sales for use in nonmanufacturing establishments such as motels, apartment buildings, restaurants, retail stores, institutions, government buildings, schools, and laundries for space heating, water heating, and cooking.

Industrial Use. Includes sales to mines, smelters, and plants engaged in producing manufactured products, processing and assembling goods, etc. Also includes space heating. Excludes oil company use, as defined below.

Oil Company Use. Includes sales to drilling companies, pipelines or other related oil companies not engaged in the selling of petroleum products. Includes fuel oil that was purchased or produced and used by company facilities for the operation of drilling equipment, other field or refinery operations, and space heating at petroleum refineries, pipeline companies, and oil-drilling companies. Oil used to bunker vessels is counted under vessel bunkering. Sales to other oil companies for field use are included, but sales for use as refinery charging stocks are excluded.

Farm Use. Includes sales for use in tractors, irrigation pumps and other agricultural machinery, as well as that used for crop drying, smudge pot fuel, space heating of buildings (including farm houses), cooking, and any other use by the agriculture industry.

Electric Utility Companies. Includes sales to electric utility companies, including that used for the generation of electricity. Volumes imported and used directly by electric utility companies are also included.

Railroad Use. Includes sales to railroads, for any use, including that used for heating buildings operated by railroads.

Vessel Bunkering. Includes sales for the fueling of commercial or private boats, such as pleasure craft, fishing boats, tugboats, and ocean-going vessels, including vessels operated by oil companies. Excluded are volumes sold to the U.S. Armed Forces.
On-Highway Diesel Use. Includes sales for use in motor vehicles. Volumes used by companies in the marketing and distribution of petroleum products are also included.

Military Use. Includes sales to the Armed Forces, including volumes sold to the Defense Fuel Supply Center (DFSC) for use by all branches of the Department of Defense (DOD).

Off-Highway Use. Includes sales for use in:

1. Construction. Construction equipment including earthmoving equipment, cranes, stationary generators, air compressors, etc.

2. Other. Sales for off-highway uses other than construction. Sales for logging are included in this category. Volumes for off-highway use by the agriculture industry are reported under "Farm Use."

All Other Uses. Sales for all other uses not included in any other end-use category are reported here.

\section{Definitions of Petroleum Products and Other Related Terms}

ASTM. The acronym for the American Society for Testing and Materials.

Distillate Fuel Oil. A general classification for one of the petroleum fractions produced in conventional distillation operations. It is used primarily for space heating, on- and off-highway diesel engine fuel (including raillroad engine fuel and fuel for agricultural machinery), and electric power generation. Included are products known as No. 1, No. 2, and No. 4 fuel oils and No. 1, No. 2, and No. 4 diesel fuels.

1. No. 1 Distillate. A petroleum distillate which meets the specifications for No. 1 heating or fuel oil as defined in ASTM D 396 and/or the specifications for No. 1 diesel fuel as defined in ASTM Specification D 975.

a. No. 1 Diesel Fuel. A volatile distillate fuel oil with a boiling range between 300 and 575 degrees Fahrenheit and used in highspeed diesel engines generally operated under wide variations in speed and load. Includes type C-B diesel fuel used for city buses and similar operations. Properties are defined in ASTM Specification D 975.

b. No. 1 Fuel Oil. A light distillate fuel oil intended for use in vaporizing pot-type 
burners. ASTM Specification D 396 specifies for this grade maximum distillation temperatures of 400 degrees Fahrenheit at the 10-percent point and 550 degrees Fahrenheit at the 90-percent point, and kinematic viscosities between 1.4 and 2.2 centistokes at 100 degrees Fahrenheit.

2. No. 2 Distillate. A petroleum distillate which meets the specifications for No. 2 heating oil or fuel oil as defined in ASTM D 396 and/or the specifications for No. 2 diesel fuel as defined in ASTM Specification D 975.

a. No. 2 Diesel Fuel. A gas oil type distillate of lower volatility with distillation temperatures at the 90-percent point between 540 and 640 degrees Fahrenheit for use in high speed diesel engines generally operated under uniform speed and load conditions. Includes Type R-R diesel fuel used for railroad locomotive engines, and Type T-T for diesel-engine trucks. Properties are defined in ASTM Specification D 975.

(1) No. 2 Diesel $\leq .05 \%$ Sulfur. The sulfur level does not exceed 0.05 percent by weight.

(2) No. 2 Diesel $>.05 \%$ Sulfur. The sulfur level is above 0.05 percent by weight.

b. No. 2 Fuel Oil. A distillate fuel oil for use in atomizing type burners for domestic heating or for moderate capacity commercialindustrial burner units. ASTM Specification $\mathrm{D} 396$ specifies for this grade distillation temperatures at the 90-percent point between 540 and 640 degrees Fahrenheit, and kinematic viscosities between 2.0 and 3.6 centistokes at 100 degrees Fahrenheit.

3. No. 4 Fuel. A fuel oil for commercial burner installations not equipped for preheating facilities. It is used extensively in industrial plants. This grade is a blend of distillate fuel oil and residual fuel oil stocks that conforms to ASTM Specification D 396 or Federal Specification VV-F-815C; its kinematic viscosity is between 5.8 and 26.4 centistokes at 100 degrees Fahrenheit. Also included is No. 4-D, a fuel oil for lowand medium-speed diesel engines that conforms to ASTM Specification D 975.

Kerosene. A petroleum distillate that boils at a temperature between 300 and 550 degrees Fahrenheit, that has a flash point higher than 100 degrees Fahrenheit by ASTM Method D 56, that has a gravity range from 40 to 46 degrees API, and that has a burning point in the range of 150 degrees to 175 degrees Fahrenheit. Included are the two classifications recognized by ASTM Specification D 3699: No. 1-K and No. 2-K, and all grades of kerosene called range or stove oil which have properties similar to No. 1 fuel oil, but with a gravity of about 43 degrees API and a maximum end-point of 625 degrees Fahrenheit. Kerosene is used in space heaters, cook stoves, and water heaters and is suitable for use as an illuminant when burned in wick lamps. NOTE: Respondents to the EIA-821 survey were instructed to report all volumes in accordance with what the product was sold as, regardless of the actual specifications of that product. For example, if a No. 2 distillate was sold as a heating oil or fuel oil, the volume would be reported in the category "No. 2 Fuel Oil" even if the product conformed to the higher specifications of a diesel fuel.

PADDistrict. Petroleum Administration for Defense Districts

PAD District I:

Subdistrict IA. Connecticut, Maine, Massachusetts, New Hampshire, Rhode Island, and Vermont

Subdistrict IB. Delaware, District of Columbia, Maryland, New Jersey, New York, and Pennsylvania

Subdistrict IC. Florida, Georgia, North Carolina, South Carolina, Virginia, and West Virginia

\section{PAD District II:}

Illinois, Indiana, Iowa, Kansas, Kentucky, Michigan, Minnesota, Missouri, Nebraska, North Dakota, South Dakota, Ohio, Oklahoma, Tennessee, and Wisconsin

\section{PAD District III:}

Alabama, Arkansas, Louisiana, Mississippi, New Mexico, and Texas

\section{PAD District IV:}

Colorado, Idaho, Montana, Utah, and Wyoming

\section{PAD District V:}

Alaska, Arizona, California, Hawaii, Nevada, Oregon, and Washington

Residual Fuel Oils. The topped crude of refinery operations, which includes No. 5 and No. 6 fuel oils, as defined in ASTM Specification D 396 and Federal Specification, VV-F-815C; Navy Special fuel oil as 
defined in Military Specification MIL-F-859E including Amendment 2 (NATO symbol F-77); and Bunker $\mathrm{C}$ fuel oil. Residual fuel oil is used for the production of electric power, space heating, vessel bunkering, and various industrial purposes.
United States. The 50 States and the District of Columbia.

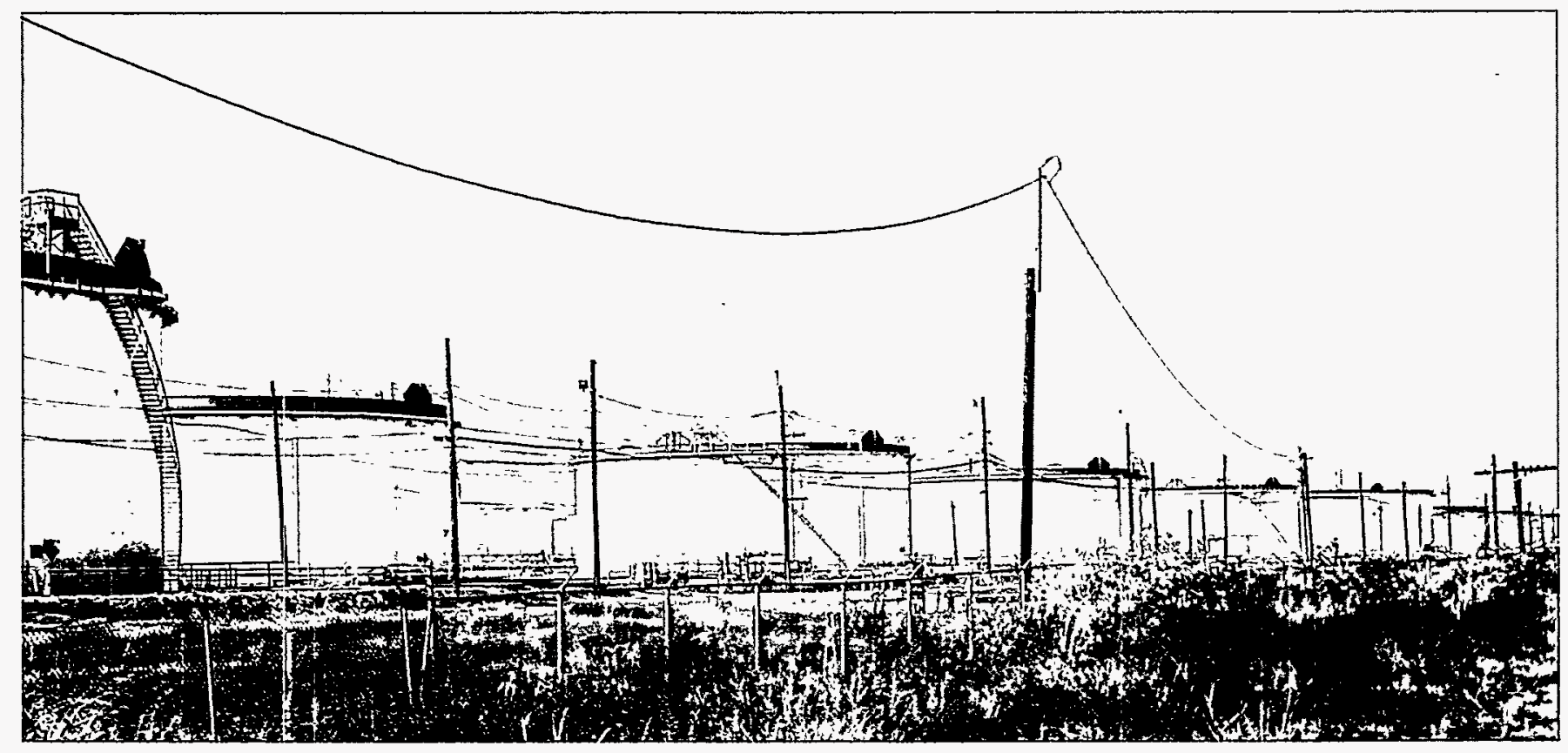

Storage and distribution of petroleum products are becoming more challenging in the 1990s due to a wide variety of new air quality regulations. 
Appendix B

\section{Form EIA-821: Annual Fuel Oil and Kerosene Sales Report}





\section{Appendix B}

EIA-821 (12-96)

Form Approved

U.S. DEPARTMENT OF ENERGY

OMB Number: 1905-0174

Energy Information Administration

Form Expires: $12 / 31 / 99$

\section{EIA-821: ANNUAL FUEL OIL AND KEROSENE SALES REPORT}

This report is mandatory under Public Law 93-275. For the provisions concerning the confidentiality of information and sanctions, see Sections V and VI of the instructions.

\section{PART I. IDENTIFICATION DATA}




EIA-821 (12-96)

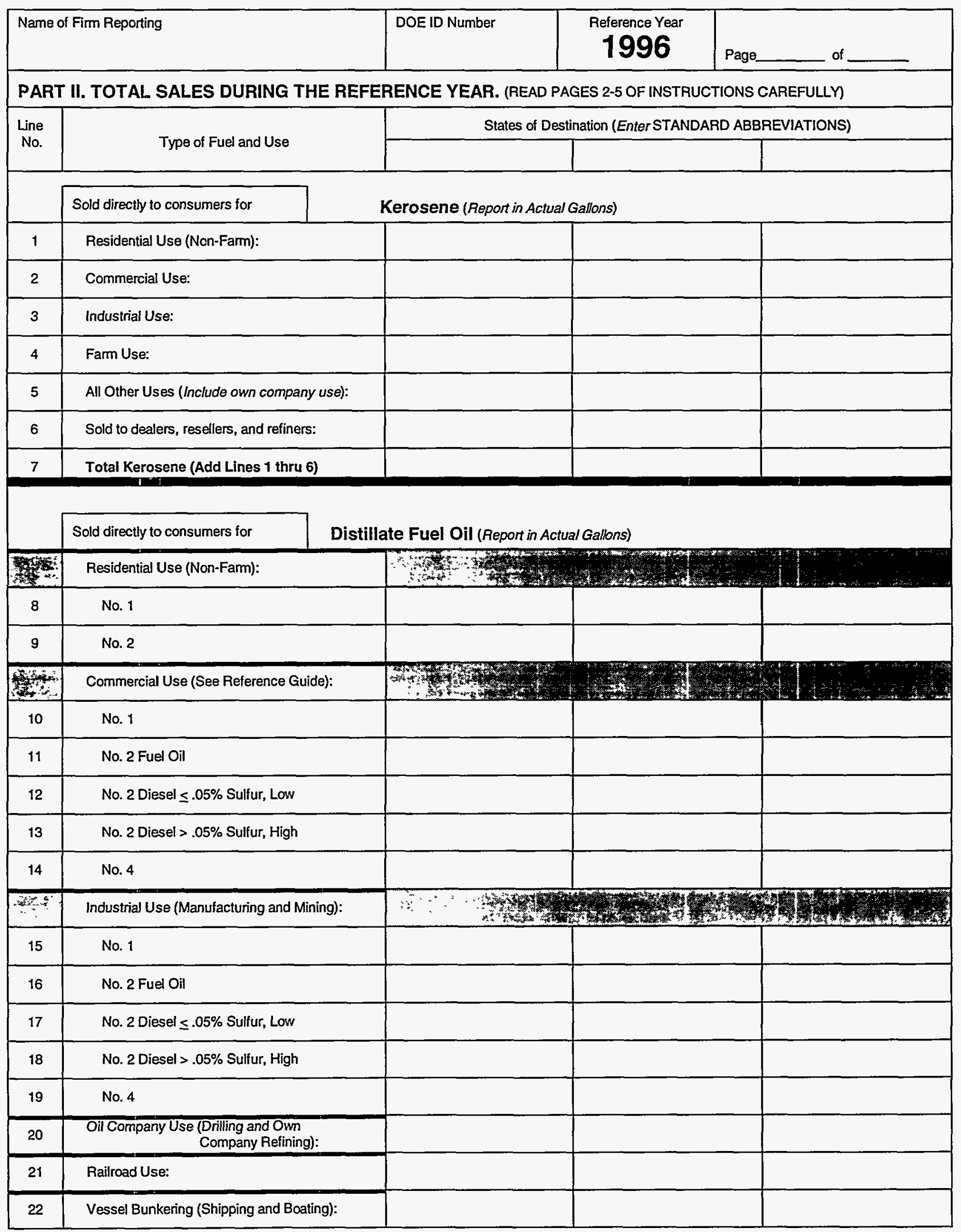




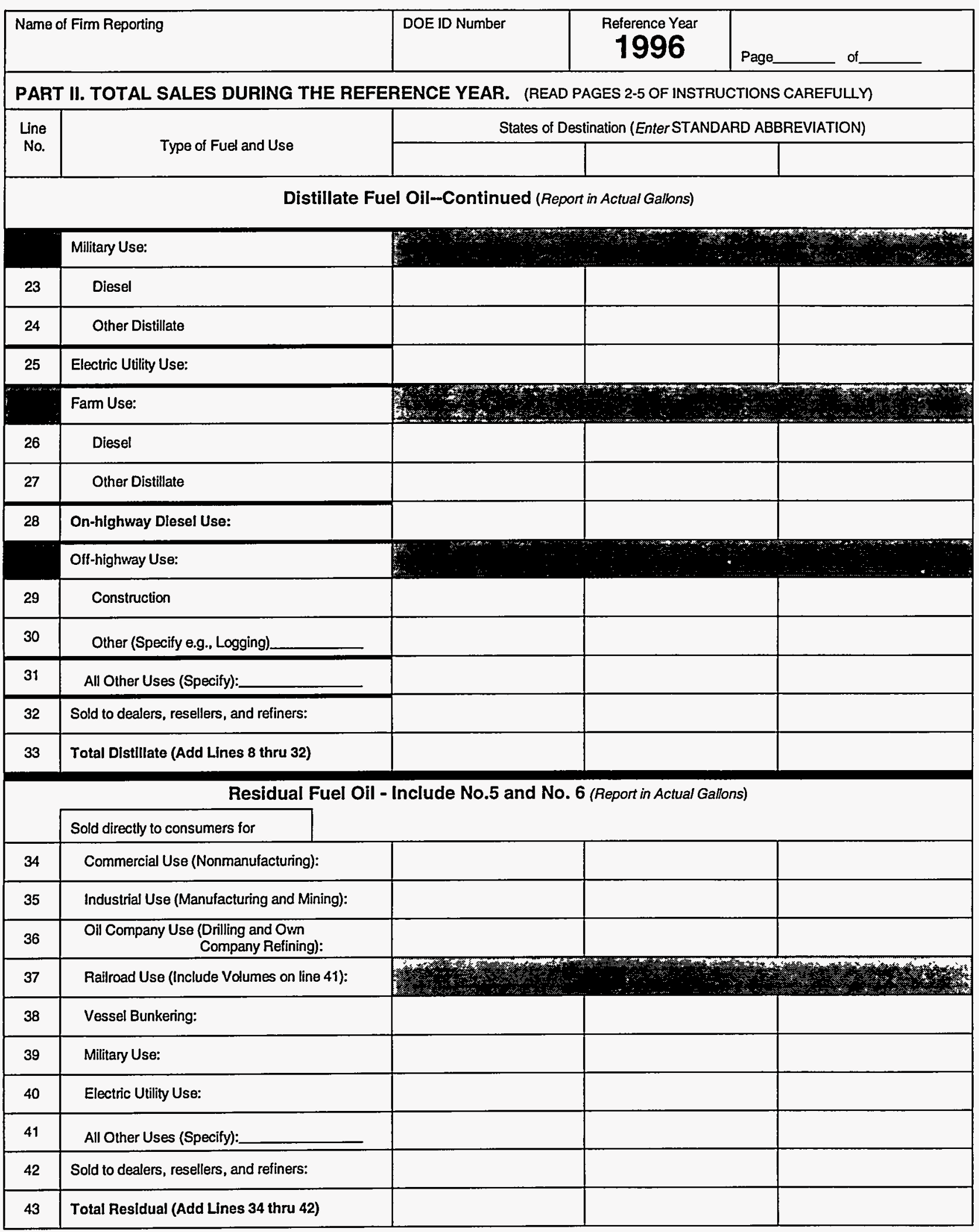




\section{U.S. Census Bureau Announces Census Catalog and Guide: 1997}

- Contents -

General and Reference

Agriculture Business

Communications

Construction

and Housing

Finance

Foreign Trade

Geography

Governments

Insurance

International

Manutacturing

and Mineral

Industries

Population

Real Estate

Transportation

Utilities

Sources of

Assistance
Helps you select from all the products

Every Census Bureau product issued mid-1993-1996:

Reports - CD-ROM - Computer tapes

Floppy disks - Internet access - Maps

AND

Many statistical publications from other Federal agencies

\section{Features facts about each product} Topics - Areas - Dates - Prices

\section{Makes finding the right product easy}

Title index - Detailed subject index

Guides to statistics from all censuses and surveys

\section{Identifies sources of assistance}

1,800 data center organizations

200 Census Bureau specialists 1,500 depository libraries

Other Federal statistical agencies

3 other directory lists

*8056

Superintendent of Documents Publications Order Form

YES, please send me the following:

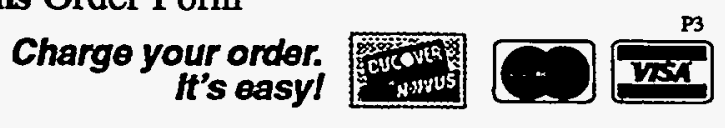

To phone your onders 202-512-1800 To fax your orders 202-512-2250

copies of Census Catalog and Guide: 1997 at $\$ 27$ (\$33.75 foreign), S/N 003-024-08824-0.

The total cost of my order is $\$$ Prices include regular domestic postage and handling and are subject to change.

\begin{tabular}{l}
\hline (Company or personal name) \\
\hline (Additional address/attention line) \\
\hline (Street address) \\
\hline (City, State, ZIP Code) \\
(Daytime phone including area code) \\
(Purchase Order No.)
\end{tabular}

Please Choose Method of Payment:

$\square$ Check payable to the Superintendent of Documents

$\square$ GPO deposit account

VISA or MasterCard account

\begin{tabular}{|l|l|l|l|l|l|l|l|l|l|l|l|l|l|l|l|l|l|}
\hline & & & & & & & & & & & & & & & & & \\
\hline
\end{tabular}

\begin{tabular}{l|l|l}
\hline & & \\
\hline
\end{tabular}

(Authorizing signature)

Mail To: Superintendent of Documents P.O. Box 371954, Pittsburgh, PA 15250-7954
Thank you for your order!

$6 / 97$ 